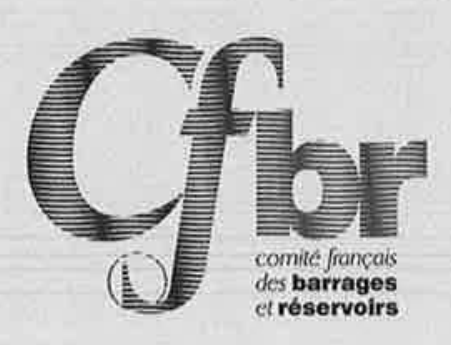

\title{
Recommandations
} pour la justification de la stabilité des barrages et des digues en remblai

\section{Recommandations provisoires}

Sous la direction de P. Royet et L. Peyras

P. Agresti, S. Aigouy, P. Anthiniac, B. Couturier, L. Deroo, L. Duchesne, E. Durand, J.-J. Fry, M. Galiana, D. Loudière, S. Mercklé, B. Touileb, C. Varon, E. Vuillermet 


\section{Avant-propos}

Ce document a été rédigé par un groupe de travail du Comité français des barrages et réservoirs (CFBR) qui a mené cette tâche sur la période de fin 2007 à début 2010. Son caractère, pour le moment provisoire, vise à rassembler les remarques concernant son application, en vue d'en faire un document définitif à l'échéance de deux ans.

\section{Liste des auteurs}

Stéphan AIgOuY (BETCGB)

Patrice ANTHINIAC (Coyne \& Bellier)

Bernard COUTURIER (Grenoble INP/ENSE3)

LUC DEROO (ISL)

Laurence DUCHESNE et Chiara CURZI (CNR)

Édouard DURAND (CETE Normandie-Centre)

Jean-Jacques FRY et Jean-Robert COURIVAUD (EDF-CIH)

Mathieu GALIANA (Cetmef)

Daniel LOUDIÈRE (CTPB)

Sébastien MERCKLÉ (Cemagref)

Caroline VARON (Safege)

Pierre AGRESTI, puis Bachir TOUILEB (Sogréah)

Éric VUILLERMET (BRL-i).

Paul Royet et Laurent Peyras (Cemagref) ont assuré la direction de ce projet.

Jean-Pierre Magnan (IFSTTAR) et Patrice Mériaux (Cemagref) ont fait une relecture minutieuse en phase finale du document. 


\section{Introduction}

\section{Pourquoi des recommandations pour la justification des ouvrages hydrauliques en remblai ?}

Le Comité français des barrages et des réservoirs a décidé de rédiger ces recommandations en partant du double constat que les pratiques françaises en matière de justification des ouvrages hydrauliques en remblai étaient hétérogènes et que les publications professionnelles disponibles présentaient également des différences sensibles entre elles. Le présent document propose donc d'harmoniser les pratiques sous forme de recommandations pour la justification de la stabilité des ouvrages hydrauliques en remblai en France.

Ces recommandations adoptent le format des méthodes semi-probabilistes aux états limites, à l'instar des Eurocodes ${ }^{1}$, qui constituent un référentiel standard bien adapté pour une harmonisation des pratiques. Cette présentation a l'avantage d'être utilisée dans de nombreux règlements du génie civil.

Ce document tire les bénéfices du travail précédent engagé par le CFBR et relatif aux barrages-poids. Le format des justifications est donc homogène à celui des recommandations françaises relatives aux barragespoids, conduisant au final à un ensemble cohérent de recommandations professionnelles.

\section{Les ouvrages hydrauliques en remblai et les Eurocodes}

Les Eurocodes structuraux (EN 1990 à 1999) sont un ensemble de normes européennes destinées à proposer un cadre commun pour la conception structurale des bâtiments et des ouvrages de génie civil, couvrant les aspects géotechniques, les situations sismiques, l'exécution et les structures provisoires. Ces normes ont progressivement été transposées en normes nationales : NF EN 1990 à NF EN 1999 pour la France.

Pour ce qui relève de l'application de ces textes au domaine des ouvrages hydrauliques en remblai, la norme NF EN 1990 ( Bases de calcul des structures ) indique dans son introduction (article 1.1, Domaine d'application) que, pour le calcul d'ouvrages spéciaux (par exemple installations nucléaires, barrages, etc.), d'autres dispositions que celles des EN 1990 à 1999 peuvent être nécessaires. De son côté, la norme NF EN 1997-1 « Eurocode 7 : Calcul géotechnique - Partie 1 : Règles générales » précise que les dispositions de la norme s'appliquent aux remblais de petits barrages et d'infrastructures (section 12, article 12.1), sachant que la notion de (c petits barrages » n'est pas définie dans l’Eurocode 7.

La justification des ouvrages de génie civil en remblai (en dehors des ouvrages hydrauliques) est réglementée par l'Eurocode 7 et son annexe nationale. Cette dernière précise que les approches de calcul qui s'appliquent sont les approches 2 et 3 , ce qui exclut l'utilisation de l'approche 1 en France. Frank et al. (2004) soulignent la difficulté de l'application de l'approche 2 pour la stabilité des remblais : au sein d'une même masse glissante, il existe une partie ayant un effet défa-

(1) Même si les Eurocodes ne s'appliquent pas directement aux grands barrages. vorable sur le glissement, et une partie ayant un effet favorable ; la limite entre ces deux parties étant différente pour chaque cercle de glissement testé, il devient donc délicat d'appliquer convenablement à ce stade les coefficients partiels sur les actions.

$\mathrm{Au}$ final, même si rien ne s'oppose aujourd'hui à utiliser les approches 2 ou 3 de l'Eurocode 7 (et en particulier l'approche 3) pour la justification des petits ouvrages hydrauliques en remblai, les Eurocodes ne constituent pas un cadre intégré de justification pour les ouvrages hydrauliques en remblai et l'ingénierie a recours aux recommandations professionnelles spécialisées. Par ailleurs, la cohérence entre l'Eurocode 7 et les recommandations relatives aux ouvrages hydrauliques, en particulier en ce qui concerne les niveaux de sécurité, n'est pas connue.

C'est ce contexte qui a conduit le CFBR à mettre en place un groupe de travail pour traiter de la justification des barrages et des digues en remblai.

\section{Domaine d'application des recommandations}

Ces recommandations s'appliquent à la justification de la stabilité des ouvrages hydrauliques en remblai dans le contexte français. Ces ouvrages comprennent: - les barrages en remblai de toutes hauteurs et de toute sorte : homogène, pseudo-homogène, zoné, à noyau central, à étanchéité amont, en enrochements, etc. ;

- les digues de toute sorte : fluviales, de canaux, de protection contre les inondations, etc.

Les ouvrages et organes suivants peuvent nécessiter des justifications particulières qui ne sont pas traitées dans le présent document:

- les barrages de stériles miniers ;

- les digues de protection contre les submersions marines;

- les batardeaux provisoires;

- les remblais d'ouvrages hydrauliques en terre armée ou renforcés avec des géotextiles;

- les masques de barrages en béton, béton bitumineux ou les dispositifs d'étanchéité par géomembrane.

Ce document est destiné à être utilisé dans le cadre de la justification d'ouvrages neufs et du diagnostic et du confortement d'ouvrages en service. Pour les ouvrages en service, on tient compte de l'historique de l'ouvrage et des données disponibles : données de chantier, résultats de l'auscultation, essais, etc.

\section{Démarche générale de la justification de la stabilité des ouvrages hydrauliques en remblai}

Dans ces recommandations, on examine successivement : les situations de projet, les actions, les résistances, les états limites et leurs conditions. Au final, le canevas des justifications est analogue aux pratiques actuelles, mais apparaît plus structuré et formalisé.

- Les situations de projet (partie 1) sont classées en trois catégories différenciées par l'intervalle de temps pendant lequel les distributions de toutes les données (actions, résistances) sont considérées comme constantes : 
- les situations normales d'exploitation. Elles se réfèrent aux conditions de d'exploitation normale de l'ouvrage ;

- les situations transitoires ou rares. Elles se réfèrent à des conditions temporaires de fonctionnement ou à des probabilités d'occurrence assez élevées sur la durée de vie de l'ouvrage ;

- les situations accidentelles (ou extrêmes - par référence aux publications de la CIGB). Elles se réfèrent à des conditions extrêmes applicables à l'ouvrage ou à des probabilités d'occurrence faibles sur la durée de vie de l'ouvrage.

Pour les ouvrages hydrauliques en remblai, il est apparu nécessaire d'introduire une catégorie spécifique de situations de projet liée aux crues que l'ouvrage est susceptible de subir durant sa vie : les situations de crue. On y distingue trois catégories : les situations rares de crue (dédiées à la justification des barrages écrêteurs de crue et des digues de protection contre les inondations), les situations exceptionnelles de crue (correspondant à la pratique des ( plus hautes eaux ), $\mathrm{PHE}$ ) et les situations extrêmes de crue (au-delà de laquelle l'intégrité de l'ouvrage ne serait plus assurée).

- Les actions sont réparties en trois catégories :

- les actions permanentes (partie 1) : essentiellement le poids propre de la structure ;

- les actions variables : les actions de l'eau qui, vu leur importance, constituent une partie spécifique du document (partie 3 traitant des modèles hydrauliques) ;

- les actions accidentelles : en particulier l'action du séisme d'évaluation de sécurité (SES).

Les actions permanentes sont prises en compte dans les calculs à partir de leur valeur caractéristique : celle-ci correspond à une estimation prudente de l'intensité de l'action et intègre donc la marge de sécurité sur l'intensité des actions permanentes. Pour les actions variables (les actions de l'eau), les valeurs représentatives sont choisies directement dans les différentes situations de projet en examinant les niveaux de remplissage de la retenue et les intensités qui en résultent. La modélisation hydraulique vise à fournir le champ des pressions interstitielles et le champ des gradients hydrauliques au sein de l'ouvrage en fonction des conditions aux limites hydrauliques appliquées. Enfin, l'action accidentelle sismique est définie en fonction du séisme d'évaluation de sécurité - SES. Notons que l'aléa sismique n'est pas traité dans ces recommandations, car il fait actuellement l'objet d'un travail spécifique conduit au sein d'un groupe de travail ad hoc (GT ( Séismes et barrages )) ; le cas échéant, il sera donc nécessaire de consulter le rapport à paraître.

- La formalisation des données géologiques et géotechniques en vue de la justification d'un ouvrage hydraulique en remblai comprend les trois étapes suivantes (partie 2) :

- le modèle géologique de la fondation vise à fournir des informations sur le niveau de fondation, sa capacité portante, son étanchéité ainsi que d'apprécier la stabilité des appuis de l'ouvrage, les risques de tassements différentiels et d'érosion de la fondation ;

- le modèle géométrique dresse ensuite une représentation simplifiée de la géométrie de l'ouvrage et de sa fondation ;
- le modèle géotechnique de la fondation et du remblai définit un cadre de représentation des propriétés hydrauliques et mécaniques des fondations et de l'ouvrage.

- Les propriétés de résistance des matériaux sont prises en compte dans les calculs à partir de leur valeur caractéristique (partie 2) : celle-ci correspond à une estimation prudente de la valeur de la résistance du matériau et intègre donc une partie de la sécurité sur les valeurs des résistances. Dans les Eurocodes, cette prudence dans l'estimation des paramètres est prise en compte par un fractile à $95 \%$ (ou $5 \%$ selon le caractère favorable ou défavorable) de la loi de distribution de la résistance considérée. La valeur caractéristique d'une propriété correspond donc à une valeur ayant une fréquence donnée de ne pas être atteinte lors d'une hypothétique série illimitée d'essais. L'évaluation des résultats d'essais par des méthodes statistiques ne peut être réalisée que lorsque les données proviennent de populations identifiées suffisamment homogènes et qu'un nombre suffisant d'observations est disponible. Toutefois, l'utilisation des statistiques n'est que rarement possible et n'est pas toujours pertinente. L'estimation prudente doit alors faire appel au jugement de l'expert, à partir des résultats d'essais disponibles ou à partir de valeurs guides issues de la littérature. La valeur caractéristique correspond ainsi à une estimation experte prudente de la valeur de l'action ou de la résistance du matériau, responsable de l'apparition des états limites.

- On justifie la stabilité des barrages en remblai pour différents états limites, c'est-à-dire pour différents phénomènes préjudiciables contre lesquels on cherche à se prémunir. Les recommandations traitent des états limites de glissement et de stabilité d'ensemble, des états limites de déformation (tassement et défaut de portance) et de l'état limite de soulèvement hydraulique du pied aval (partie 4). Pour les digues, on ajoute aux états limites précédents les états limites d'affouillement et d'érosion des berges (parties 5 et 6).

Certains états limites n'ont pas été traités dans ces recommandations, dans l'attente des résultats relatifs à des travaux en cours de réalisation:

- l'état limite de stabilité en situation sismique : le ministère chargé de l'écologie a engagé un travail de synthèse sur la question des méthodes de justification de la stabilité au séisme des ouvrages hydrauliques;

- les états limites de résistance à l'érosion interne et à la surverse : le projet de recherche ERINOH (ERosion INterne des Ouvrages Hydrauliques) a conduit à la mise en chantier d'un guide technique destiné en particulier à proposer des critères de justification de ces états limites.

- Pour chaque état limite, on écrit la condition d'état limite (parties 4, 5 et 6), qui fait intervenir :

- les actions, prises en compte au moyen des valeurs caractéristiques pour les actions permanentes et au moyen des valeurs représentatives correspondant aux situations de projet pour les actions de l'eau ;

- les propriétés de résistances, prises en compte au moyen des valeurs caractéristiques. Chaque valeur caractéristique est pondérée par un coefficient partiel 
- noté $\gamma_{m}$ - prenant en charge l'incertitude pesant sur la connaissance de la propriété. Le jeu de coefficients partiels adopté a été inspiré par l'approche 3 de l'Eurocode 7, modulé par la pratique de la justification des ouvrages hydrauliques qui introduit une différentiation des coefficients partiels selon la situation de projet;

- le coefficient de modèle, s'appliquant de façon conventionnelle du côté du terme moteur de la condition d'état limite. Le coefficient de modèle - noté $\gamma_{d}-$ prend en charge toutes les incertitudes ne relevant pas de la connaissance des propriétés de résistance, en particulier les incertitudes relatives au modèle hydraulique et au modèle d'état limite. En pratique, les coefficients de modèle ont été obtenus par calibration conventionnelle, le principe consistant à rechercher la meilleure équivalence entre les niveaux de sécurité de la méthode semi-probabiliste proposée et ceux résultant des pratiques traditionnelles déterministes, de manière à s'éloigner le moins possible - en moyenne des dimensionnements classiques. Ainsi, le coefficient de modèle joue le rôle d'ajustement entre les critères déterministe et semi-probabiliste.

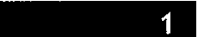

\section{Bases de calculs}

\section{1.}

\section{Situations de projet}

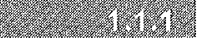 \\ Généralités}

Les situations de projet modélisent un ensemble de conditions physiques représentant les conditions réelles auxquelles l'ouvrage est soumis et qui se produisent au cours d'une certaine durée pendant laquelle les distributions de toutes les données (actions, résistances) sont considérées comme constantes. Elles correspondent à des chargements dans lesquels l'ouvrage est susceptible de se trouver durant sa vie : cote normale d'exploitation, crue de projet, séisme, retenue vide, défaillance particulière d'un composant de l'ouvrage, etc.

Le concepteur définit dans les notes de calculs les situations qu'il envisage pour le projet. Pour cela, il tient compte de la conception du projet, du mode de fonctionnement des installations, de l'environnement auquel l'ouvrage est soumis et de la vraisemblance des situations de défaillances.

Les conditions d'exploitation de l'ouvrage et les sollicitations hydrauliques associées permettent au concepteur de définir les situations de projet hydrauliques, qui revêtent une importance particulière pour les barrages et les digues en remblai.

Les situations de projet précisent les spécifications détaillées à prendre en compte dans les justifications et dans la conception:

- l'environnement réglementaire, social, économique, hydrogéologique, hydrologique dans lequel l'ouvrage s'inscrit, en particulier :
- le niveau de la rivière ou du fleuve pour les digues,

- les crues courantes, rares, exceptionnelles et extrêmes,

- les variations de niveau des eaux souterraines, y compris dans les versants,

- les conditions d'exploitation de l'ouvrage ;

- les actions :

- le poids propre et les surcharges dues au trafic,

- les actions de l'eau, en particulier les niveaux hydrostatiques de la retenue ou de la rivière et - les gradients hydrauliques en fondation et dans le remblai,

- l'action des séismes,

- etc. ;

- la description du terrain sur lequel l'ouvrage est construit :

- la disposition et la classification des différentes zones de sol ou de roches,

- le modèle géologique et géotechnique, en fondation et en remblai,

- les effets du temps ou de l'environnement sur les propriétés des matériaux,

- les propriétés géotechniques et leur valeur de calcul,

- les défaillances technologiques particulières envisagées pour l'ouvrage.

Les situations de projet de court terme et de long terme doivent être prises en compte.

Les situations de projet fixent les états limites à justifier, selon l'ouvrage considéré.

Les situations de projet à considérer doivent être sélectionnées en tenant compte des circonstances dans lesquelles l'ouvrage doit remplir sa fonction. Les situations de projet sont classées de la manière suivante :

- les situations normales d'exploitation. Elles se réfèrent aux conditions de d'exploitation normale de l'ouvrage ;

- les situations transitoires ou rares ${ }^{1}$. Elles se réfèrent à des conditions temporaires de fonctionnement ou à des probabilités d'occurrence assez élevées sur la durée de vie de l'ouvrage ;

- les situations accidentelles ou extrêmes. Elles se réfèrent à des conditions extrêmes applicables à l'ouvrage ou à des probabilités d'occurrence faibles sur la durée de vie de l'ouvrage.

Pour les ouvrages hydrauliques en remblai, il est apparu nécessaire d'introduire une catégorie spécifique de situations de projet liée aux crues que l'ouvrage est susceptible de subir durant sa vie : les situations de crue.

\footnotetext{
(t) On propose la nuance suivante entre transitoire et rare. Une situation transitoire est une situation de courte durée (au regard de la durée de vie de l'ouvrage) dont la survenue est quasiment certaine pendant la vie de l'ouvrage (fin de construction, vidange). Une situation rare est une situation dont la probabilité d'occurrence est assez élevée (sans pour autant être égale ou proche de 1) pendant la vie de l'ouvrage.
} 
Le concepteur répartit les situations de projet envisagées pour l'ouvrage dans les catégories suivantes (§ 1.1.2 à § 1.1.6), selon les conditions d'apparition ou leur probabilité d'occurrence sur la durée de vie de l'ouvrage. Cela concerne aussi bien :

- les situations liées à l'exploitation de l'ouvrage ;

- les situations traduisant l'occurrence d'un aléa naturel : crue, séisme, glissement de terrain, avalanche, etc. ;

- les situations de défaillance technologique d'un composant ou d'un élément de l'ouvrage.

\section{$4=15$}

\section{Situations normales d'exploitation}

Pour les situations normales d'exploitation, les conditions de stabilité doivent être assurées avec une marge de sécurité importante. Les hypothèses suivantes sont généralement adoptées :

- résistances des matériaux : résistances effectives correspondant aux paramètres à long terme ;

- poids propre des matériaux du remblai, de la fondation et des zones en déblai : poids volumique correspondant aux hypothèses imposées par le champ de pression interstitielle induit par le niveau d'eau à son niveau normal d'exploitation ;

- sollicitations hydrauliques : cote normale d'exploitation pour les barrages et les canaux et niveau moyen de la rivière pour les levées fluviales, avec le champ de pression interstitielle dans l'ouvrage obtenu pour cette cote (régime permanent établi).

Les principales situations normales d'exploitation considérées sont données ci-après et servent de guide pour le concepteur :

- Situation normale d'exploitation 1 : pour les barrages en eau ou les digues à charge permanente

La situation normale d'exploitation correspond à la cote atteinte dans la retenue ou dans le canal pour la cote normale d'exploitation. Cette situation de projet couvre les niveaux hydrauliques autorisés pour l'ouvrage en exploitation courante:

- pour les barrages, la situation normale d'exploitation comprend les niveaux hydrostatiques compris entre le niveau minimum autorisé d'exploitation et le niveau normal d'exploitation autorisé (cote RN, retenue normale) ;

- pour les digues en charge en permanence, le niveau normal d'exploitation (NNE) ou le niveau normal de navigation (NNN).

- Situation normale d'exploitation 2 : pour les barrages écrêteurs de crues ou les digues de protection contre les inondations

On prend généralement en compte la retenue vide pour les barrages écrêteurs et le niveau moyen du cours d'eau hors crue pour les digues de protection contre les inondations.

- Situation normale d'exploitation 3 : les barrages à marnage fort et fréquent

Pour les barrages dont la retenue est vidangée ou remplie très fréquemment (à l'instar de certains bar- rages hydroélectriques remplis la nuit et vidés le jour ou de certains barrages d'altitude pour la production de neige de culture), il est loisible de considérer deux situations normales d'exploitation : i) la retenue pleine ; ii) la retenue en vidange rapide.

\section{-}

\section{Situations transitoires ou rares}

Pour les situations transitoires et rares, les conditions de stabilité doivent être assurées avec une marge de sécurité importante.

Les principales situations transitoires ou rares considérées sont données ci-après et servent de guide pour le concepteur.

\section{- Situation transitoire en cours et en fin de construc- tion \\ Cette situation de projet correspond généralement} à la fin de la mise en place des remblais, les pressions interstitielles de construction n'étant pas entièrement dissipées, en particulier si le matériau est peu perméable.

Mais pour certains ouvrages, des situations transitoires peuvent être plus critiques que la fin de la construction. C'est le cas d'ouvrages dont le remplissage commence avant la fin de la construction. Cette situation transitoire peut aussi intéresser les ouvrages construits sur des fondations argileuses ou peu perméables (susceptibles de développer des pressions interstitielles de construction) ou les remblais argileux ou peu perméables. Il s'agit d'apprécier les conditions de stabilité du remblai et de la fondation à court terme, à la fin de la construction du remblai (avant remplissage de la retenue) et avant la consolidation des matériaux.

- Situation transitoire de vidange rapide du réservoir ou de baisse rapide du niveau de la rivière

Cette situation de projet intéresse les vidanges, les décrues ou les baisses rapides des charges hydrauliques. Elle est considérée généralement pour les remblais constitués de matériaux peu drainants et est particulièrement contraignante pour les remblais en arciles étanches. Elle vise à vérifier les conditions de stabilité du talus amont (côté rivière) en condition saturée. La vitesse de vidange est évaluée en fonction de la capacité maximale des organes de vidange du barrage ou, dans le cas des digues, en fonction de la dynamique des crues du cours d'eau.

- Situation rare sismique SBE (séisme de base d'exploitation)

Dans les zones où la sismicité est forte, on considère un séisme de base d'exploitation (SBE). Au cours et après le SBE, l'ouvrage doit conserver un état de fonctionnement satisfaisant.

\section{(1)}

\section{Les situations de crue}

Trois situations de projet liées aux crues que l'ouvrage est susceptible de subir durant sa vie sont à considérer. 


\section{- Situation rare de crue}

Cette situation de projet concerne essentiellement les barrages écrêteurs de crues et les digues de protection contre les inondations. Le projeteur peut, dans certains cas, être amené à considérer une situation rare de crue pour les barrages de stockage.

Pour un barrage écrêteur de crues, on évalue le débit maximal non dommageable du cours d'eau en aval du barrage (par exemple débit de plein bord du lit mineur ou débit d'un ouvrage aval créant section de contrôle) et on dimensionne les pertuis pour délivrer au maximum ce débit. Si le débit entrant dans la retenue lui est supérieur, il y a stockage temporaire dans la tranche d'eau de laminage, jusqu'à atteindre la cote d'un déversoir de surface dont la fonction est d'assurer la sécurité du barrage lors des événements extrêmes.

Pour les digues de protection contre les inondations, la digue protège la plaine de l'inondation pour les crues courantes, mais pour les événements rares (jusqu'aux événements exceptionnels), c'est la rupture de la digue par surverse dont on souhaite se prémunir. A cette fin, la digue est équipée d'un déversoir ou aménagée pour être rendue déversante sur une partie de sa longueur. Lorsque cette cote de déversement est atteinte, l'inondation de la plaine va se faire de façon maîtrisée ; la montée lente du niveau de l'eau dans la plaine va ensuite limiter les conséquences d'une éventuelle rupture brutale de la digue par surverse.

La situation rare de crue correspond donc à un niveau de remplissage atteignant la cote de protection, c'est-à-dire :

- la cote du déversoir de surface d'un barrage écrêteur de crues :

- la cote du déversoir de sécurité d'une digue ou cote de la partie de la digue aménagée pour résister à la surverse (cote le cas échéant variable de l'amont à l'aval).

La cote de protection est associée à une crue ou à une famille de crues qui, vu la présence de l'ouvrage, ne seront pas dommageables pour les enjeux en aval du barrage ou dans le val protégé par la digue.

Aussi bien pour les barrages écrêteurs que pour les digues, la probabilité annuelle d'occurrence associée à cette famille de crues est habituellement de $10^{-1}$ à $10^{-2}$ selon les enjeux à protéger, soit une période de retour de 10 à 100 ans.

Pour certains barrages de stockage, le projeteur peut être amené à considérer une situation rare de crue dont la probabilité d'occurrence associée serait de l'ordre de $10^{-2}$, soit une période de retour d'environ 100 ans.

\section{- Situation exceptionnelle de crue}

Cette situation de projet concerne tous les barrages et elle peut être également prise en considération pour la justification des digues de protection contre les inondations.

Pour les barrages ou les canaux, elle correspond à la cote des plus hautes eaux (PHE), cote qui est normalement reprise dans l'arrêté d'autorisation ou le décret de concession de l'ouvrage et qui prend ainsi une valeur réglementaire. Cette situation de projet est obtenue en considérant classiquement la crue de projet associée à une période de retour qui dépend du niveau de sécurité exigé. On calcule la cote maximale atteinte par le plan d'eau en fonction des capacités de l'évacuateur de crues, tout en tenant compte éventuellement de l'écrêtement des hydrogrammes.

Pour les digues de protection contre les inondations à construire, ayant défini la période de retour en fonction du niveau de sécurité exigé, on calcule la ligne d'eau associée à la (aux) crue(s) correspondante(s) en tenant compte du fonctionnement du déversoir. Puis on calcule la revanche nécessaire pour se protéger des vagues et/ou des surcotes dans les courbes et on calcule ainsi le profil en long de la crête de la digue. Inversement, pour les digues existantes, à partir de la connaissance du profil en long de la crête et d'un calcul de revanche, on détermine un profil de ligne d'eau admissible vis-à-vis de considérations liées à la sécurité hydraulique de la digue. A ce profil peut être associée une période de retour par comparaison avec les lignes d'eau dans la rivière en crues, période de retour dont on vérifiera si elle satisfait les exigences de sécurité mentionnées plus haut.

La situation exceptionnelle de crue conduisant à la cote des PHE peut résulter de divers événements hydrologiques, depuis la crue courte présentant un débit de pointe très élevé (donc bien écrêtée par la retenue) jusqu'à la crue longue présentant un débit durablement élevé (écrêtement réduit) en passant par les crues à pointes multiples (écrêtement de la première pointe, mais pas des suivantes).

La situation exceptionnelle de crue correspond à un niveau de remplissage laissant encore une revanche pour se protéger de l'effet des vagues et des irrégularités de la ligne d'eau. Pour cette situation de crue, l'ouvrage doit répondre à tous les standards de sécurité, que ce soit sur le plan structural (résistance au cisaillement ou au glissement, résistance en fondation, résistance à l'érosion interne, résistance à l'affouillement) ou sur le plan hydraulique (pas de débordement des coursiers, ouvrages de dissipation correctement dimensionnés). On dispose donc encore de marges avant d'atteindre des états-limite de rupture.

La probabilité annuelle associée à cette situation de projet est définie dans les Recommandations pour le dimensionnement des évacuateurs de crues, à paraître prochainement sous l'égide du CFBR.

\section{- Situation extrême de crue}

Cette situation de projet concerne les barrages et les digues. Elle correspond à la cote au-delà de laquelle l'ouvrage risque de subir des dégâts majeurs par érosion du noyau ou du remblai, pouvant conduire rapidement à la rupture. L'atteinte de cette cote de danger conduit à un état limite ultime par surverse et/ou érosion.

Cette cote correspondant à la situation extrême de crue peut être déterminée de la façon suivante (pour un ouvrage en remblai) :

- la cote de la crête pour un remblai semi-homogène ou à masque amont ;

- la cote d'arase du noyau pour un remblai zoné ;

- la cote conduisant au débordement d'un coursier avec érosion du talus aval du remblai ;

- ou cote à justifier en cas d'ouvrages particuliers. 
La situation extrême de crue conduisant à la cote de danger peut résulter d'une famille de crues, avec différentes formes d'hydrogrammes.

La probabilité annuelle associée à cette situation de projet est définie dans les Recommandations pour le dimensionnement des évacuateurs de crues, à paraître prochainement sous l'égide du CFBR.

\section{2.}

\section{Les situations accidentelles}

Pour les situations accidentelles, les conditions de stabilité doivent être assurées avec une sécurité minimale.

Les situations accidentelles peuvent être liées à l'occurrence d'aléas extérieurs (hors aléa hydrologique traité dans les situations de crue). Dans cette catégorie, la principale situation accidentelle est la situation accidentelle sismique.

\section{- Situation accidentelle sismique}

Il s'agit de vérifier les conditions de stabilité de l'ouvrage sous l'action du séisme de projet. La sismicité en France métropolitaine étant faible ou modérée, on étudie une unique situation sismique accidentelle pour la justification des ouvrages hydrauliques. Elle correspond au séisme d'évaluation de sécurité. Pour ce séisme, la stabilité de l'ouvrage doit être assurée et l'ouvrage ne doit pas connaître de dommages susceptibles de remettre en cause sa sécurité.

Dans la situation accidentelle sismique, on adopte généralement, pour la sollicitation hydraulique (niveau d'eau et sous-pressions) et les paramètres géotechniques, des hypothèses analogues à celles de la situation normale d'exploitation.

- D'autres situations accidentelles liées à des aléas extérieurs peuvent, dans certains projets, être examinées et sont citées pour mémoire :

- situation accidentelle de grand glissement de terrain ou d'effondrement de versant ;

- situation accidentelle d'avalanche ;

- situation accidentelle de seiche provoquée par un séisme.

\section{1.}

\section{Autres situations de projet liées à la défaillance d'un composant de l'ouvrage}

Certaines défaillances d'éléments ou de composants impliqués directement dans la sécurité du barrage ou de la digue peuvent conduire à des situations transitoires ou rares ou à des situations accidentelles devant être spécifiquement étudiées. Parmi celles-ci, on peut citer :

- la défaillance d'une ou plusieurs vannes d'un évacuateur de crues ;

- la défaillance d'un évacuateur de surface par obstruction partielle ou totale du fait d'embâcles ;

- la défaillance du dispositif de rabattement des souspressions ;

- la défaillance du système de drainage, y compris le cas échéant d'une pompe d'évacuation;
- la défaillance d'une conduite traversante;

- la défaillance de l'étanchéité : noyau, masque amont, contact entre la galerie périmétrale et le masque amont ou la paroi moulée, défaut d'une paroi d'étanchéité (paroi moulée, rideau de palplanches...) ;

- le défaut d'un perré maçonné de protection d'une digue ;

- etc.

Ces défaillances peuvent amener à des niveaux hydrauliques potentiellement plus défavorables que les situations précédentes.

La détermination des situations rares ou accidentelles liées aux défaillances des éléments de sécurité de l'ouvrage relève d'études spécifiques d'analyse de risques, qui fixent au cas par cas les défaillances potentielles sur un ouvrage donné et les niveaux d'eau associés dans le réservoir ou la rivière. Pour les barrages de classes $A$ et $B$ et les digues de classes A, B et $C$, les études d'analyse de risques sont intégrées dans les études de dangers.

Les études d'analyse de risques vont permettre d'estimer les probabilités de défaillance de l'élément particulier analysé (dispositif de drainage, dispositif d'évacuation des crues...) combinée au niveau d'eau dans la retenue. On évalue ainsi une probabilité d'occurrence globale rattachée à un scénario combinant simultanément la défaillance d'un composant et un niveau d'eau.

Le niveau de détail et la précision de l'étude d'analyse de risques sont adaptés à la taille et aux enjeux de l'ouvrage. Pour une première approche, on pourra se contenter d'une estimation des probabilités de défaillance à dire d'expert ou à partir de la littérature, et on limitera le nombre de situations examinées à quelques combinaisons « défaillances/niveau de retenue » jugées a priori les plus caractéristiques ou les plus défavorables. Cette première approche permet de juger de l'opportunité d'études d'analyse de risques plus complètes.

En fonction de la probabilité ainsi évaluée, la situation examinée peut être considérée comme situation rare (probabilité supérieure à $10^{-3}$ à $10^{-4}$ par an) ou comme accidentelle (probabilité inférieure à $10^{-4}$ par an).

\section{I.}

\section{Récapitulatif des principales situations de projet}

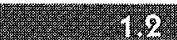

\section{Actions}

Les actions se répartissent en trois catégories :

- les actions permanentes qui sont le poids propre du remblai et les charges d'exploitation. Elles sont prises en compte dans les calculs à partir de leur valeur caractéristique : celle-ci correspond à une estimation prudente de l'intensité de l'action et intègre donc la sécurité sur l'intensité des actions permanentes;

- l'action variable de l'eau qui nécessite une approche spécifique, décrite en partie 3 (modèle hydraulique). 


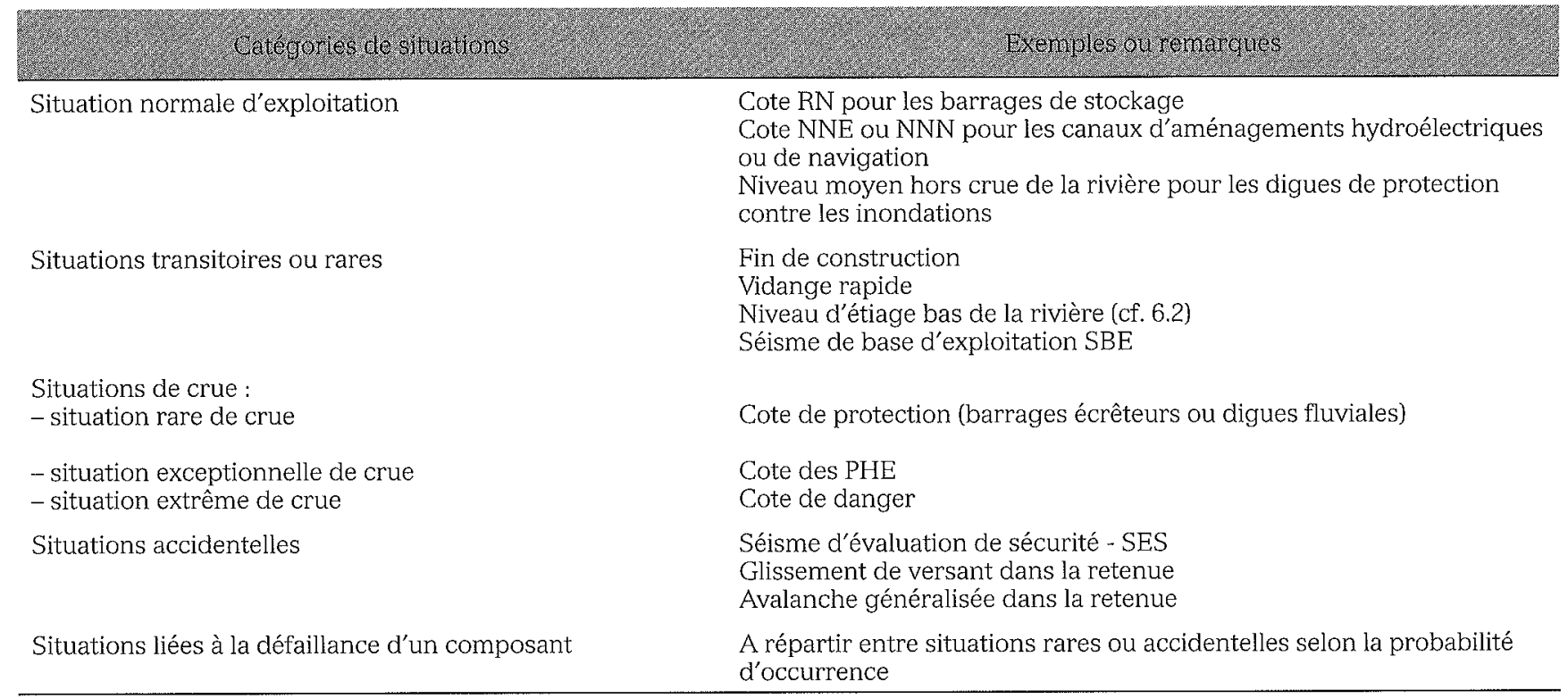

Cette action est évaluée dans les différentes situations de projet ;

- l'action accidentelle (ou rare) sismique qui est définie en fonction du (des) séisme(s) de projet et peut s'accompagner d'une évaluation spécifique de l'action de l'eau. L'action sismique n'est pas traitée dans le cadre du présent guide, cette dernière faisant l'objet d'un groupe de travail spécifique conduit sous l'égide du ministère chargé de l'écologie, et auquel il faudra se reporter.

Cet article ne traite donc que des actions permanentes.

Les actions permanentes sont les actions continues ou pratiquement continues dont l'intensité est constante ou très peu variable dans le temps. Les actions permanentes sont notées $G$.

Les intensités des actions permanentes sont obtenues à partir de leur valeur caractéristique. Une valeur caractéristique $G_{k}$ d'une action permanente $G$ est une estimation prudente de l'intensité de l'action.

La valeur caractéristique d'une action peut parfois être calculée de façon statistique lorsqu'on dispose d'essais suffisamment nombreux et lorsque la variabilité des paramètres rentrant dans le calcul de l'action permanente est limitée. Toutefois, l'utilisation des statistiques n'est que rarement possible. L'estimation prudente fait alors appel au jugement de l'expert, à partir des résultats d'essais disponibles ou à partir de valeurs guides issues de la littérature. La valeur caractéristique d'une action permanente correspond alors à une estimation experte prudente de la valeur de l'action.

Une action permanente $G$, en l'absence de données, peut être bornée par deux valeurs caractéristiques. En effet, selon l'état limite ou la situation de projet considéré, l'estimation ( prudente ) peut correspondre à une valeur supérieure $G_{k \text {-sup }}$ ou à une valeur inférieure $G_{k \text {-inf }}$
- Poids propre

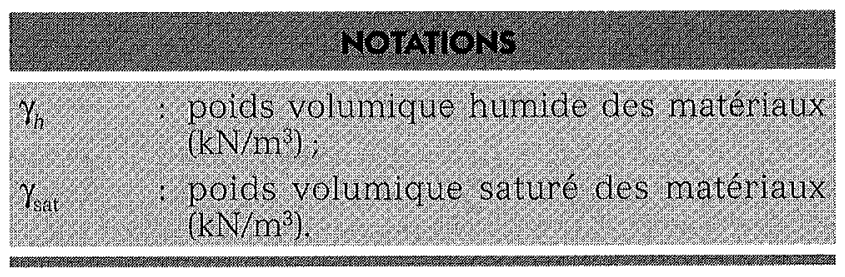

Principe de calcul : les actions des forces de gravité sont calculées directement à partir des poids volumiques des différents matériaux composant la fondation ou entrant dans le zonage du remblai.

On prend en compte le poids volumique humide $\gamma_{h}$ de chacun des matériaux. Pour les matériaux saturés, on considère donc $\gamma_{\text {sat }}$

Les forces de gravité pouvant avoir, selon les zones, un effet favorable ou défavorable sur la stabilité, l'estimation de la valeur caractéristique du poids volumique est une estimation par valeur inférieure dans le cas d'effet favorable et par valeur supérieure dans le cas d'effet défavorable.

Exemple, stabilité d'ensemble d'un remblai homogène avec une recharge aval :

- c'est $\gamma_{h \text {-sup }}$ que l'on prendra en compte pour le corps de remblai;

- c'est $\gamma_{\mathrm{h} \text {-inf }}$ que l'on prendra en compte pour la recharge aval.

Il convient, dans certains cas, de vérifier que les effets du tassement ne conduisent pas à une augmentation non négligeable du poids volumique tel que mesuré en cours de chantier.

\section{- Charges d'exploitation}

\section{Rotitions}

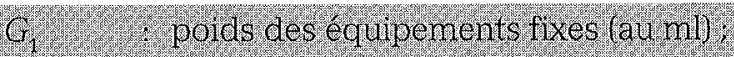
G. Harges variables dexploitation (au ni). 
Ces charges ne sont à considérer que si elles ont une intensité significative par rapport au poids propre du remblai. Le poids des équipements fixes est bien sûr une action permanente.

Quant aux charges liées au trafic sur le remblai, il est proposé de les prendre en compte également comme des actions permanentes, par souci de simplification, avec une valeur de $q=+10 \mathrm{kPa}$ appliquée sur toute la largeur de la crête.

Pour les digues, on peut être amené à adopter une intensité rare pour les charges d'exploitation correspondant à des travaux de confortement ou à des travaux d'urgence en crue ; on adopte alors une intensité rare de $\mathrm{q}=30$ à $80 \mathrm{kPa}$ selon les engins susceptibles d'être utilisés. Ce chargement relève alors de la catégorie des situations rares.

Toutefois, si ces actions étaient importantes relativement au poids propre du remblai et qu'elles étaient fréquemment appliquées, il conviendrait de les considérer comme des actions variables. On peut alors se référer aux intensités des actions dues au trafic proposées dans l'Eurocode 1 (norme NF EN 1991 " Actions sur les structures - Partie 2 : actions sur les ponts, dues au trafic $)$ ).

\section{-}

\section{États limites}

\section{- Définition d'un état limite}

Les états limites sont les phénomènes que l'on souhaite éviter. Ils correspondent à des états au-delà desquels l'ouvrage ne satisfait plus aux critères de dimensionnement. On distingue deux catégories d'états limites :

- les états limites de service (ELS), correspondant à des conditions au-delà desquelles les exigences d'aptitude au service de l'ouvrage ne sont plus satisfaites : les ELS sont les états limites associés au fonctionnement de la structure ou des éléments structuraux en utilisation normale d'exploitation;

- les états limites ultimes (ELU), associés à des pertes d'équilibres statiques ou des modes de ruine ; ils correspondent aux états limites qui concernent la sécurité des personnes et/ou la sécurité de la structure. projet.

Les états limites sont associés à des situations de

Dans la suite des recommandations, on s'intéresse prioritairement aux ELU pour lesquels on propose des critères de justification. On aborde également les ELS en en dressant une liste (pas forcément exhaustive) dans l'objectif de fournir un guide pour le projeteur. Les ELS devant être examinés de façon spécifique à chaque ouvrage, on ne fixe pas de critères d'état limite pour les ELS, tout au plus donne-t-on quelques recommandations.

\section{(1)}

\section{Modes de rupture (ELU)}

\section{- Définition du mot rupture}

La fonction principale d'un barrage ou d'une digue est de retenir l'eau. En conséquence, à l'instar de la CIGB, la perte de cette fonction principale est appelée rupture. Tout mouvement du remblai ou de ses ouvrages annexes, engendrant une incapacité à contenir la masse d'eau, est considéré comme une rupture. Nous l'étendons également à des ruptures en d'autres situations telles que la fin de construction.

\section{- Rupture par cisaillement}

Terzaghi rappelle que les particules de sol sont soumises à deux types de forces : les forces intergranulaires exercées par les grains voisins et les forces dues à l'eau présente dans le sol. Ainsi, le principe de Terzaghi écrit que la résultante des forces appliquées sur toute section de petite taille, comprenant au minimum une dizaine de grains, est équilibrée par la somme :

- du vecteur de contrainte effective intégrant l'ensemble des forces intergranulaires, transmises par les particules de sol ;

- et de la pression interstitielle, moyenne des forces dues à l'eau.

Le principe de Terzaghi est la clé de la rupture mécanique. Lorsque le vecteur de contrainte effective dépasse l'inclinaison du cône de frottement sur la section de petite taille, l'équilibre est rompu, il y a mouvement de cisaillement, la petite section devient une surface de glissement. La généralisation de cet état engendre le glissement du remblai. Il s'agit de la perte de stabilité mécanique par cisaillement. Il n'y a pas lieu de différencier la rupture par cisaillement statique et sismique, tant que les cycles provoqués par les trains d'onde sismiques ne génèrent pas de forte montée de la pression interstitielle.

Dans le cas extrême, un effort de traction peut s'ajouter au cisaillement. Si l'équilibre est rompu, le mouvement de cisaillement est accompagné d'une fissuration perpendiculaire à la contrainte effective de traction. Cet état apparaît lorsqu'un remblai en terre compacté est construit sur une fondation molle, les déformations de la fondation imposant des tractions à la base du remblai. Cet état est appelé perte de stabilité mécanique par défaut de portance de la fondation (ou poinçonnement). Ce cas particulier de la rupture par cisaillement ne peut être calculé par les méthodes des tranches, pour cette raison il doit être identifié et séparé du cas général.

Un autre cas extrême est généré lorsque la poussée de l'eau devient la composante principale de la force extérieure et qu'elle annule le poids des terres susjacentes. Perpendiculaire à la section où elle est appliquée, en augmentant, la poussée de l'eau dépasse le poids des couches sus-jacentes ou annule la contrainte effective, fracture le terrain et développe une fissuration verticale et/ou horizontale. Le sol se soulève ou perd sa consistance sous l'effet de l'eau qui le fissure. Cet état de rupture est appelé rupture par soulèvement hydraulique ou par annulation des contraintes effectives (boulance en statique et liquéfaction en dynamique).

\section{- Rupture par érosion}

Le principe de Terzaghi permet de traiter l'équilibre des forces macroscopiques au niveau d'une surface. En revanche, il ne permet pas de d'écrire l'équilibre d'un petit volume réduit jusqu'au grain seul. L'équilibre d'un 
grain nécessite la connaissance des forces physicochimiques, d'une part, et hydrodynamiques, d'autre part. L'expérience montre que les grains les plus fins ou ceux qui sont à la périphérie du sol sont sensibles aux forces hydrauliques, hydrostatiques et hydrodynamiques. La pression moyenne de l'eau ne suffit plus à décrire l'équilibre. Les variations de pression suscitées par le frottement de l'eau sur le grain ou par les fluctuations turbulentes de l'écoulement peuvent être du même ordre de grandeur que le poids déjaugé ou que la cohésion effective. Si la vitesse de l'écoulement augmente, alors le grain perd son l'équilibre d'abord par petits sauts, ensuite par vagues successives, pour finir emporté par le courant. Le mode de rupture est l'érosion.

Si l'écoulement est souterrain, on parle d'érosion interne. Si l'écoulement est à la surface de l'ouvrage, on parle d'érosion externe. Pour cette raison et dans l'état actuel de nos connaissances, il convient de distinguer les deux.

Dans le cas des digues proches d'un cours d'eau, on note que des mécanismes d'érosion (érosion régressive, érosion progressive, érosion de pied de berge (ou affouillement)) peuvent se coupler avec le mode de rupture par cisaillement (cf. § 2.2).

\section{- Part respective de chaque mode de rupture}

Il est possible d'avoir une idée précise de l'importance relative de chaque mode de rupture, en se référant aux statistiques de rupture des grands barrages (Foster, 2000). Le tableau suivant montre que l'érosion externe et l'érosion interne sont les dangers les plus forts, loin devant les problèmes de stabilité au glissement.

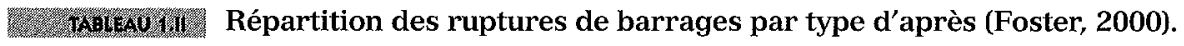

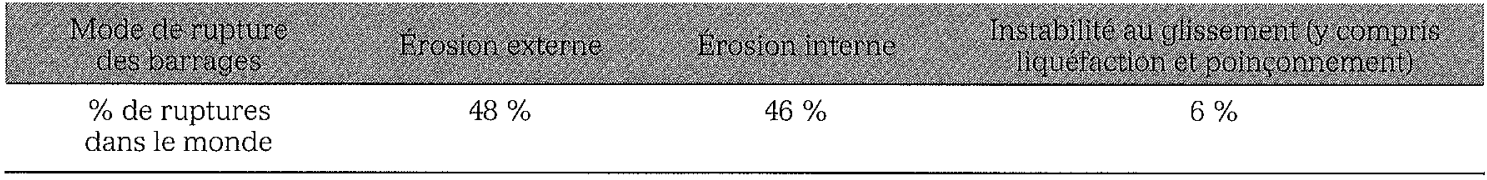

- Synthèse des ELU

A partir de l'analyse des modes de rupture, il est possible de présenter les principaux ELU. Le tableau suivant en fixe le découpage.

\section{8.}

\section{Les états limites de service (ELS)}

- Aptitude au service et fonctionnalités

L'aptitude au service d'un ouvrage hydraulique est liée à sa disponibilité durant sa durée de vie. Les principaux éléments structuraux de l'ouvrage, qui garantissent cette disponibilité, sont définis par des fonctionna- lités : l'étanchéité, la filltration, la stabilité, la protection, l'évacuation des crues, le suivi de la sécurité, la protection de l'environnement, etc. Quelques exemples de fonctions accomplies par les ouvrages hydrauliques dont la défaillance relève d'ELS sont donnés ci-après : - l'étanchéité est l'aptitude à retenir l'eau et à éviter des pertes excessives vis-à-vis de la sécurité et de l'économie du projet;

- la filtration est l'aptitude à retenir les particules emportées par l'eau. Pour les filtres au contact avec l'étanchéité, ils ont pour fonction de limiter les fuites en cas d'accident;

- le drainage est l'aptitude à évacuer les fuites, sans mise en charge des drains

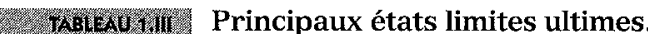

\begin{tabular}{|c|c|c|}
\hline \multirow{4}{*}{$\begin{array}{l}\text { Forces intergranulaires } \\
\text { et hydrostatiques à l'échelle globale }\end{array}$} & \multirow[t]{3}{*}{ Cisaillement } & Stabilité d'ensemble (glissement) \\
\hline & & $\begin{array}{l}\text { Stabilité sismique sans montée } \\
\text { de sous-pression }\end{array}$ \\
\hline & & $\begin{array}{l}\text { Défaut de capacité portante } \\
\text { (rupture par poinçonnement) }\end{array}$ \\
\hline & Soulèvement du sol & Soulèvement hydraulique \\
\hline \multirow{2}{*}{$\begin{array}{l}\text { Forces hydrodynamiques à l'échelle } \\
\text { globale }\end{array}$} & \multirow[t]{2}{*}{ Liquéfaction statique ou dynamique } & Boulance \\
\hline & & Liquéfaction \\
\hline \multirow{3}{*}{$\begin{array}{l}\text { Forces hydrodynamiques à l'échelle } \\
\text { locale }\end{array}$} & \multirow[t]{3}{*}{ Érosion } & Érosion interne \\
\hline & & Érosion de surverse \\
\hline & & Affouillement \\
\hline
\end{tabular}


- la protection est l'aptitude à protéger la surface du remblai des effets de l'environnement : agents atmosphériques et humains ;

- l'évacuation des crues est l'aptitude à évacuer les eaux en situation de crue rare à extrême ;

- la vidange est la capacité à abaisser partiellement ou totalement le niveau du réservoir ;

- le suivi de la sécurité est l'aptitude à repérer d'une part les effets du vieillissement avant qu'ils n'endommagent profondément l'ouvrage et d'autre part les conséquences des accidents.

\section{- Pertes d'exigence d'aptitude au service}

Les exigences d'aptitude au service propres à chaque fonctionnalité sont déclinées en une ou plusieurs conditions à satisfaire. Le tableau 1.IV indique des exemples de pertes de ces conditions (ELS) et de critères de vérification, à examiner et définir précisément au cas par cas pour chaque ouvrage particulier. On ne vise pas ici l'exhaustivité.

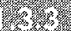

\section{Les scénarios de défaillance des ouvrages hydrauliques}

L'évaluation de la sécurité des ouvrages hydrauliques en remblai ne peut être réduite à la seule évaluation de la sécurité structurale, c'est-à-dire à la seule évaluation de l'occurrence des ELU. En effet, la défaillance structurale est la cause de pertes de fonctionnalité successives, et est donc liée à l'occurrence de différents ELS conduisant à la rupture (ELU). Ainsi, la notion de scénario de défaillance est fondamentale et le projeteur doit s'intéresser à toute la chaîne de défaillances fonctionnelles conduisant à la défaillance structurale de l'ouvrage.

Il existe des méthodes spécifiques pour l'analyse des scénarios de défaillance des ouvrages de génie civil, qui peuvent être appliquées aux ouvrages hydrauliques. Ces méthodes relèvent de la Sûreté de Fonctionnement. Une analyse des scénarios de défaillance d'un ouvrage comprend les trois principales étapes suivantes :

- l'analyse fonctionnelle : elle vise à définir précisément le système étudié, ses composants (l'analyse structurelle) et son environnement, et conduit aux différentes fonctionnalités que l'ouvrage doit accomplir (\$ 1.3.2). Il existe plusieurs méthodes d'analyse fonctionnelle dont certaines sont bien adaptées aux ouvrages du génie civil (Zwingelstein, 1996) ;

- l'analyse des défaillances : sur les bases de l'analyse fonctionnelle, elle vise à rechercher les modes de défaillance de l'ouvrage et de ses composants, dans des conditions de fonctionnement données (situations de projet). Il existe de nombreuses méthodes de la Sûreté de Fonctionnement pour l'analyse des défaillance : l'analyse préliminaire de dangers (APD), la méthode HAZOP, l'analyse des modes de défaillance, de leurs effets - de leur criticité - (AMDE/AMDEC) constituent des techniques bien adaptées aux systèmes mécaniques (Villemeur, 1988);

- les scénarios de défaillance : cette étape consiste à produire une modélisation des modes de fonctionnement (et de dysfonctionnement) du système et permet de représenter son comportement par des scénarios enchaînant les modes de défaillance. Il existe trois principales méthodes de modélisation des scénarios : la méthode des arbres d'événements (MAE), la méthode des arbres des causes (MAG) et la méthode du noud papillon.

A l'issue de l'analyse des scénarios de défaillance, l'étude quantitative des risques peut être engagée : elle comprend l'évaluation de la sécurité structurale de l'ouvrage, par exemple par une analyse semi-probabiliste comme cela est proposé dans ces recommandations, mais aussi, une analyse de l'occurrence des défaillances liées aux pertes de fonctionnalité. Au final, l'agrégation des différentes mesures d'occurrence des états limites (ELS et ELU) conduit à une évaluation de la sécurité (globale) de l'ouvrage.

L'étude des scénarios de défaillance des ouvrages hydrauliques, intégrant la démarche brièvement décrite dans cette section, est dorénavant prévue dans la règlementation française relative à la sécurité des ouvrages hydrauliques à travers les études de dangers (décret du 11 décembre 2007 et arrêté du 12 juin 2008). Les études de dangers s'imposent pour les barrages de classe $\mathrm{A}$ et $\mathrm{B}$ et les digues de classe $\mathrm{A}, \mathrm{B}$ et $\mathrm{C}$.

\section{1.x}

\section{Les différentes phases de vie des ouvrages hydrauliques}

Les états limites peuvent intervenir au cours des différentes phases de vie de l'ouvrage, la construction, l'exploitation et l'effacement:

1) construction : on note principalement des ruptures par surverse et par glissement (en cas de fondation argileuse ou de remblai argileux) ;

2) exploitation : l'érosion interne et la surverse sont les modes de rupture les plus fréquents ;

3) effacement : l'effacement d'un ouvrage hydraulique (notamment les barrages) est une notion qui a été appliquée récemment. Elle nécessitera quelques rares études spécifiques. En revanche, cette phase est essentielle pour les remblais de stérile.

La justification de la sécurité de l'ouvrage consistera à vérifier que la sécurité vis-à-vis d'un état limite est suffisante pour les différentes situations de projet dimensionnantes de chaque phase de vie étudiée.

\section{2}

\section{Données géologiques, géomorphologiques et géotechniques}

La démarche adoptée pour la formalisation des données géotechniques en vue de la justification d'un barrage ou d'une digue en remblai peut être synthétisée de la façon suivante

- établissement du modèle géologique de la fondation (§ 2.1) ;

- considérations sur la géomorphologie fluviale, principalement pour le cas des digues fluviales (\$ 2.2);

- établissement du modèle géométrique (\$2.3) ;

- établissement du modèle géotechnique de la fondation et du remblai : détermination des valeurs caractéristiques des paramètres géotechniques (§ 2.4);

- établissement du modèle hydraulique (chapitre 3) 


\begin{tabular}{|c|c|c|c|}
\hline $\begin{array}{l}\text { Étanchéité } \\
\text { Et1 }\end{array}$ & $\begin{array}{l}\text { Absence de fissuration suite au } \\
\text { glissement, aux tassements et } \\
\text { pérennité de l'étanchéité sous } \\
\text { chargement statique }\end{array}$ & $\begin{array}{l}\text { Par vieillissement ou accident, } \\
\text { les fuites sont supérieures au } \\
\text { débit de dimensionnement, } \\
\text { qu'elles soient inadmissibles } \\
\text { pour la sécurité (érosion } \\
\text { interne et stabilité) ou pour } \\
\text { l'économie du projet }\end{array}$ & $\begin{array}{l}\text { Évaluation des conditions } \\
\text { minimales d'étanchéité } \\
\text { assurant la fiabilité. Par } \\
\text { exemple, estimation de deux } \\
\text { seuils de débit de fuite: } \\
\text { 1. débit de dimensionnement, } \\
\text { 2. débit pouvant justifier } \\
\text { une intervention à cause des } \\
\text { conséquences économiques ou } \\
\text { sécuritaires }\end{array}$ \\
\hline
\end{tabular}

\section{Étanchéité et filtration} Et2

$\begin{array}{ll}\begin{array}{l}\text { Étanchéité } \\ \text { Et3 }\end{array} & \begin{array}{l}\text { Absence de fissuration } \\ \text { horizontale amont-aval } \\ \text { dans le noyau d'un barrage } \\ \text { zoné }\end{array} \\ \text { Filtration } & \begin{array}{l}\text { Absence d'érosion interne } \\ \text { dans le remblai }\end{array}\end{array}$

\section{Filtration}

F2

\section{Filtration}

F3

Drainage D1

Protection

P1

\section{Protection}

$\mathrm{P} 2$

\section{Protection}

P3

Évacuation

Ev1

Vidange

Ev3

Suivi de la sécurité

S1
Absence de fissuration suite au glissement, aux tassements et pérennité de l'étanchéité sous chargement sismique dans le remblai

Absence d'érosion interne dans la fondation

Absence d'érosion régressive au niveau du contact remblai fondation

Évacuation des fuites sans mise en charge des drains

Absence d'érosion en cas de déferlement de vagues

Absence d'affouillement en pied

Absence d'érosion

du parement amont

Évacuation de la crue de projet

Vidange de la retenue en cas de grand danger

Détection des effets

du vieillissement

sur l'étanchéité

et autres fonctionnalités projet

Par suite du séisme de base d'exploitation (SBE), l'étanchéité est dégradée et les fuites sont supérieures au débit de dimensionnement et inadmissibles pour la sécurité ou pour l'économie du projet

Apparition de contraintes effectives verticales de traction dans le noyau (fracturation hydraulique)

Non-vérification de la condition géométrique de rétention assurée par le filtre : règles de Terzaghi et de Delgado $\mathrm{K}_{\text {fittre }}<10^{-3} \mathrm{~m} / \mathrm{s}$

Conditions hydrauliques d'absence de détachement et de transport

Instabilité locale de la fondation en pied aval du barrage (déchaussement)

Débit insuffisant ou colmatage

Érosion causée par les vagues. Débit de déferlement causant l'érosion de la crête

Érosion causée par les vitesses d'écoulement

Érosion causée par les vagues lors de tempêtes

Surverse lors de la crue de

Perte d'évacuation ou évacuation insuffisante

Perte des moyens de détection de l'initiation des modes de rupture par érosion ou glissement
Estimation des déplacements irréversibles pouvant être engendrés par le SBE et vérification de leur admissibilité pour l'étanchéité et les filtres

Calcul en déformation pour vérifier que les contraintes effectives soient en compression dans le noyau

Dimensionnement de la granulométrie du filtre pour retenir les particules de l'étanchéité et limiter le débit notamment en crête du noyau suite à une fissure de dessiccation ou crue extrême

Limitation des gradients et des vitesses à la sortie de la fondation pour empêcher l'arrachement des particules ou filtre aval

Vérification de la stabilité locale en pied de digue

Vérification de la débitance du drain

Dimensionnement de la revanche pour que le débit de déferlement soit inférieur à un certain seuil suivant la nature du matériau et la nature du trafic sur la crête (y compris sécurité des accès)

Vérification que le matériau de protection ne soit pas emporté lors des crues

Vérification que les dégâts sur le matériau de protection soient acceptables lors des tempêtes

Dimensionnement de l'évacuateur et de la revanche pour évacuer la crue de projet avec laminage

Dimensionnement de la vidange de fond en fonction du risque aval (par exemple, vidange complète en $21 \mathbf{j}$. et demi-poussée atteinte en $8 \mathrm{j}$.)

Dimensionnement du plan de surveillance visuelle et d'auscultation en fonction du risque 
17.

\section{Modèle géologique de la fondation}

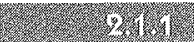 \\ Principes}

Les recommandations ci-après concernent les principaux points qu'il faut examiner lors des études géologiques des barrages et digues en remblai. Les objectifs du modèle géologique sont de fournir les informations nécessaires pour juger de la qualité de fondation : sa capacité portante, son étanchéité, la stabilité des appuis et les risques de tassements différentiels et d'érosion.

\section{- Géologie historique}

Toute analyse géologique doit d'abord prendre en compte dans quelles conditions et à quelle époque se sont mises en place les différentes formations qui vont servir de support à l'ouvrage projeté. Selon l'âge (récent ou très ancien) et l'environnement (milieu marin, alluvial, volcanique, magmatique, etc.), l'évolution des matériaux est différente (degré d'altération, fissuration, déformation). Ce travail est le plus souvent bibliographique.

\section{- Description des formations}

Le point le plus important est la parfaite connaissance de la nature lithologique du terrain en distinguant bien les terrains de couverture (avec présence éventuelle de niveaux compressibles) du substratum rocheux sous-jacent altéré et sain (qui peut d'ailleurs ne pas être sollicité lorsqu'il se trouve à une profondeur trop importante, dans le cas des digues à grand linéaire). Minéralogie et pétrographie sont la base du diagnostic en utilisant des termes normalisés et en évitant toute appellation locale pouvant conduire à des confusions.

\section{- Détermination des hétérogénéités}

Toutes les variations dans la nature et la structure des matériaux de fondation (altération, enclaves, bancs de nature et comportement différents, fracturation à toutes échelles, plissements, décollements, vides, remplissages postérieurs à la mise en place, etc.) sont précisées et expliquées. La géomorphologie est prise en compte pour analyser certaines formes du relief. L'ensemble de ces informations doit permettre de fixer les zones homogènes, les hétérogénéités et déboucher sur un modèle géologique du site concerné.

Il faut noter que toutes les hétérogénéités sont à étudier avec soin, car elles peuvent induire des comportements mécaniques particuliers (fissures lubrifiées par de l'argile, fissures en compression et fissures en distension par rapport à la contrainte tectonique régionale pour les barrages, lentilles argileuses compressibles ou chenaux perméables et drainants dans les terrains de couverture pour les digues en particulier)

\section{3.}

\section{Méthode pour obtenir un modèle géologique}

Un modèle géologique est, au minimum, une image du terrain en deux ou trois dimensions, montrant l'or- ganisation des différents objets géologiques les uns par rapport aux autres.

\section{1.}

\section{Enquête documentaire}

La recherche de toutes les données concernant un site est le préalable indispensable, en allant du général (études régionales) au particulier (rapports d'études spécifiques dans la zone du projet). Rappelons que certains sites ont pu faire l'objet d'études antérieures plus ou moins complètes, parfois oubliées. Pour les ouvrages anciens, on recherche en priorité les documents de projet et les plans de récolement.

\section{I.}

\section{Travail de terrain}

En préambule, nous dirons que le rôle du géologue est de lire et comprendre le paysage. Souvent il existe un support régional (en France la carte géologique au $1 / 50000$ ) qui sert de base de travail.

Quand on ne dispose pas de documents géologiques régionaux, une étude par stéréo-photo-interprétation permet de poser les choses, d'avoir une vision globale, même quand il y a peu d'affleurements, la géomorphologie étant alors d'un grand secours. Dans le cas des digues très longues, la photo-interprétation permet de retrouver, dans les grandes plaines alluviales, les anciens lits et méandres des rivières (paléo-chenaux) qui peuvent constituer autant de zones de circulations privilégiées (érosion interne) sous l'emprise de ces aménagements et qu'il faudra donc reconnaitre plus précisément pour les localiser.

Toute l'étude débouche sur un examen géologique du site avec levé d'une carte à une échelle appropriée (du 1/5000 ou 1/500 selon la précision souhaitée). On insiste particulièrement sur les terrains de couverture souvent absents des cartes générales.

Ensuite, sur les levés de détail, on fait figurer le maximum d'éléments géologiques permettant d'adapter l'ouvrage au site et de choisir les meilleures dispositions constructives : zones d'altérations, zones ou niveaux compressibles, fracturation avec une géométrie précise de tous les types de discontinuités du massif à toutes les échelles - microstructures à mégastructures - pouvant conduire à des phénomènes d'instabilité des versants et des fondations ou être des vecteurs de fuites pour les eaux de la retenue. On précise si les fractures sont ouvertes ou fermées ainsi que la nature de leur remplissage (étanche ou perméable) Sont également indiquées toutes les déformations plus souples (plis, microplis, étirements) qui sont les prémices des phases cassantes.

Si, pour un ouvrage en remblai, l'analyse structurale du massif est beaucoup moins poussée que pour un barrage en béton, on s'attarde néanmoins à vérifier l'orientation des fractures par rapport à l'axe de l'ouvrage et à la découpe des fouilles dans les versants. C'est lors de cette analyse que l'on voit les plans qui risquent de devenir instables et qui nécessiteront un 
soutènement (les terrassements peuvent débiter des plans particulièrement fragiles et entraîner, par exemple, des glissements ( banc sur banc »).

C'est aussi à cette phase que sont reportées toutes les informations concernant l'hydrogéologie (sources, suintements, niveaux perméables, niveaux étanches, faille drainante qui ferait communiquer l'amont et l'aval de l'ouvrage par exemple) ainsi que tous les éléments pouvant donner des idées sur une instabilité possible des versants (arbres, arrachement, forme du relief, zones humides, etc.). En conclusion, le levé de terrain doit être un document complet, précis et compréhensible pour les concepteurs, c'est un état des lieux le plus exhaustif possible.

Nous attirons l'attention sur le fait que ces travaux préparatoires à la mise au point d'un modèle géologique sont de plus en plus négligés et que l'on a tendance aujourd'hui à faire des extrapolations sommaires des documents géologiques existants. La carte au 1/50 000 régulière de la France, trop souvent agrandie, ne peut en aucun cas être un document de projet même pour les fondations d'un ouvrage en terre, moins exigeant que le béton, car les terrains de couverture sont rarement bien représentés.

C'est également lors de ces levés géologiques de détail qu'apparaissent les zones les plus favorables (topographiquement et géologiquement) pour l'implantation et le choix des ouvrages annexes. En effet, en particulier dans le cas des terrains sédimentaires et en fonction de l'azimut et du pendage des plans de la roche, un évacuateur de crues ou une galerie peuvent poser des problèmes de stabilité des fouilles selon l'orientation des couches par rapport au versant.

\section{Ther}

\section{Travaux de reconnaissance}

En aucun cas, il ne peut y avoir de standards, les conditions géologiques des sites étant beaucoup trop changeantes et différentes selon la nature des choses.

Les travaux de reconnaissance sont définis pour répondre à des questions précises après l'étude de surface et lever les inconnues qui subsistent. Les types de travaux doivent être choisis en fonction du contexte géologique et les méthodes doivent être adaptées à ce contexte.

\section{7.}

\section{Tranchées et puits}

Dans le cas des ouvrages en terre, les tranchées (ou puits) de reconnaissance réalisées à la pelle hydraulique sont d'emblée nécessaires quand il y a peu d'affleurements. Elles permettent souvent de positionner le contact couverture/substratum et de se faire rapidement une bonne idée du décapage, si la couverture meuble n'est pas trop importante (5 à $6 \mathrm{~m}$ au plus). Les tranchées sont la base des études de digues à grand linéaire, où les affleurements sont rares, masqués par les dépôts alluvionnaires.

\section{3.}

\section{Reconnaissances géophysiques}

Quand la couverture est trop épaisse, les tranchées sont avantageusement couplées à une campagne géophysique qui fait un lien entre elles (sismique surtout quand les contrastes de vitesse entre les formations sont bien marqués, électrique lorsqu'on veut différencier des formations meubles entre elles).

La sismique renseigne également sur la qualité globale du ( rocher », qu'il est très difficile d'apprécier de visu, en montrant, par la gamme de vitesses observées, l'importance de l'altération/décompression du massif par rapport à la roche saine. La sismique est le révélateur du degré de fracturation d'un massif rocheux. Elle est très utilisée pour les barrages.

Dans le cas de digues à grand linéaire, où les fondations sur terrains alluviaux sont le plus souvent meubles, avec présence d'une nappe, les méthodes électriques semblent, a priori, bien adaptées. On utilise d'abord des méthodes à grand rendement à savoir l'électromagnétique basse fréquence (champ proche, qui donne les zones plus ou moins conductrices, ou champ lointain, qui montre les hétérogénéités résistantes) et les panneaux électriques. La polarisation spontanée à grand rendement peut identifier des zones de fuite sur des remblais toujours en eau. Le radar géologique est aussi utilisé pour détecter des singularités, mais il offre une faible profondeur d'investigation dans les sols conducteurs. Toutes ces méthodes indirectes sont calées et contrôlées par des reconnaissances géotechniques (sondages, pénétromètres, etc.).

Les zones d'anomalies sont ensuite analysées par des méthodes géophysiques locales, puis par des méthodes géotechniques classiques.

\section{2.}

\section{Sondages}

Les sondages (avec une préférence pour les carottés, complétés par des destructifs avec enregistrement des paramètres de foration) renseignent sur la qualité du matériau en profondeur, permettent le calage de la géophysique et révèlent la structure fine du massif.

Les sondages servent bien évidemment pour des essais hydrauliques (Lefranc en terrains meubles et Lugeon en milieu rocheux) qui contribuent à définir un organe d'étanchéité adapté au projet à condition d'en faire un nombre suffisant, surtout pour les essais Lefranc, afin d'avoir une valeur un peu statistique des résultats. L'essai de pompage dans une formation perméable reste l'essai le plus précis pour quantifier la perméabilité à grande échelle.

Rappelons que pour les ouvrages en terre, les désordres et les désastres observés sont le plus souvent dus à des phénomènes d'érosion interne (du remblai, au niveau du contact fondation/sol, ou dans la fondation elle-même).

On ne peut pas se contenter de quelques essais systématiques si l'on veut vraiment cerner la perméabi- 
lité et concevoir le futur traitement d'étanchéité d'un milieu hétérogène (molasse par exemple où la cimentation des grains est très variable d'un point à l'autre et où l'on passe du sable au grès cimenté) et il faudra peut-être associer différents types d'essais pour une même formation.

Pour ce qui concerne les essais sur les matériaux, nous renvoyons à l'étude géotechnique stricto sensu.

Enfin, les reconnaissances sous les vieux ouvrages nécessitent parfois l'usage de sondages inclinés pour rechercher les conditions de fondations à l'aplomb de ces derniers. L'accès difficile, du fait de la disparition des pistes de chantier et de l'impossibilité de les rétablir simplement, demande alors l'usage de moyens importants.

\section{7.}

\section{Modèles géologique et hydrogéologique de la fondation}

A partir de ce qui a été vu précédemment, le géologue a en mains les éléments pour préparer les modèles géologique et hydrogéologique du site. La base de ces ( modèles ) est constituée des coupes géologiques interprétatives montrant en élévation la nature et la structure des formations présentes au droit de l'ouvrage ainsi que leur perméabilité.

On trouve essentiellement deux grands types de coupes :

- coupes lithologiques et structurales (modèle géologique) ;

- coupes des perméabilités et du comportement hydraulique des terrains (modèle hydrogéologique).

Dans le cas d'ouvrages existants, ceux-ci doivent figurer sur les coupes afin de bien visualiser le niveau de fondation et les traitements de sol réalisés.

\section{3.}

\section{Coupes lithologiques et structurales}

Elles doivent être faites sans distorsion des échelles verticales et horizontales, pour être correctement utilisables. Elles peuvent être transversales et longitudinales par rapport à l'axe de la vallée.

Elles doivent montrer :

- l'épaisseur des formations superficielles (alluvions, éboulis, colluvions) ;

- le substratum rocheux, s'il est atteint (en talweg en particulier) ;

- l'épaisseur du rocher altéré (bien visible sur les carottes), qui peut parfois être considéré comme un sol (arènes granitiques par exemple) ;

- l'épaisseur du rocher décomprimé. Rappelons à ce propos que les traces d'altération et d'oxydation restent le meilleur témoin de la décompression mécanique des roches en profondeur, information négligée le plus souvent. Dans les cas les plus favorables, elles se calent parfaitement sur une gamme de vitesse sismique, permettant ainsi de très bonnes corrélations mécaniques pour des tranches de terrain bien marquées. Il est en effet très rare de pouvoir matérialiser ainsi un horizon sismique ;

- les variations de nature des roches et des sols dans le cas de terrains sédimentaires, avec une attention particulière à la différenciation entre les niveaux compressibles et résistants (digues) ;

- la position exacte du substratum sain et compact, bien que, dans le cas des barrages en remblai, celui-ci soit rarement recherché pour y asseoir l'ouvrage ;

- l'importance de la fracturation (analyse structurale, analyse de l'indice RQD $\$ 2.4 .3 .4$ );

- la structure du site basé sur des corrélations de sondage à sondage et des éléments de déformations simples à très complexes (plis, étirements, chevauchement, variation dans la sédimentation avec des niveaux ou lentilles de qualité médiocre).

Elles doivent permettre de définir :

- le choix du meilleur axe et le type d'ouvrage le plus approprié, compte tenu de la qualité mécanique du support;

- l'importance du décapage, du parafouille ou de la clé d'ancrage nécessaire, des sur-profondeurs locales adaptées à la réalité du terrain (pour les digues en particulier) :

- les zones particulières où des confortements pourraient être nécessaires à l'ouverture des fouilles (problème de stabilité dans des roches sédimentaires stratifiées) ;

- le type, l'orientation et la profondeur de ces confortements ;

- les zones où des purges seraient indispensables pour conserver une fondation acceptable (tassements différentiels) ;

- le choix de l'implantation des ouvrages associés qui sont intimement liés à la géométrie, mais surtout aux conditions géologiques qui peuvent s'avérer très pénalisantes dans les cas défavorables.

\section{I.}

\section{Coupes des perméabilités et du comportement hydraulique du terrain}

Elles sont réalisées de préférence au droit du futur organe d'étanchéité de l'ouvrage (sur l'axe dans le cas d'une paroi, en pied amont dans le cas d'un masque, sur tout le développement d'une digue à grand linéaire) et sans distorsion d'échelles (sinon elles ne permettent pas une vision réaliste du site).

Rappelons que les perméabilités en milieu rocheux sont exprimées en $\mathrm{l} / \mathrm{min} / \mathrm{m}$ à la pression que l'on a choisi de représenter (si possible celle du futur plan d'eau) et que les perméabilités dans les sols meubles sont représentées en $\mathrm{m} / \mathrm{s}$.

Avec cela, on dresse une (ou des) coupe(s) synthétique(s) des perméabilités reliant tous les sondages (en précisant le niveau de la nappe, les sondages ayant été aussi équipés en piézomètres). 
Cette coupe doit montrer :

- l'épaisseur de la tranche perméable ;

- la profondeur/TN du terrain étanche ou considéré comme tel ;

- les zones avec anomalies de perméabilité ;

- la limite d'oxydation de la roche témoignant des circulations d'eau, donc de la décompression dans le cas des roches (ouverture partielle des fissures).

Cette synthèse est complétée par des éléments d'analyse tels que :

- le comportement des essais entre la montée et la descente en pression pour un même palier de pression (débourrage/colmatage/comportement équivalent), à partir de l'analyse des courbes d'absorption d'eau ;

- les pertes totales d'eau de foration, en expliquant leur raison ;

- les contournements d'obturateur et leurs causes probables.

Cela permet de définir, en fonction de la nature du terrain :

- la limite à partir de laquelle la perméabilité est acceptable en milieu fissuré (critère de Lugeon pour les barrages $>50 \mathrm{~m}$ ), absorption inférieure ou égale à $2 \mathrm{l} / \mathrm{min} / \mathrm{m}$ sous $0,5 \mathrm{MPa}$ (pour les barrages $<50 \mathrm{~m}$ ), la limite de perméabilité acceptable en milieu pulvérulent (fondations sur des alluvions) fournie par des essais de type Lefranc, de pompage, Nasberg, etc.

- la géométrie en fondation des zones très perméables par rapport aux zones peu perméables ou étanches (contraste des perméabilités);

- la géométrie du voile normal d'injection ou de l'organe d'étanchéité retenu (on s'encastre généralement de 3 à $5 \mathrm{~m}$ dans le terrain étanche lors des injections) et du voile au large pour les barrages;

- les zones où une étanchéité particulière (plus de lignes d'injection, forages plus rapprochés, clé en matériaux étanches, parois, etc.) est nécessaire ;

- l'orientation et l'inclinaison des forages par rapport à la structure d'ensemble de façon à recouper le maximum de plans de fractures défavorables par leur orientation à l'étanchéité générale (plans amont-aval ouverts, donc naturellement drainants) pour les barrages, l'orientation et la profondeur de coupures en matériaux étanches pour les digues;

- la géométrie et l'importance du voile de drainage qui doit tenir compte de celles décidées pour le voile d'injection. Rappelons que la maîtrise du drainage (donc des circulations d'eau) est un des points les plus importants de la sécurité des barrages en remblai et des digues ;

- se faire une idée du futur réseau d'auscultation (piézomètres implantés dans les zones critiques).

Pour les barrages anciens, tous les organes d'auscultation mis en place antérieurement doivent être calés par rapport aux données géologiques fournies, de façon à vérifier leur efficacité et leur rôle réel.

A noter que, dans le cas des barrages, tout ce qui vient d'être dit doit également être repris pour l'étude de la cuvette. Celle-ci peut parfois poser indirecte- ment de sérieux problèmes vis-à-vis de la stabilité de l'ouvrage (écroulement de versant, communication cuvette/site par des réseaux de fractures orientés amont-aval et développement de phénomènes d'érosion interne en fondation ou dans l'ouvrage luimême).

\section{4.}

\section{Conclusion}

L'étude géologique appliquée aux barrages et aux digues en remblai, qui ont un comportement plus ( souple ) que les autres ouvrages vis-à-vis de leur support naturel, reste un temps fort et incontournable des études, à l'amont de toute autre intervention.

Le géologue doit travailler à toutes les phases du projet afin de fournir au concepteur un modèle (ou une image) géologique du site de plus en plus précise et proche de la réalité au fur et à mesure que les études avancent. On limite ainsi les risques de mauvaises surprises à l'ouverture des fouilles, dont les conséquences pèsent, généralement, très lourd sur le coût final de l'aménagement.

Les modèles géologique et hydrogéologique du site (le plus souvent des coupes en 2D) sont une synthèse de données qualitatives et quantitatives dono hétérogènes, faite de corrélations visuelles (nature changeante des terrains), hydrauliques (perméabilité très variable d'un point à un autre), mécaniques (ensembles regroupés sous des caractéristiques les plus homogènes possibles, sachant qu'il faut faire des classements avec toutes les incertitudes qui peuvent malgré tout subsister).

Enfin, on attache une attention particulière aux points-clés vis-à-vis de la stabilité géologique de la fondation d'un ouvrage en remblai :

- l'écoulement, le cheminement et la maîtrise des eaux dans la fondation par rapport à la structure géologique d'ensemble. On peut, en effet, aboutir à d'importants désordres ultérieurs si cela n'a pas été parfaitement maîtrisé en temps voulu ;

- l'examen des conditions de stabilité initiale naturelle de la zone d'implantation de l'ouvrage, mais aussi l'influence de la modification de ces conditions lors de l'ouverture des fouilles du barrage. Ce point peut être très critique pendant le chantier;

- l'existence de terrains compressibles ou déformables sous l'emprise de l'ouvrage en envisageant soit leur décapage en s'assurant parfaitement de leur extension, soit leur conservation en prenant des dispositions constructives appropriées ;

- le choix et le positionnement des ouvrages associés pour les barrages, en tenant compte de la réalité géologique du site et en évitant de recouper des formations qui favoriseraient des instabilités ultérieures;

- pour des reprises sur de vieux ouvrages, les travaux et ouvrages réalisés sont recalés, dans la mesure du possible, par rapport au contexte géologique du site, surtout en l'absence de plans de récolement antérieurs. 


\section{Géomorphologie fluviale (cas des digues)}

Les évolutions et processus géomorphologiques des cours d'eau peuvent avoir un impact sur la sécurité des digues par divers processus (Degoutte, 2006) :

- érosion des berges par le courant pouvant déstabiliser des digues proches du lit mineur;

- évolution du tracé en plan pouvant modifier les courants en crue ou rapprocher le lit mineur de la digue ;

- évolution du profil en long par enfoncement localisé ou généralisé (incision) pouvant déstabiliser des digues proches par glissement, diminuer la fréquence des surverses, changer la localisation des premières surverses ou le fonctionnement des déversoirs ;

- évolution du profil en long par exhaussement localisé ou généralisé pouvant également modifier les conditions de surverse.

Ces processus sont conditionnés par le transport solide des matériaux alluvionnaires, mais on n'oubliera pas également l'importance de la végétation ligneuse aussi bien pour son rôle sur la résistance des berges que pour le transport de bois flottant et les formations d'embâcles. Le bois transporté peut être un agresseur des berges, des talus de digues ou des protections de ces talus. Le bois déposé en crue peut occasionner des zones de surverses localisées.

Les processus d'évolution généralisée du profil en long sont l'érosion progressive, l'érosion régressive, l'exhaussement progressif et l'exhaussement régressif. L'incision désigne le résultat d'une érosion régressive ou progressive. L'incision est généralement due à une cause extérieure à l'endiguement, mais peut être également provoquée par la présence des digues resserrant un lit mineur. Les processus localisés sont des érosions ou des dépôts, en particulier dans les coudes. L'affouillement des berges désigne une érosion du pied.

Dans le cas des rivières à méandres de pente forte, les processus d'évolution du tracé sont des reptations et des coupures naturelles de méandres par déversement. Pour les rivières à méandres de pente très faible, les processus sont des migrations latérales et des coupures par tangence. Dans le cas des rivières en tresses et des rivières à style divaguant (wandering), les évolutions sont des déplacements, des créations ou des comblements de bras.

Les digues sont d'autant plus susceptibles d'être impactées par tous ces processus qu'elles sont proches du cours d'eau. La présence ou l'absence d'un espace entre lit mineur et digue (franc-bord ou ségonal) conditionne la nécessité d'un examen morphologique plus ou moins approfondi.

Les objectifs de l'étude géomorphologique sont de fournir les informations nécessaires pour juger de la sécurité de l'implantation et des sollicitations dans les situations de crue. Elle s'attache en particulier à définir un fuseau de mobilité du cours d'eau et une appréciation des vitesses d'évolution dans ce fuseau, qui peuvent être très rapides, à l'échelle de la crue ou au contraire extrêmement lentes, à l'échelle humaine.
Les documents cartographiques ou photographiques (cartes IGN récentes, cartes d'État-major, cartes de Cassini, plans cadastraux récents, cadastre napoléonien, cartes géologiques, photographies aériennes) permettent une approche historique de la mobilité en plan d'une rivière. Ils permettent tout à la fois d'apprécier la vitesse des évolutions de tracé et de connaitre leur sens. L'étude morphologique doit aussi comporter une approche de terrain (observation de la présence de pavage, recherche d'anciens chenaux...).

L'étude géomorphologique doit comporter une étude de la granulométrie des alluvions afin de compléter la connaissance de la dynamique du cours d'eau. La connaissance géologique $(\$ 2.1)$ indique la profondeur du substratum rocheux, ce qui conditionne la possibilité que des mécanismes d'incision se développent ou non. Une rivière atteignant le substratum est d'avantage susceptible de connaître des évolutions latérales.

Enfin, une étude géomorphologique doit aussi comporter une analyse de la végétation dans la zone concernée par l'aménagement, compte tenu de son impact sur la résistance des berges à l'érosion par le courant ou au glissement. Cette étude doit aussi s'intéresser au boisement du lit majeur en amont qui conditionne le potentiel de transport d'arbres arrachés et donc la création d'embâcles. La présence sur le secteur de l'aménagement de pièges potentiels pour les embâcles (ponts, seuils, coudes prononcés) et la recherche de témoignages permettent aussi de préciser les secteurs concernés.

\section{3 \\ Modèle géométrique}

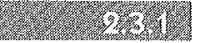 \\ Objectif}

L'objectif du modèle géométrique est d'obtenir une représentation simplifiée de la géométrie de l'ouvrage conservant l'information pertinente pour le problème étudié. Le plus fréquemment, cette représentation consistera en la sélection judicieuse de sections transversales de l'ouvrage.

\section{2. \\ Choix du profil}

Le choix de ces sections sera fait à l'issue de l'analyse des données topographiques et de l'analyse géologique. Il existe autant de sections à calculer que de coupes-types de remblai et de faciès géologiques de fondation.

\section{9.}

\section{Cas des ouvrages anciens}

Pour cette catégorie d'ouvrage, le choix de la section sera décidé à partir de l'étude des plans de récolement, des mesures d'auscultation et des observations de la reconnaissance visuelle. Il existe autant de sections à calculer que de types de comportement observé. 
L'expérience acquise sur les ouvrages les plus anciens montre qu'il existe souvent des écarts entre plans et réalité :

- erreurs ;

- changement de référentiel ;

- manque de récolement ;

- tassement des ouvrages.

Si des doutes subsistent quant à la géométrie actuelle de l'ouvrage, il ne faut pas hésiter à recourir à des levés topographiques complémentaires pour les besoins spécifiques du modèle hydraulique.

\section{4}

\section{Modèle géotechnique de la fondation et du remblai}

\section{8}

\section{Principes}

Le modèle géotechnique a pour objectif de définir un cadre de représentation des propriétés de résistance et de déformabilité de l'ouvrage et de ses fondations, de manière à évaluer le plus correctement possible son comportement et sa sécurité vis-à-vis des différentes états limites. site :

La constitution d'un modèle géotechnique néces-

- d'identifier les mécanismes auxcuels on va s'intéresser et leurs états limites associés, et d'y associer des lois de comportement adaptées et des modèles d'états limites ;

- d'identifier les matériaux constitutifs du remblai et des fondations ;

- d'évaluer au mieux, au moyen de valeurs dites « caractéristiques » qu'il conviendra de déterminer avec soin, les propriétés géotechniques des matériaux constitutifs du remblai ou des fondations intervenant dans les lois rhéologiques et les conditions d'états limites.

Le modèle géotechnique est établi en lien avec le modèle géométrique et le modèle géologique. Il conditionne le modèle hydraulique.

L'objet de ce chapitre est de guider le concepteur dans l'identification et l'évaluation des propriétés géotechniques du remblai et de la fondation, notamment leurs valeurs caractéristiques. On distingue pour cela, d'une part les matériaux meubles qui constituent les sols en place de la fondation, d'autre part les sols mis en œuvre dans le remblai proprement dit, enfin les matériaux rocheux de fondation.

\section{6.).}

\section{Les propriétés géotechniques du remblai et de sa fondation}

Les états limites identifiés conduisent à orienter le choix des propriétés géotechniques des sols à déterminer. Ces dernières sont appréhendées par la démarche suivante :
- l'identification des matériaux;

- la mesure du comportement mécanique du sol ;

- le choix des propriétés en fonction des états limites à évaluer.

\section{3.}

\section{Identification}

\section{4.}

\section{Identification des sols et des roches : principes}

L'identification des sols et des roches est basée sur des essais de laboratoire et des critères visuels objectifs, faisant l'objet de protocoles normalisés, de manière à permettre leur classement dans une catégorie reconnue par l'ensemble de la profession. La description doit être complète, l'appellation précise et les propriétés non ambiguës.

Les paramètres d'identification constituent des données importantes, car ils permettent de :

- relier le matériau étudié à une classe de sols dont les propriétés varient dans une plage connue ;

- utiliser des corrélations à caractère général les reliant à des paramètres mécaniques.

Pour les ouvrages hydrauliques en remblai, on peut utiliser plusieurs classifications : la classification GTR pour les travaux de terrassement routiers, la classification LCPC, la classification USCS, etc. La classification GTR permet d'appréhender les problématiques du réemploi des matériaux en remblai et des terrassements.

\section{2.}

\section{Identification des sols meubles (fondation, remblai)}

Les principaux paramètres d'identification sont: - la teneur en eau naturelle $w_{\text {nat }}$ et le degré de saturation $\mathrm{S}_{\mathrm{r}}$ (norme NF P94-050) ;

- la granulométrie et la sédimentométrie (norme NF P 94-056 et 057) ;

- les limites d'Atterberg (norme NF P 94-051) ou Valeur

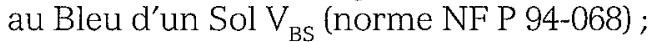

- la blocométrie (norme NF EN 13393).

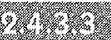

\section{Identification des agrégats et enrochements}

Les essais concernent essentiellement les matériaux d'emprunts, y compris les agrégats et les enrochements. Ils sont notamment un élément de reconnaissance des roches évolutives sous l'action de l'eau et des contraintes.

Le guide de référence relatif aux enrochements et leurs utilisations dans les ouvrages hydrauliques est The Rock Manual ${ }^{1}$. Les principaux paramètres qui peuvent être déterminés sur les matériaux rocheux sont les suivants :

(1) En versions anglaise et française (voir bibliographie). 
- Paramètres intrinsèques

- densité apparente ;

- minéralogie.

- Paramètres d'état

- teneur en eau ;

- granulométrie, blocométrie ;

- coefficient de forme ;

- porosité.

- Paramètres de résistance

- essai Los Angeles (LA) et essai d'usure Micro-Deval (MDE) (norme NF EN 1097-2 et 1097-1) : ils estiment respectivement la résistance d'un matériau rocheux à la fragmentation par chocs et la résistance à l'usure. Les coefficients LA et MDE sont utilisés pour les roches relativement dures : granite, gneiss, calcaire et grès dur;

- essai de fragmentabilité des matériaux rocheux (FR) (norme NF P 94-066) : il détermine l'aptitude d'un matériau rocheux à se fragmenter sous l'effet des sollicitations mécaniques qu'il subit lors de son compactage. L'interprétation du coefficient FR vise à caractériser les matériaux rocheux évolutifs et plus ou moins friables, pour lesquels les coefficients LA et MDE ne permettent pas toujours d'extrapoler la résistance des petits agrégats ;

- essai de gélivité (norme NF EN 12371) : il détermine la résistance à l'usure par des cycles de gel et dégel pour les pierres naturelles. Il est utilisé par exemple pour vérifier la résistance au gel des enrochements mis en place en protection de parement amont des barrages d'altitude ;

- essai de dégradabilité des matériaux rocheux (DG) (norme NF P 94-067) : il détermine l'aptitude d'un matériau rocheux à se dégrader sous l'action combinée des agents climatiques ou hydrogéologiques (gel, cycles imbibition-séchage) et des contraintes mécaniques. Il est utilisé pour caractériser la dégradabilité des roches argileuses, telles que les marnes, les argilites et les schistes sédimentaires.

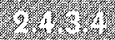

\section{Identification du rocher de fondation}

Le rocher est identifié par :

- ses propriétés intrinsèques (densité apparente et minéralogie) ;

- la mesure de fissuration/fracturation : l'indice RQD (Rock Quality Designation) est une estimation quantitative de la fracturation influençant le comportement de la masse rocheuse ;

- la combinaison de paramètres descriptifs de la résistance du massif fracturé : caractérisation de la fracturation, de l'état des joints (rugosité, altération, nature du remplissage...). A titre d'exemples, peuvent être citées les méthodes suivantes :

- Tunneling Quality Index, Barton, 1974,

- Rock Mass Rating, Bienawski, 1976,

- Geological Strength Index, Hoek et Brown, 1995.

\section{3.}

\section{Les propriétés géotechniques utilisées pour le calcul}

Les propriétés géotechniques que l'on va chercher à caractériser sont de différentes natures :

- poids volumique (\$ 2.4.4.1) ;

- propriétés mécaniques : déformabilité (\$2.4.4.2 et 2.4.4.3) et résistance au cisaillement (\$ 2.4.5);

- propriétés hydrauliques : conductivité hydraulique (§ 2.4.4.4) et potentiel de développement de pression interstitielle ( $§ 2.4 .8 .5$ ).

Les aspects mécaniques et hydrauliques doivent être abordés conjointement dans le cadre de la justification du dimensionnement d'un projet.

\section{4.}

\section{Poids volumique}

On distingue divers types de poids volumiques : poids volumique sec, poids volumique humide, poids volumique saturé, poids volumique immergé (ou déjaugé). Il convient d'être attentif à l'emploi du poids volumique bien adapté à la représentation du matériau.

L'usage est de caractériser :

- les matériaux en place (fondation, remblai existant) par le poids volumique humide du sol en place ;

- les matériaux d'emprunt (remblai à construire) par la teneur en eau et le poids volumique sec à l'Optimum Proctor Normal ( $\mathrm{w}_{\mathrm{OPN}}$ et $\boldsymbol{\gamma}_{\mathrm{dOPN}}$ ).

\section{3.}

\section{Déformabilité, élasticité : modules}

Ces paramètres sont issus de la théorie de l'élasticité, applicable aux sols. Les précautions, liées aux divergences qui existent entre la théorie de l'élasticité et le comportement réel des sols qui sortent de leur domaine (célastique » dès que les déformations dépassent un certain seuil, doivent être intégrées :

- la courbe contrainte/déformation d'un sol n'étant pas linéaire, la valeur du module d'élasticité varie avec la déformation, selon la gamme de contraintes dans laquelle on se place : il faut donc être attentif à associer à une valeur de module d'élasticité son domaine de validité en termes de déformations et de contraintes;

- la déformation résultant de l'application d'une contrainte n'est pas réversible ;

- la vitesse de chargement influence la réponse du sol : des efforts dynamiques (vibrations) produiront des effets différents d’un chargement de longue durée.

Les deux paramètres caractérisant le comportement élastique sont le module d'élasticité E (également appelé module d'Young) qui traduit le rapport entre le déviateur de contraintes $\left(\sigma_{1}-\sigma_{3}\right)$ et la déformation principale $\varepsilon_{1}$ du sol, et le coefficient de Poisson $v$. Selon les conditions, il conviendra de différencier :

- le module drainé E, associé au coefficient de Poisson drainé $v$, lorsque le phénomène de consolidation a le temps de se produire. Selon le modèle rhéologique utilisé, on utilise une unique valeur de E pour les modèles avec élasticité linéaire ou plusieurs valeurs pour 
les modèles à élasticité non linéaire $\left(\mathrm{E}_{\mathrm{i}}\right.$ module initial, $\mathrm{E}_{\mathrm{t}}$ module tangent, $\mathrm{E}_{\mathrm{s}}$ module sécant, $\mathrm{E}_{\mathrm{ur}}$ module de déchargement-rechargement...);

- le module non drainé $E_{u^{\prime}}$ associé au coefficient de Poisson non drainé $v_{u}$, lorsque les contraintes sont appliquées rapidement et que le phénomène de consolidation, compte tenu de la perméabilité des sols et des conditions de drainage, n'a pas le temps de s'établir (approche en contraintes totales) ;

Signalons que ce sont généralement les paramètres drainés $(E, v)$ qu'il faut utiliser, même pour le calcul du comportement non drainé, les logiciels ajoutant automatiquement un module de compressibilité pour l'eau et distinguant les contraintes totales, les contraintes effectives et les surpressions interstitielles ;

- le module dynamique $E_{d}$ (les modules $E$ et $E_{u}$ précédents étant des modules statiques). Il correspond à la réponse du sol sous des sollicitations très brèves telles que celles produites par les machines vibrantes ou les séismes. Il s'agit d'un module non drainé et l'on considère généralement que sa valeur est au moins trois fois supérieure à $E_{u}$

On définit également un module de cisaillement $G$, qui traduit le rapport entre contraintes tangentielles $\tau$ et distorsion $\theta$ subie par le sol. Ce module a la même valeur, que l'on se trouve en condition drainée ou non drainée. Il est lié à E, via le coefficient de Poisson drainé, par la relation suivante :

$$
G=\frac{E}{2(1+v)}
$$

Le module d'élasticité drainé E et le module œdométrique (cf. § 2.4.4.3) sont également reliés, via le coefficient de Poisson drainé :

$$
E=E_{\text {oed }} \cdot \frac{(1+v) \cdot(1-2 v)}{(1-v)}
$$

Les paramètres de déformabilité sont essentiellement utilisés dans les calculs aux éléments finis ou dans des formules empiriques de calcul de tassement.

\section{$94+445$}

\section{Déformabilité et consolidation : œdomètre}

Lorsque l'on souhaite évaluer le tassement selon la méthode du chemin de contrainte, on s'appuie sur les résultats issus de l'essai œdométrique qui permet de déterminer, pour les sols fins :

- la contrainte de consolidation $\sigma_{\mathrm{c}}^{\prime}(\mathrm{en} \mathrm{kPa})$;

- l'indice de compression $\mathrm{c}_{\mathrm{c}}$;

- le module odométrique $\mathrm{E}_{\mathrm{ced}}(\mathrm{en} \mathrm{kPa})$;

- le coefficient de consolidation $\mathrm{C}_{\mathrm{v}}\left(\mathrm{en}^{2} / \mathrm{s}\right)$.

Ces paramètres servent à l'évaluation des tassements et de leur évolution dans le temps (tassements instantanés et différés).

\section{Paramètres hydrauliques}

Le principal paramètre hydraulique est la perméabilité (ou conductivité hydraulique) du remblai et de la fondation.
Ce paramètre est difficile à caractériser :

- il varie de plusieurs ordres de grandeur en fonction de la nature et de l'état des sols et matériaux rencontrés ; il est largement influencé par les défauts et irrégularités géotechniques ou de construction ;

- il varie dans le temps car il dépend étroitement de l'état de saturation du sol.

Dans un milieu saturé, il existe des formules qui donnent une estimation de la perméabilité en fonction

\begin{tabular}{|c|c|c|}
\hline $\begin{array}{l}\text { Perméabilité } \\
\text { dans le sol }\end{array}$ & $k=n \frac{R^{2}}{8} \frac{\rho g}{\mu}$ & $\begin{array}{c}n: \text { porosité } \\
\mu=\text { viscosité cinématique } \\
10^{-3} \text { Poiseuille à } 20^{\circ} \\
R \text { : rayon du capillaire } \\
\text { moyen do sol en } \mathrm{m}\end{array}$ \\
\hline $\begin{array}{c}\text { Perméabilité } \\
\text { dans. } \\
\text { la roche }\end{array}$ & $k=n \frac{e^{2} \rho g}{12 \mu}$ & $\begin{array}{c}\rho: \text { masse volumique } \\
\text { en } \mathrm{kg} / \mathrm{m}^{3} \\
g: \text { accélération } \\
\text { de la gravité } \\
\text { e : épaisseur de la fissure } \\
\text { moyenne en m }\end{array}$ \\
\hline
\end{tabular}
de la taille des vides.

Des formules pratiques (Hazen, Vaughan, Kozeny) sont données en annexe 2.

Pour les sols et remblais des ouvrages réels, il faut distinguer perméabilité horizontale et perméabilité verticale. La perméabilité horizontale est généralement 5 à 100 fois plus forte que la perméabilité verticale, en raison de la stratification des dépôts naturels et en raison de la construction par couches des remblais.

Lorsqu'un sol est composé de couches multiples, il est parfois possible de considérer une perméabilité homogène équivalente (cf. annexe 2).

Le comportement des ouvrages, en particulier des digues fluviales, est fortement conditionné par le comportement hydraulique des sols non saturés. Des modèles de comportement en conditions de sol non saturé sont mis en œuvre dans plusieurs logiciels commerciaux. L'annexe 2 précise les concepts et formules les plus souvent utilisés pour les calculs hydrauliques en milieu poreux non saturé.

\section{1.}

\section{Résistance au cisaillement}

\section{3.}

\section{Généralités}

Pour un matériau donné, sur une représentation dans le plan de Mohr $(O, \sigma, \tau)$, la courbe intrinsèque sépare le domaine des contraintes admissibles des contraintes provoquant la rupture. Dans le cas général des sols, cette courbe intrinsèque (ou critère de rupture) est non linéaire. Cependant, en première approximation, on considère que cette courbe peut être assimilée, au moins pour un intervalle donné de variation des contraintes, à une droite appelée droite de Coulomb. De cette approximation découle le critère de rupture de Mohr-Coulomb qui est de la forme : $\tau=\mathrm{c}+\sigma \tan \varphi$. 
D'autres formes de courbes intrinsèques peuvent être mieux adaptées pour décrire la résistance de certains sols $(\S 2.4 .5 .4$ pour les sols fins surconsolidés et $\S 2.4 .5 .5$ pour les enrochements).

\section{Whis}

\section{Un sol, plusieurs critères}

L'approche de la résistance au cisaillement des sols est appréhendée de manière différente selon la nature des matériaux et l'on distingue fondamentalement deux familles : les sols fins et les sols granulaires, la distinction étant liée à leur comportement différent sous sollicitation.

Les sols fins, caractérisés par de très faibles valeurs des coefficients de perméabilité, présentent un comportement différent à court terme (ou en conditions non drainées, avec des déformations à volume constant) et à long terme (ou en conditions drainées, subissant des déformations de consolidation ou de gonflement). Le passage du court terme au long terme s'effectue en un temps plus ou moins long appelé « temps de consolidation $)$, qui dépend des coefficients de consolidation des sols et de la géométrie des couches.

Les sols granulaires, caractérisés par une perméabilité élevée, ont en revanche en permanence un comportement de long terme, ou drainé, sauf dans le cas particulier des sols fins granulaires sous sollicitations dynamiques (séisme).

Un même sol peut être caractérisé par plusieurs critères de résistance. On distingue, pour une contrainte de confinement donnée (Fig. 2.1) :

- la résistance maximale (ou résistance de pic) : c'est le plus fort cisaillement qu'un sol peut supporter $\left(\tau_{\text {max }}\right)$; jusqu'à $\tau_{\text {max }^{\prime}}$ la structure initiale du sol n'est pas affectée ;

- la résistance critique $\left(\tau_{\text {lim }}\right)$ : cisaillement que le sol supporte lorsqu'il a atteint son état (c critique ), état stabilisé post-pic pour certains sols (sables denses, argiles sur-consolidées) ou état asymptotique pour les sols lâches ou remaniés ;

- la résistance résiduelle : le cisaillement que le sol supporte lorsqu'il a subi de grands déplacements sur des surfaces de ruptures, qui ont pu provoquer un lissage du plan de rupture, c'est-à-dire une réorientation des grains ou plaquettes d'argile selon ce plan.

La résistance maximale peut être beaucoup plus forte que la résistance critique et résiduelle pour les sols denses, surconsolidés et pour les sols indurés. Selon leur densité initiale, les sols ont un comportement dilatant ou contractant lors d'un essai de cisaillement, ainsi qu'illustré sur la figure 2.2.

Selon le type de sol et le problème géotechnique posé, on s'intéresse à l'une ou l'autre de ces résistances:

- la résistance maximale : il convient alors de vérifier que la déformation est bien restée en deçà de la déformation de pic (éventuellement calcul en déformation, ou a minima vérification de la compatibilité de la déformation des matériaux) ;

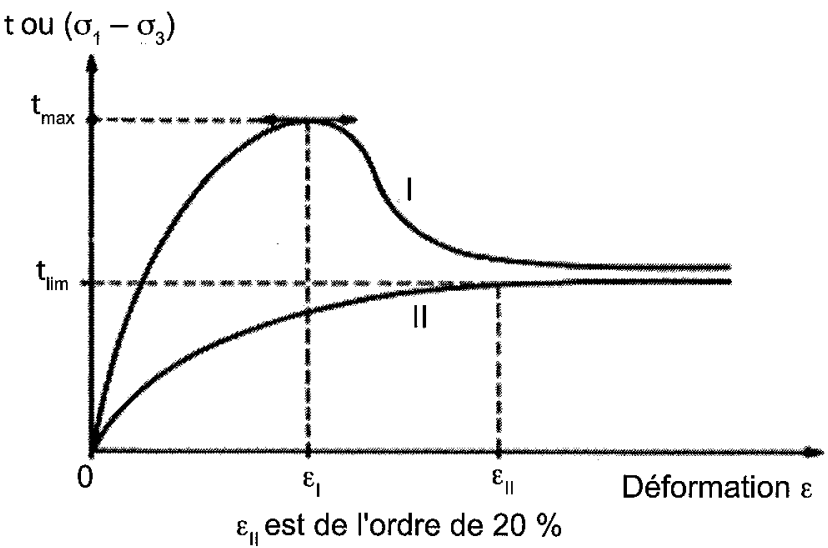

16. 21 Courbes contraintes-déformations pour des sols surconsolidés (I) : comportement dilatant ; et normalement consolidés (II) comportement contractant.

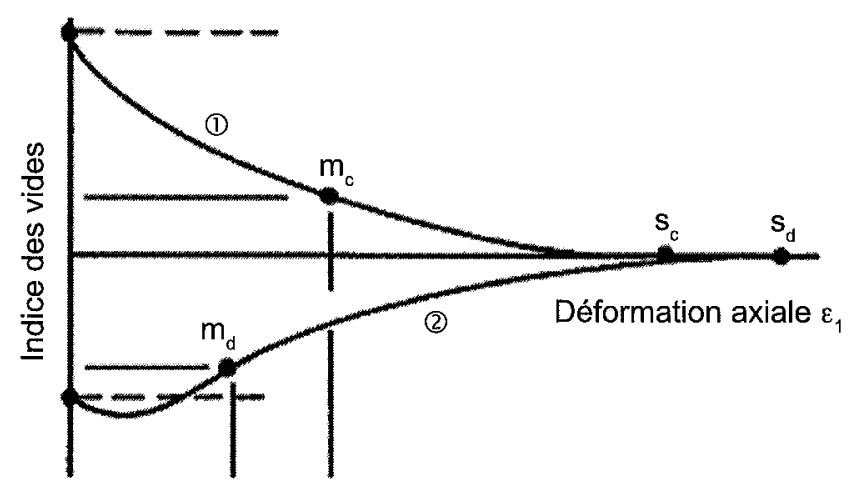

16. 22. Comportement dilatant et contractant des sols. Variation de l'indice des vides. 1) sol contractant ; 2) sol dilatant (Poulos, 1971).

- la résistance critique, voire résiduelle, lorsque des matériaux de déformabilité très différente interviennent dans le calcul, ou lorsque des déformations importantes sont attendues ou bien encore lorsque des plans de cisaillement ont déjà été mobilisés dans le remblai ou dans la fondation.

\section{4.}

\section{Résistance au cisaillement des sols à court terme}

Les matériaux fins saturés, en situation non drainée (ou court terme au regard du temps nécessaire à leur consolidation) présentent, pour une contrainte de consolidation donnée, une résistance au cisaillement liée uniquement à la cohésion $\mathrm{C}_{u^{\prime}}$ l'angle de frottement interne $\varphi_{u}$ étant nul.

Si les matériaux ne sont pas saturés, la résistance au cisaillement présente une valeur inférieure, qui dépend à la fois de la cohésion $c_{u u}$ et de l'angle de frottement interne $\varphi_{\mathrm{uu}}$ non nul.

Ceci se résume par la courbe intrinsèque d'allure suivante (Fig. 2.3) : 


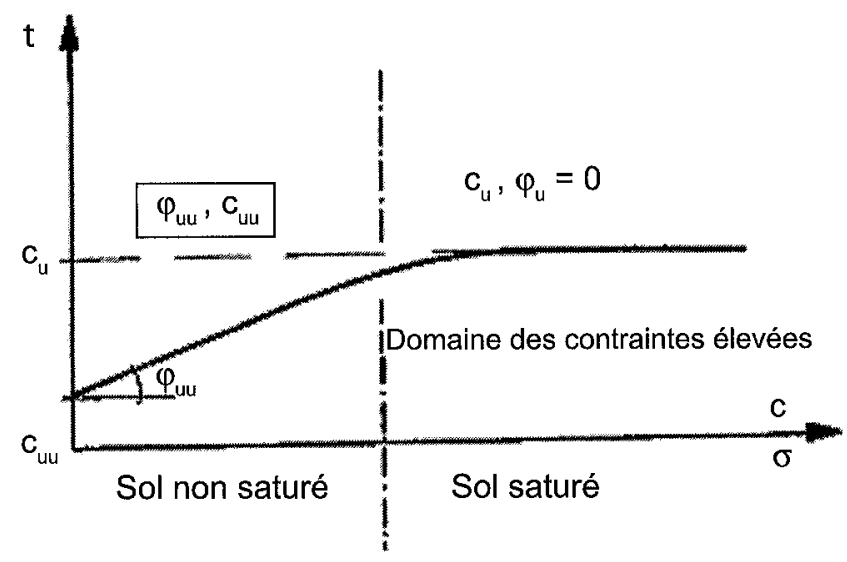

16.623 Critère de rupture en conditions non drainées (Philipponnat, 2006).
Dans la plupart des cas, il faut considérer l'angle de frottement apparent non drainé comme nul $\left(\varphi_{u}=0\right)$. Les exceptions sont les suivantes:

- les sols indurés, saturés ou non ;

- les sols non saturés, de module faible à moyen, dans un domaine restreint de contraintes normales. Cette particularité est rarement exploitée dans les applications.

\section{2.t.}

\section{Résistance au cisaillement des sols à long terme}

A long terme, l'évaluation de la cohésion est toujours délicate. On peut retenir quelques principes.

- Pour les sols grenus et les sols fins normalement consolidés ou faiblement surconsolidés, la cohésion c' est faible, voire nulle.

- Pour les sols fins surconsolidés, il faut distinguer deux domaines (Fig. 2.4) :

- le domaine normalement consolidé, pour les fortes contraintes normales, au-delà de la pression de consolidation $\sigma^{\prime}$. Il lui correspond une droite de rupture passant par $1^{\prime}$ origine $\left(\mathrm{C}^{\prime}=0\right)$;

- le domaine surconsolidé, en deçà de $\sigma_{p}^{\prime}$. L'enveloppe qui lui correspond peut être approchée par une droite qui se raccorde avec la droite normalement consolidée (NC) à l'abscisse $\sigma_{p}^{\prime}$; sa pente est inférieure à $\left(\operatorname{tg} \varphi^{\prime}\right)_{N C}$ et présente un terme de cohésion $\mathrm{c}^{\prime}$.

- Pour les sols indurés, la cohésion domine ; la cohésion c' est généralement élevée et très dispersée.

Lorsqu'une cohésion effective non nulle est utilisée dans les calculs, il faut s'assurer qu'elle sera disponible pour toutes les situations (court terme/long terme, passage éventuel à la résistance résiduelle, altération).

\section{5.}

\section{Enrochements}

Pour les enrochements, on adopte le plus souvent une courbe d'allure parabolique d'axe horizontal $\tau=\mathrm{A} . \sigma^{\mathrm{B}}$ (De Mello) (Fig. 2.5).

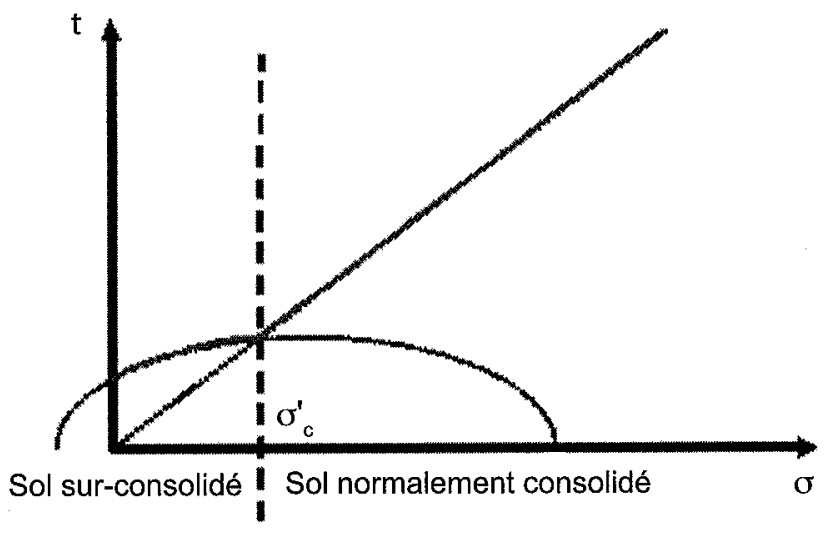

16. 21. Courbe intrinsèque pour un sol fin.

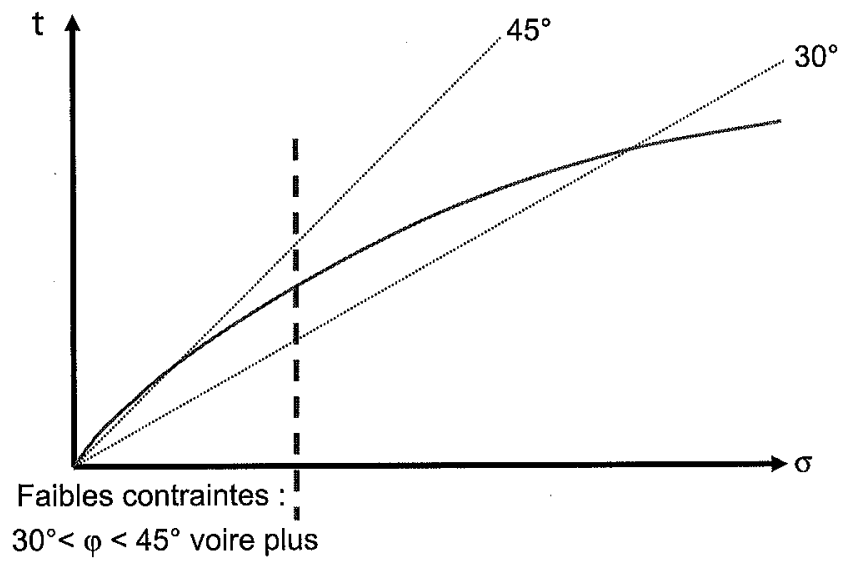

HG 2.5. Courbe intrinsèque parabolique pour un enrochement.

\section{5:}

\section{Rocher de fondation}

Pour les fondations rocheuses, on peut utiliser la relation de Hoek et Brown, qui définit un matériau homogène équivalent.

$$
\sigma_{1}=\sigma_{3}+\sigma_{c}\left(m_{b} \frac{\sigma_{3}}{\sigma_{c}}+s\right)^{a}
$$

avec :

$\sigma_{1}=$ contrainte principale majeure ;

$\sigma_{3}=$ contrainte principale mineure;

$\sigma_{c}^{3}=$ résistance à la compression uniaxiale de la roche intacte.

Pour l'étude d'un joint rocheux en fondation, on peut utiliser la relation de Barton, qui s'écrit :

$$
\tau=\sigma_{n} \tan \left(\varphi_{b}+J R C \log _{10}\left(\frac{J C S}{\sigma_{n}}\right)\right)
$$

avec :

$J R C$ le coefficient de rugosité ; 
JCS la résistance à la compression de la paroi, en $\mathrm{MPa}$;

$\varphi_{b} \quad$ l'angle de frottement de la paroi des joints ;

$\sigma_{\mathrm{n}} \quad$ la contrainte normale au joint, en $\mathrm{MPa}$;

$\tau$ la contrainte de cisaillement, en MPa.

Ces lois ne sont souvent pas directement implémentées dans les logiciels. Il est cependant possible la plupart du temps d'implémenter un critère de rupture point par point.

Pour utiliser une loi de Mohr-Coulomb représentative, il faut rechercher une droite équivalente à la courbe parabolique, dans la gamme de contraintes normales prévues, selon le cas, dans le remblai, au contact avec la fondation ou dans la fondation. Une illustration est donnée à la figure 2.6. La pente $\tan \left(\varphi_{\text {fond }}\right)$ et l'ordonnée à l'origine $\mathrm{C}_{\text {fond }}$ varient en sens inverse quand on parcourt la gamme de contraintes considérée. Les paramètres $\mathrm{C}_{\text {fond }}$ et $\tan \left(\varphi_{\text {fond }}\right)$ peuvent être estimés par la sécante passant par les deux points correspondant à la gamme de contraintes considérée.

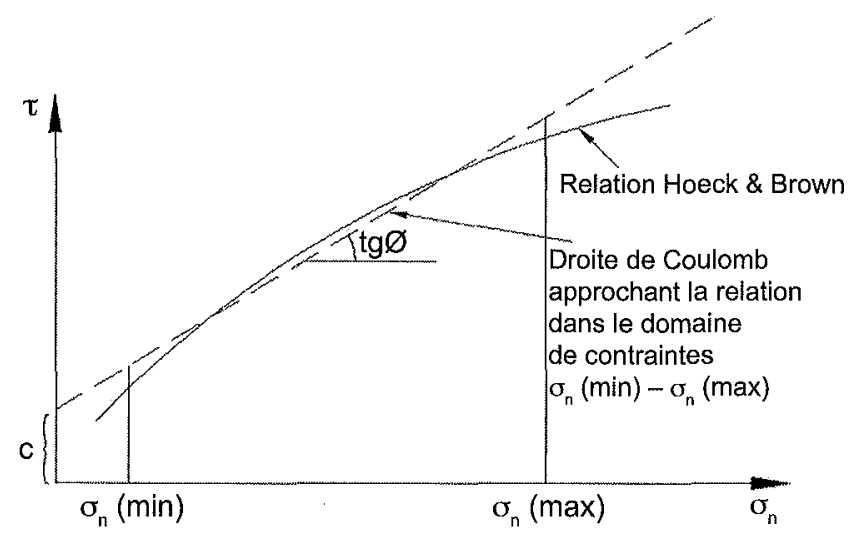

16. 26 Droite de Coulomb approchant la relation de Hoek et Brown pour déterminer $\mathrm{c}_{\text {fond }}$ et $\varphi_{\text {fond }}$.

\section{4.}

Valeur caractéristique des propriétés géotechniques

\subsection{7. \\ Généralités}

Les principes pour la détermination de la valeur caractéristique d'un paramètre géotechnique sont précisés dans la norme EN 1997-1 : 2004, autrement dénommée Eurocode 7 ( $\$ 2.4$ Dimensionnement géotechnique). Ces principes s'appliquent tout particulièrement aux remblais et les plus significatifs sont rappelés ci-après :

1) Le choix des valeurs caractéristiques des paramètres géotechniques doit s'appuyer sur les valeurs mesurées et les valeurs dérivées des essais en place et en laboratoire, complétées par les enseignements de l'expérience.

2) La valeur caractéristique d'un paramètre géotechnique doit être une estimation prudente de la valeur qui influence l'occurrence de l'état limite.
3) Lorsque l'on détermine les valeurs caractéristiques de $c^{\prime}$ et $\varphi^{\prime}$, on doit tenir compte du fait que la variance de $c^{\prime}$ est plus grande que celle de $\tan \varphi^{\prime}$.

4) Le choix des valeurs caractéristiques des paramètres géotechniques doit tenir compte des points suivants :

- l'existence d'informations préalables, [...] telles que des données de projets antérieurs ;

- la variabilité des valeurs mesurées des propriétés [...];

- le volume des investigations menées en place et en laboratoire ;

- le type et le nombre d'échantillons ;

- les dimensions de la zone du terrain qui gouverne le comportement de l'ouvrage géotechnique pour l'état limite considéré ;

- la capacité de l'ouvrage géotechnique à transférer les charges des zones faibles aux zones plus résistantes du terrain.

5) La zone du terrain qui gouverne le comportement d'un ouvrage géotechnique à un état limite ultime est en général beaucoup plus grande qu'une éprouvette de laboratoire ou que la zone affectée par un essai en place. Par conséquent, la valeur qui gouverne l'état limite est souvent une valeur moyenne d'un intervalle de valeurs couvrant une grande surface ou un grand volume de terrain. Il est recommandé que la valeur caractéristique soit une estimation prudente de cette valeur moyenne.

6) Si l'on utilise des méthodes statistiques, il convient que la valeur caractéristique soit déterminée de façon à ce que la probabilité calculée d'une valeur plus défavorable qui gouverne l'occurrence de l'état limite étudié ne dépasse pas $5 \%$.

Note : de ce point de vue, une estimation prudente de la valeur moyenne consiste à choisir la valeur moyenne d'un ensemble limité de valeurs du paramètre géotechnique avec un niveau de confiance de $95 \%$; par rapport à une rupture locale, une estimation prudente de la valeur la plus faible est un fractile à $5 \%$.

7) Lorsque l'on utilise des tables standardisées de valeurs caractéristiques liées aux paramètres de reconnaissance des sols, on doit choisir comme valeur caractéristique une estimation très prudente.

L'évaluation des résultats d'essais par des méthodes statistiques ne peut être réalisée que lorsque les données statistiques proviennent de populations identifiées suffisamment homogènes et qu'un nombre suffisant d'observations est disponible. Pour cela, il convient de prendre en compte la variabilité spatiale des paramètres, la dispersion des données d'essais et l'incertitude statistique associée au nombre d'essais.

Dans le domaine des ouvrages hydrauliques, l'utilisation des statistiques n'est que rarement possible et n'est pas toujours pertinente. L'estimation prudente fait alors appel au jugement de l'expert, à partir des résultats d'essais disponibles ou à partir de valeurs guides issues de la littérature. La valeur caractéristique correspond alors à une estimation experte prudente de la valeur de la résistance du matériau, responsable de l'apparition des états limites. 


\section{1.}

\section{Évaluation de la valeur caractéristique d'une propriété géotechnique}

La détermination des valeurs caractéristiques pour la justification de la stabilité d'un ouvrage appartient au concepteur. Pour cela, il s'appuie :

- sur le rapport établi par le géologue ;

- sur le rapport établi par le bureau de géotechnique (mission G11 et/ou G12), ce rapport devant présenter a minima une proposition de modèles géologique et géotechnique et des valeurs caractéristiques.

Cette démarche conduit le concepteur à intervenir dans le déroulement des missions géotechniques :

1) le concepteur définit les grandeurs à déterminer en regard des particularités de son projet ;

2) le bureau de géotechnique propose les méthodes de reconnaissances et de mesures les mieux adaptées, ce programme est discuté avec le concepteur ;

3) le bureau de géotechnique réalise les reconnaissances et mesures, et fournit un rapport interprété qui propose les valeurs caractéristiques à retenir pour le projet ;

4) le concepteur valide ou modifie les valeurs proposées pour son projet.

La dernière étape est nécessaire car :

- lorsque les valeurs caractéristiques sont obtenues par différentes méthodes, l'une ou l'autre des méthodes peut être plus représentative ;

- il n'y a pas de relation simple entre facteur de sécurité et probabilité de rupture : la prudence requise pour l'estimation de la valeur caractéristique dépend du projet.

Cette démarche montre que l'intégration d'une compétence géotechnique au sein même de l'équipe de conception est une nécessité absolue. Le concepteur et/ou le géotechnicien doit toujours faire le lien avec l'étude géologique.

Le choix des valeurs caractéristiques des propriétés géotechniques adoptées dans les notes de calcul doit être justifié de façon minutieuse et motivée. Ce choix doit s'appuyer sur trois catégories d'informations :

- une campagne de reconnaissances géologique et géotechnique, comprenant un nombre représentatif d'essais d'identification et des essais mécaniques réalisés selon les normes en vigueur ou selon des modes opératoires spécifiques. On distingue :

- les valeurs issues directement des essais,

- les valeurs résultant de calculs établis à partir des valeurs des essais et employant les équations et la théorie de la mécanique des sols et des roches, mais aussi les résultats issus de modèles empiriques et/ou de corrélations. Ces valeurs, bien entendu, présentent une incertitude plus élevée et il convient de se référer aux conditions d'évaluation de ces relations ;

- le retour d'expérience du géotechnicien issu de matériaux analogues ou de sites voisins. Ce retour d'expérience doit être détaillé et référencé dans les notes de calculs ;
- la connaissance des valeurs des paramètres géotechniques pour le type de matériaux, issues de la littérature. Les références bibliographiques utilisées dans les notes de calculs doivent être citées.

L'hétérogénéité des formations géologiques et géotechniques est appréciée à deux échelles d'observation :

- l'hétérogénéité (c à l'échelle des formations » est modélisée par la définition d'une succession des couches considérées comme étant homogènes, à l'intérieur desquelles les valeurs des différentes propriétés pourront être retenue avec la possibilité de les associer à une loi de variation fonction de la profondeur ;

- l’hétérogénéité ( à l'échelle de l'échantillon ») où les variations des propriétés sont prises en compte dans l'évaluation des propriétés elles-mêmes (anisotropie, etc.).

Si l'on dispose de données abondantes sur une zone de sol homogène gouvernant l'état limite étudié, on pourra, le cas échéant, mettre en œuvre des méthodes statistiques pour déterminer les valeurs caractéristiques. Les principes des méthodes statistiques sont rappelés dans l'Eurocode 7 et sont illustrés dans le guide du ministère de l'Équipement-CETMEF intitulé Recommandations pour le calcul aux états limites des aquatique : ROSA 2000.

Il est recommandé de procéder à des calculs de sensibilité portant sur les paramètres-clés. Cela vaut en particulier pour les paramètres difficilement accessibles de la résistance au cisaillement de la fondation. Cette démarche permet de mettre en évidence les paramètres prépondérants et de tenir compte des effets de seuil. Les études de sensibilité réalisées doivent figurer dans les notes de calculs de stabilité.

Le géotechnicien établit le rapport géotechnique à partir des reconnaissances et essais effectués et de l'expérience locale qu'il peut avoir des terrains, expérience acquise en particulier lors d'études antérieures. De plus, dans le cas où plusieurs campagnes successives de reconnaissance sont effectuées, les propositions de valeurs caractéristiques de paramètres peuvent évoluer suite à l'amélioration des connaissances.

La qualité de la détermination des paramètres géotechniques dépendent de :

- la qualité de la reconnaissance géologique du site, qui permet notamment d'orienter la campagne géotechnique ;

- la complétude de la campagne géotechnique tant dans la réalisation des essais que dans l'analyse des résultats ;

- l'intervention du géotechnicien aux différentes étapes du projet ainsi que son implication dans la conception afin d'en intégrer les contraintes.

Dans une démarche d'autocontrôle et de validation, il apparaît indispensable de vérifier que les ordres de grandeur obtenus se situent dans les plages de variation connues pour les sols de nature similaire. Si d'importants écarts sont constatés, on examine la qualité 
des essais et, le cas échéant, on s'interroge sur la représentativité du modèle géologique (et donc de la pertinence du modèle géotechnique). Il doit être envisagé d'engager des reconnaissances complémentaires afin de lever les ambiguïtés.

Les caractéristiques sont associées à des couches de sols homogènes définies en fonction du modèle géométrique. On remarque donc à ce niveau la difficulté de définir des valeurs caractéristiques dans le cas de sols très hétérogènes conduisant à une forte dispersion des mesures.

Les propriétés des sols sont évaluées en cohérence avec les hypothèses du modèle hydraulique (niveaux piézométriques). Le couplage géotechnique-hydraulique a par exemple une importance certaine dans l'appréciation des résistances géomécaniques influencées par la teneur en eau et le degré de saturation.

Nous donnons ci-après des exemples de démarche pour la détermination de valeurs caractéristiques de propriétés de matériaux.

- Exemple 1 : valeur caractéristique du poids volumique

Les approches statistiques sont pertinentes compte tenu du nombre important de mesures.

- Exemple 2 : valeurs caractéristiques des résistances au cisaillement d'un sol pour construction d'un remblai homogène

Les essais triaxiaux consolidés drainés (ou consolidés non drainés avec mesure de la pression interstitielle) sont les essais courants de laboratoire les plus fiables pour déterminer le critère de rupture, sous réserve d'éviter les biais suivants :

- vitesse de cisaillement trop élevée ;

- contre-pression trop faible ;

- diamètre de l'éprouvette insuffisant par rapport au diamètre maximal des grains ;

- contrainte de confinement non représentative de celle du site.

Ces biais étant évités, toute éprouvette correctement écrasée fournit un point du critère de rupture. En supposant homogène l'origine des éprouvettes, on peut alors reporter l'ensemble des points sur un même graphique dans le plan de Mohr.

Si le critère est linéaire, on peut tracer la droite de corrélation ainsi que son intervalle de confiance à $9 \%$. On retient alors la droite qui s'ajuste sur la limite inférieure de cet intervalle de confiance (Fig. 2.7.a).

Si le critère est non linéaire, on reporte les points dans le plan de Mohr en échelles logarithmiques et on procède de la même façon que ci-dessus (Fig. 2.7.b). Dans ce cas, l'écart entre la droite caractéristique et la droite d'ajustement donne directement le coefficient partiel $\gamma=10^{\varepsilon}$.

- Exemple 3 : valeurs caractéristiques des résistances au cisaillement d'une fondation hétérogène en matériaux meubles

Les essais triaxiaux sur les matériaux de la fondation sont généralement moins nombreux et plus dispersés

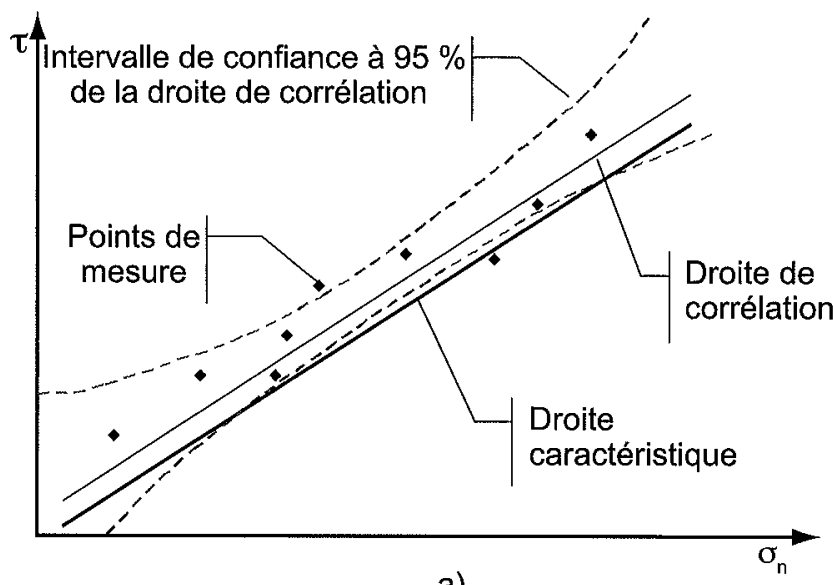

a)

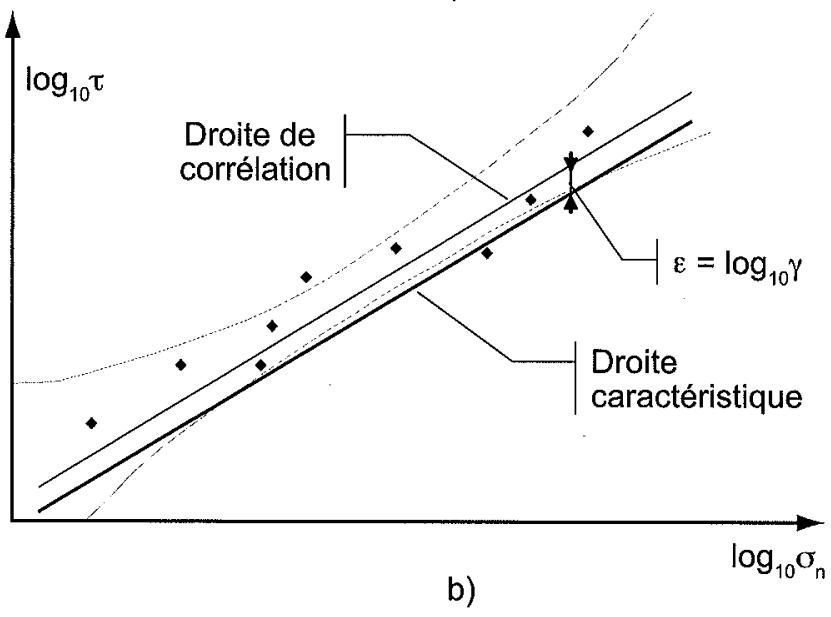

20. Interprétation d'une série d'essais triaxiaux réalisés sur un sol de remblai homogène (a) en échelle arithmétique et (b) en échelle logarithmique.

que ceux réalisés sur les matériaux du remblai. Il est peu probable que les matériaux soient homogènes car les échantillons peuvent provenir de couches différentes ou parce qu'il existe des zones plus faibles qui ont pu être découvertes par une ou quelques éprouvettes seulement. En l'absence de nouveaux sondages de confirmation, il convient d'interpréter les résultats disponibles avec la plus grande prudence, par exemple en considérant une cohésion effective nulle, sur le point le plus faible (Fig. 2.8).

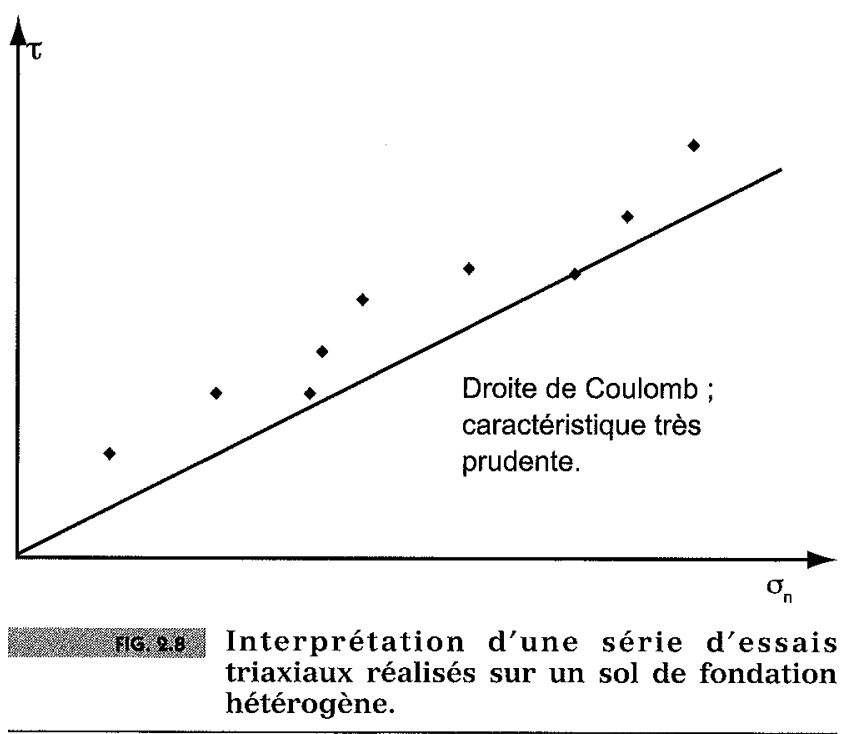




\section{1.2}

\section{Variabilité des données}

On peut distinguer plusieurs sources d'incertitudes affectant les données géotechniques et pouvant conduire à une certaine dispersion des valeurs :

- les incertitudes liées à la nature même des matériaux naturels:

- variabilité spatiale : formations hétérogènes,

- variabilité temporelle : caractéristiques géotechniques variables dans le temps du fait de l'historique de leur genèse et de leur environnement ;

- les incertitudes liées à la démarche même de la détermination des paramètres :

- représentativité de l'échantillon en regard de la formation à modéliser, le nombre d'essais étant toujours limité,

- incidence du nombre d'échantillons sur la décision des valeurs à retenir pour une même formation ;

- les incertitudes liées à la collecte et l'interprétation des informations :

- liées à l'exécution proprement dite des essais et ce sur toute la chaîne d'acquisition qui englobe la qualité du prélèvement et la qualité de réalisation des essais (impacts matériel et humain), sachant que l'ensemble de ces tâches fait l'objet d'une normalisation,

- liées à la pertinence du choix des essais en regard du paramètre à appréhender et de l'adéquation du chemin de contrainte expérimental à la réalité,

- liées à l’interprétation des essais.

Ce constat doit conduire à garder un esprit critique sur les valeurs obtenues pour :

- apprécier leur représentativité ;

- éviter de prendre une anomalie géologique pour une dispersion de l'échantillonnage et déceler les valeurs aberrantes.

L'étude de la variabilité des propriétés doit être effectuée à l'échelle spatiale pertinente au regard de l'état limite considéré. La zone du remblai et/ou de la fondation qui gouverne le comportement d'un ouvrage géotechnique à un état limite est en général beaucoup plus grande qu'une éprouvette de laboratoire ou que la zone affectée par un essai en place. Par conséquent, la valeur qui gouverne l'état limite est souvent une valeur moyenne d'un ensemble de valeurs couvrant une grande surface ou un grand volume de terrain. La valeur caractéristique est une estimation prudente de cette valeur moyenne.

\section{(16)}

\section{Méthodologie pour déterminer le modèle géotechnique}

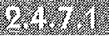

\section{Projet neuf : reconnaissance des sols de fondation}

La méthodologie pour la détermination des caractéristiques de base des sols peut être décomposée suivant les étapes principales suivantes :
1) Données initiales : collecte d'informations préalables

- visite de site ;

- collecte de données antérieures : sondages réalisés, analyse experte des essais disponibles;

- projets antérieurs ;

- étude géologique initiale.

\section{2) Campagne géotechnique :}

a) Établissement d'un programme de reconnaissances en regard de l'analyse des données recueillies et du projet :

- étendue de la zone à reconnaître ;

- nature des sondages et des essais. Les essais d'identification, les essais mécaniques et hydrauliques présentés au paragraphe 2.4.7.3 pour les remblais seront à réaliser. Les essais en laboratoire devront être réalisés sur des échantillons intacts, prélevés par sondages carottés. Si le prélèvement intact s'avère impossible, on s'attachera à reconstituer en laboratoire des échantillons présentant les mêmes caractéristiques de densité que celles rencontrées sur le terrain. Des essais in situ seront également employés pour caractériser le comportement mécanique des matériaux : pressiomètre, pénétromètre statique, piézocône ;

- implantation des sondages et des prélèvements;

- nombre d'essais (couverture du terrain). Le nombre d'essais à entreprendre peut être du même ordre de grandeur que ce qui est réalisé pour caractériser le remblai (cf. \$ 2.4.7.3) si l'épaisseur meuble est importante ;

- profondeur des reconnaissances.

b) Réalisation des essais, des sondages, des prélèvements, et présentation des résultats bruts. Il peut apparaître important d'engager un suivi de la campagne afin d'adapter son contenu au vu des premiers résultats bruts obtenus.

c) Évaluation de la représentativité des résultats bruts, de leur aptitude à être utilisés pour la détermination de valeurs caractéristiques et sélection des résultats d'essais validés.

\section{3) Géométrie du modèle géotechnique}

a) Identification des sols constitutifs de la fondation, détermination de la géométrie (stratigraphie plus ou moins complexe) et des conditions piézométriques :

- couches homogènes ;

- position du substratum porteur que l'on peut considérer comme la limite du modèle ;

- positions des horizons caractérisés par des comportements hydrauliques ou mécaniques singuliers : couches imperméables, couches drainantes, surface de rupture préexistante ;

- identification des conditions aux limites d'alimentation de nappes phréatiques, nappes captives...

b) Affectation de caractéristiques aux différents éléments du modèle ; détermination des caractéristiques retenues et appréciation de leur variabilité.

c) Contrôle : dans une démarche (c qualité », une analyse critique des valeurs est menée sur la base :

- d'expériences comparables ; 
- d'un examen des corrélations entre essais ;

- de la comparaison avec des valeurs « habituelles » (tableau de valeurs cibles).

4) Synthèse : proposition d'un modèle géotechnique combinant le ou les profils géométriques et les valeurs des différents paramètres servant à la justification. Il peut être nécessaire de rappeler le niveau d'incertitude des paramètres appréhendés.

\section{1:}

\section{Projet neuf : reconnaissance des fondations rocheuses}

Les fondations rocheuses des ouvrages hydrauliques en remblai présentent, dans la majeure partie des cas, des caractéristiques non dimensionnantes pour la justification des ouvrages, notamment pour les digues.

Dans le cas des fondations rocheuses que l'on peut considérer comme des milieux discontinus, les propriétés du massif sont dictées par :

- la masse rocheuse proprement dite, qui peut présenter des faiblesses liées à une altération homogène du massif rocheux ou une fracturation intense dans plusieurs directions de l'espace. En pratique, la masse rocheuse examinée dans les justifications est celle qui se situe à proximité du contact avec le corps du barrage. En effet dans le cas général, le rocher altéré et présentant les résistances mécaniques les plus faibles se situe en surface et la qualité mécanique de la fondation s'améliore avec la profondeur ;

- les discontinuités éventuellement présentes dans la masse rocheuse, aux faibles propriétés de résistance au cisaillement. Les justifications de stabilité peuvent concerner aussi bien des discontinuités situées en surface que des discontinuités localisées dans des zones de la fondation plus profondes. Le cas échéant, ces justifications sont dimensionnantes et non celles relatives à la masse rocheuse ;

- mais aussi des spécificités du massif rocheux en regard de la nature même de l'ouvrage constitué de remblai : singularités dans les calcaires (karst...).

On distingue les cas singuliers suivants :

- les matériaux rocheux altérés ou très fortement fracturés, qui peuvent être assimilés à des formations meubles ;

- les cas des fondations rocheuses évolutives et/ou singulières :

- les roches solubles : gypse...

- les roches présentant une structure géologique singulière,

- un contact géologique ayant un impact sur les écoulements et facilitant, par exemple, les résurgences,

- les structures lithologiques présentant des plans de faiblesse,

- des concentrations de circulation d'eau (karst...) conduisant à des conditions aux limites singulières mais préjudiciables à la tenue de l'ouvrage.
On cherche à apprécier l'état de contrainte résultant de la présence de l'ouvrage considéré et à vérifier si ces nouvelles sollicitations, qui peuvent avoir un impact sur le comportement du massif rocheux, restent acceptables.

Dans une première approche, on peut chercher à classifier le massif rocheux à l'aide de classifications développées pour les travaux souterrains et qui peuvent être adaptées aux fondations d'ouvrage : classification de Bieniawski, Barton...

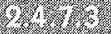

\section{Projet neuf : étude des matériaux d'emprunt}

Les matériaux constitutifs du remblai peuvent être issus de diverses sources:

- matériaux issus du projet et valorisés en remblai. L'exploitation de gisements dans l'emprise ou à proximité du futur réservoir oblige à mener une reconnaissance similaire à celle réalisée pour les fondations, complétée par un volet valorisation-réemploi des matériaux. La quantification des volumes conduit (selon les phases d'études) à étendre la prospection, à étudier le comportement de matériaux recomposés... Ces campagnes permettent plus facilement d'engager une approche statistique que les campagnes de reconnaissance de fondation;

- matériaux de carrière en exploitation. Dans ce cas, on a affaire à des matériaux plus ou moins normalisés dont l'homogénéité relative conduit à une moindre dispersion des caractéristiques.

Les essais à mener se répartissent en :

- essais d'identification : teneur en eau naturelle, granulométrie et sédimentométrie, limites d'Atterberg, essai au bleu de méthylène ;

- poids volumique des grains, poids volumique apparent...;

- essais de compactage des matériaux des zones d'emprunt : essai Proctor normal ;

- essais mécaniques : mesure de la résistance à la compression simple, résistance au cisaillement (à l'appareil triaxial pour les matériaux fins, à la boîte de Casagrande pour les matériaux grossiers), compressibilité à l'œdomètre ;

- essais hydrauliques : mesure de la perméabilité au moule Proctor d'un matériau compacté à l'OPN ou $95 \%$ de l'OPN à l'œdomètre ou au perméamètre ;

- essais de résistance à l'érosion : hole erosion test (HET), jet erosion test (JET).

Les modules d'élasticité et œdométriques sont déterminés à partir :

- des essais in situ : essais pressiométriques, essais au pénétromètre statique. Des corrélations ont été établies entre les propriétés pressiométriques (pression limite $p_{1}$, pression de fluage $p_{\mathrm{f}}$, module pressiométrique $\mathrm{E}_{\mathrm{M}}$ ), d'une part, et entre les propriétés pressiométriques et les propriétés pénétrométriques (résistance de pointe $q_{c}$ ), d'autre part (cf. tableaux en annexe 1). Soulignons que ce type de corrélations permet principalement de réaliser des recoupements entre les résultats obtenus par différents moyens d'investigation. Ils ne doivent pas conduire à se contenter de campagnes de reconnaissance trop sommaires pour justifier le dimensionnement d'un ouvrage ; 
- des essais de laboratoire : essais triaxiaux, essais œdométriques.

Le nombre d'essais de chaque type est à adapter au volume probable du remblai, à sa hauteur, au nombre de matériaux de différents types utilisés (cas des barrages zonés) et à la variabilité naturelle des matériaux étudiés.

Le guide du CFBR Petits barrages - Recommandations pour la conception, la réalisation et le suivi propose un programme minimal d'essais pour les matériaux d'emprunt de barrages en terre ne présentant pas de difficulté particulière, déterminé en fonction du volume de matériau à reconnaître (soit 1,5 à 2 fois le volume géométrique du remblai) :

- série d'essais d'identification (teneur en eau naturelle, granulométrie, sédimentométrie, limites d'Atterberg): un pour 5000 à $10000 \mathrm{~m}^{3}$ de matériaux à mettre en œuvre avec un minimum de cinc essais ;

- essais de compactage (Proctor Normal) : un pour 15000 à $25000 \mathrm{~m}^{3}$ avec un minimum de cinq essais ;

- essais de comportement mécanique et hydraulique (cisaillement triaxial, compressibilité à l'œdomètre, mesure de la perméabilité) : un pour 30000 à $50000 \mathrm{~m}^{3}$ avec un minimum de trois essais (mais aucun essai de ce type n'est préconisé lorsque $\mathrm{H}^{2} \mathrm{~V}^{1 / 2}<5$ ).

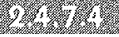

\section{Ouvrage existant}

Dans le cas des barrages les plus importants et instrumentés, la méthodologie pour la détermination du modèle géotechnique d'un ouvrage existant est différente de celle d'un ouvrage en projet car elle utilise toutes les connaissances acquises lors la surveillance du barrage, notamment les résultats des mesures d'auscultation.

Les principes exposés précédemment aux $\$ 2.3 .7 .1$ et 2.3.7.3 restent valables. Les valeurs caractéristiques des propriétés de résistance d'un ouvrage existant reposent sur les reconnaissances géotechniques et géophysiques réalisées en phase de conception et sur les essais réalisés lors de l'exécution des travaux, complétés généralement par de nouvelles investigations réalisées spécifiquement pour caractériser la fondation et le remblai en place. On privilégie les reconnaissances non destructives : essais pénétrométriques, pressiométriques, méthodes géophysiques, sondages carottés avec prélèvement d'échantillons intacts assortis d'essais de laboratoire.

Pour les ouvrages plus modestes, avec peu ou pas de données géotechniques disponibles ni de mesures d'auscultation, on peut se retrouver dans une situation proche d'une situation de projet (hormis l'aspect recherche de matériau qui sera limité à celle des matériaux de confortement). La campagne à mener dans ce cas devra être relativement complète, avec néanmoins une orientation donnée par la pathologie constatée ou les interrogations qui ont conduit à vouloir modéliser cet ouvrage.

\section{3)}

\section{Recommandations relatives aux essais}

En guise de principe général, on peut souligner que, dans tous les cas, il faut s'assurer que les conditions d'essai soient choisies de telle sorte que les contraintes à la rupture des échantillons couvrent la gamme des valeurs caractéristiques des contraintes auxquelles seront supposés être soumis les ouvrages projetés (contraintes d'ELU, voire contraintes d'ELS) dans le sol en place et à la profondeur correspondant à celle du prélèvement.

En outre, on s'attachera à mener les essais jusqu'à la profondeur à partir de laquelle l'influence du projet devient négligeable.

\section{3.}

\section{Précautions pour des essais de qualité}

Les prélèvements, le conditionnement des échantillons, les essais géotechniques en place et de laboratoire, requièrent une très bonne maîtrise de leur technique. Quelques problèmes classiques pouvant invalider les résultats sont répertoriés ci-dessous.

15ALACA 21I Précautions pour des essais de qualité (tableau extrait de ROSA 2000).

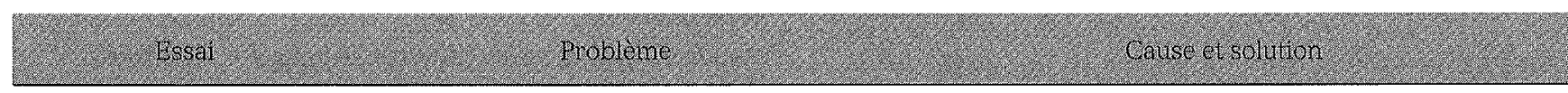

Essais en place

Pressiomètre

Pénétromètre

Scissomètre
Remaniement des parois du forage

Faux refus

Mobilisation incontrôlable du frottement
Demander un forage à la boue bentonique épaisse

Frottement des tiges, présence de blocs

Sol non purement cohérent (essai non adapté)

Essais de laboratoire

Remaniement des échantillons

A contrôler très sérieusement : conditionnement des carottes, transport, présence contradictoire du géotechnicien à l'ouverture au laboratoire 
Il faut y ajouter le problème de la représentativité des essais de laboratoire sur les matériaux grossiers, pour lesquels on est souvent amené à écrêter le matériau de façon à respecter le $\mathrm{D}_{\max }$ en fonction de la taille de l'éprouvette, sachant qu'au-delà d'une certaine proportion d'écrêtement (de l'ordre de 25 à $30 \%$ ), l'essai perd de sa représentativité. Il convient alors de recourir à des appareils triaxiaux ou à des boîtes de cisaillement de grandes dimensions.

De très nombreux essais en laboratoire ou in situ font l'objet d'une norme. Le site de l'AFNOR permet d'obtenir une liste à jour des documents de référence en la matière.

\subsection{Th}

\section{Mesure de la résistance au cisaillement à court terme}

Ces essais s'effectuant sur des sols fins, le mode opératoire des essais est différent selon l'état de saturation initial de l'échantillon, et l'état de saturation du sol en place après les travaux. En outre, l'importante variabilité des résultats pourra conduire à augmenter le nombre des essais afin de mieux cerner cette caractéristique.

Les paramètres de résistance au cisaillement non drainé sont obtenus :

- par des essais de laboratoire : l'essai triaxial de type UU (non consolidé, non drainé) ou de type CU (consolidé, non drainé) sur des éprouvettes de dimensions normalisées. L'essai rapide à la boîte de cisaillement est déconseillé, même dans les sols argileux très peu perméables, en raison du drainage partiel inévitable ;

- par des essais in situ : l'essai scissométrique ;

- à partir de corrélations qui les relient à l'essai pressiométrique (corrélation $\mathrm{C}_{\mathrm{u}}-\mathrm{P}_{\mathrm{LM}}$ ), à l'essai de pénétration statique (corrélation $\mathrm{C}_{u}-\mathrm{q}_{\mathrm{c}}$ ) ou à l'essai de pénétration au carottier SPT (corrélation $\mathrm{C}_{\mathrm{u}}-\mathrm{N}_{30}$ ) :

- $\mathrm{C}_{\mathrm{u}}=\left(\mathrm{P}_{\mathrm{LM}}-\mathrm{p}_{\mathrm{o}}\right) / 5,5, \mathrm{P}_{\mathrm{LM}}$ étant la pression limite mesurée lors de l'essai pressiométrique et $\mathrm{p}_{0}$ étant la pression horizontale des terres au repos;

- $\mathrm{c}_{\mathrm{u}}=\left(\mathrm{q}_{\mathrm{c}}-\mathrm{q}_{\mathrm{o}}\right) / 17, \mathrm{q}_{\mathrm{c}}$ étant la résistance de pointe à l'essai pénétrométrique et $q_{0}$ étant la contrainte verticale au niveau de l'essai ;

- $\mathrm{c}_{\mathrm{u}}(\mathrm{kPa})=15 \mathrm{~N}_{30^{\prime}} \mathrm{N}_{30}$ étant le nombre de coups nécessaires pour enfoncer le carottier SPT de $30 \mathrm{~cm}$ après l'avoir initialement enfoncé de $15 \mathrm{~cm}$.

Ces relations ne donnent que des ordres de grandeur, en particulier les deux dernières.

Les éléments présentés ci-dessus permettent d'évaluer la résistance au cisaillement à court terme qui pourra être utilisée dans un calcul en contraintes totales. Dans tous les cas, la démarche sera doublée d'un calcul en contraintes effectives à l'aide de $c^{\prime}, \varphi^{\prime}$ et $u$ (pression interstitielle).

\section{3.}

Mesure de la résistance au cisaillement en conditions drainées : sols granulaires et sols fins à long terme

La détermination de la résistance au cisaillement drainé des sols fins est toujours délicate en raison de la difficulté à réaliser correctement le drainage de l'échantillon. A titre indicatif, pour obtenir des résultats de fiabilité comparable, il faut 2 à 3 fois plus d'éprouvettes pour un matériau raide et fissuré que pour un sol mou.

Pour les sols fins surconsolidés qui présentent de la cohésion, il est donc conseillé d'ajouter quelques essais sous fortes contraintes de façon à déterminer l'angle de frottement du matériau normalement consolidé.

Les paramètres de résistance au cisaillement drainé des sols sont obtenus:

- à partir d'essai de laboratoire sur des éprouvettes saturées:

- essai triaxial CD (consolidé, drainé),

- essai triaxial CU + u (consolidé, non drainé, avec mesure de la pression interstitielle),

- en l'absence d'essai triaxial, essai de cisaillement direct (boîte de cisaillement). Les essais de cisaillement direct à la boîte, de type drainé (essais lents), sont toutefois déconseillés pour les sols fins en raison de la difficulté à obtenir un drainage complet ;

- à partir de corrélations reliant $\varphi^{\prime}$ à l'état de compacité du sol et des données granulométriques, ou aux propriétés mesurées dans les essais en place, principalement le nombre $\mathrm{N}_{30}$ de coups de mouton mesuré dans l'essai de pénétration au carottier SPT (corrélation $\varphi^{\prime}-N_{30}$ ), la résistance de pointe mesurée dans l'essai de pénétration statique (corrélation $\varphi^{\prime}-q_{c}$ ) et la pression limite mesurée lors de l'essai pressiométrique Ménard (corrélation $\varphi^{\prime}-\mathrm{P}_{\mathrm{LM}}$ ).

Ces corrélations sont indiquées à titre informatif en annexe 1 du fait qu'elles sont en général peu précises et s'appliquent dans des domaines de sols particuliers ; ces corrélations ont le plus généralement été établies pour des sols pulvérulents $\left(\mathrm{C}^{\prime}=0\right)$.

\section{9. $\%$.}

\section{Mesure de la perméabilité}

Les valeurs de perméabilité saturée sont obtenues par de nombreux essais (cf. tableau 2.III). Leur choix dépend du phénomène étudié. Dans le tableau 2.III, les huit premières lignes concernent des essais in situ tandis que les deux dernières concernent des essais en laboratoire.

Les essais les plus représentatifs pour l'évaluation de la perméabilité en grand sont les essais in situ de grand rayon d'action.

\section{1.}

\section{Mesure du taux de développement de pressions interstitielles de fin de construction}

Ce coefficient est évalué par l'une des trois méthodes suivantes :

1) A partir d'une étude paramétrique d'essais triaxiaux de laboratoire où l'argile a été humidifiée et compactée conformément aux spécifications, Bishop (1954) propose que les pressions interstitielles soient calculées en utilisant les coefficients A et B de Skempton (1954). Tout incrément de pression $(\Delta u)$ sur un chemin de contraintes triaxial est la somme d'un incrément provoqué par la contrainte isotrope $B(\Delta \sigma 3)$ et d'un incrément dû au déviateur $\operatorname{BA}\left(\Delta \sigma_{1}-\Delta \sigma_{3}\right)$. 


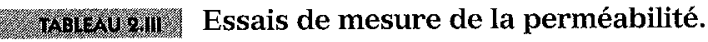

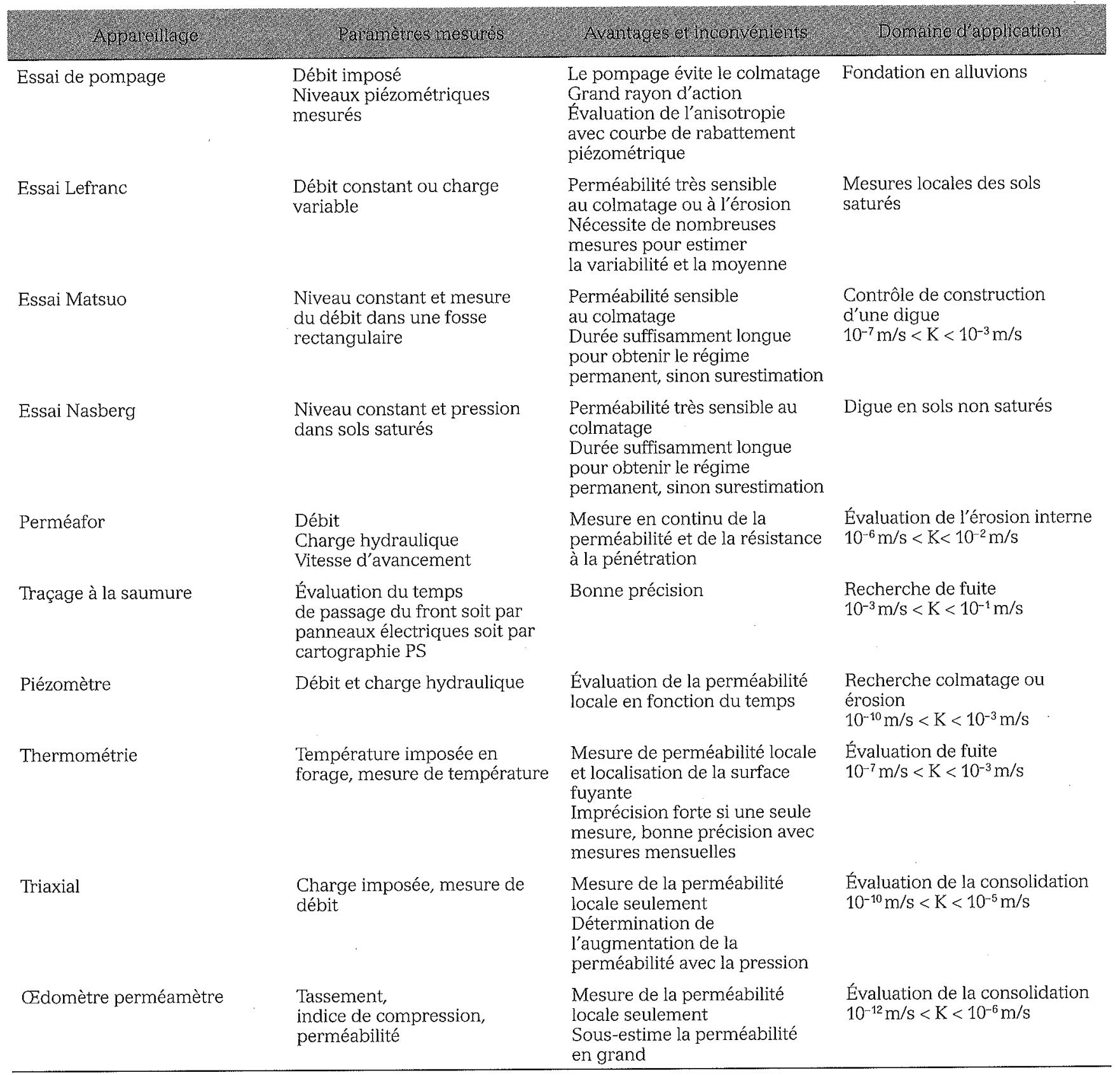

$$
(\Delta \mathrm{u})=\mathrm{B}\left[\Delta \sigma_{3}+\mathrm{A}\left(\Delta \sigma_{1}-\Delta \sigma_{3}\right)\right]
$$

Il est alors possible d'exprimer l'augmentation de pression sous l'augmentation du poids des couches supérieures :

$$
\bar{B}=\frac{\Delta u}{\Delta \sigma 1}=B \cdot A\left[1+\frac{\Delta \sigma_{3}}{\Delta \sigma_{1}}\left(\frac{1}{A}-1\right)\right]
$$

avec :

u pression interstitielle ;

$\sigma_{1}$ contrainte principale majeure, assimilée à $\gamma \mathrm{h}$;

$\gamma$ poids volumique du sol au-dessus du point considéré ;

h hauteur de la colonne de sol au-dessus du point considéré.

Cette méthode est lourde sur le plan des reconnaissances. Elle est nécessaire pour déterminer la pres- sion interstitielle en tout point d'un remblai homogène. Le paramètre A mesure l'influence du déviateur sur la pression. Cette influence est forte quand le sol est proche de la rupture. A augmente quand le coefficient de sécurité et le degré de surconsolidation diminuent. Sous forte contrainte, ou après faible compactage, il tend vers 1 (et peut même dépasser 1).

2) Une deuxième méthode est la méthode USBR. Initiée par Bruggeman (1939), elle a été promue par Hilf (1948), en supposant l'absence de drainage, en combinant la loi de Mariotte pour la compressibilité de l'air avec celle de Henry pour exprimer la solubilité de l'air dans l'eau avec celle du sol mesurée au consolidomètre ou à l'œdomètre. L'expression de la pression interstitielle est supposée égale à celle de la pression de l'air.

$$
p=-\frac{p_{a} \cdot \Delta}{V_{a}+h V_{W}-\Delta}=\frac{p_{a} \Delta}{n\left(1-S_{r}+h S_{r}\right)-\Delta}
$$


avec :

p la pression interstitielle après chargement moins la pression atmosphérique ;

$\mathrm{p}_{\mathrm{a}}$ la pression atmosphérique;

$\Delta$ la variation relative de volume due à la consolidation (déformation volumique) ;

$V_{a}$ volume d'air libre après compactage exprimé en pourcentage du volume de sol ;

$V_{W}$ volume d'eau après compactage exprimé en pourcentage du volume de sol ;

h la constante de Henry $\left(0,0198\right.$ à $\left.20^{\circ} \mathrm{C}\right)$;

n porosité ;

$S_{r}$ degré de saturation.

Cette méthode est sécuritaire pour évaluer les pressions au centre du remblai : sa marge de sécurité réside d'une part dans la succion initiale négligée ou plus généralement dans l'écart entre pression d'air et pression d'eau et d'autre part dans la dissipation de la pression supposée nulle par absence de drainage. Elle est adaptée aux noyaux de barrages zonés, car elle suppose que seuls les tassements verticaux interviennent pendant la construction et que le rapport entre les contraintes effectives verticale et horizontale est inférieur à 2. En revanche, cette méthode peut sousestimer les pressions sur les bords d'un remblai homogène, surtout si sa teneur en eau dépasse l'optimum de plus d'un point. Il convient alors de se reporter à la première méthode.

3) Enfin, l'adoption de coefficients $r_{u^{\prime}}$ mesurés lors de la construction de barrages où l'argile est mise en place dans des conditions similaires, est justifiée par une forte expérience.

Le risque d'erreur d'estimation du coefficient $r_{u}$ peut justifier une approche plus générale qui intègre, par une méthode numérique, la loi de comportement du sol et la couple avec l'écoulement de l'eau dans chaque élément du maillage. Une telle approche est possible avec certains logiciels.

\section{3}

\section{Modèle hydraulique interne}

\section{1.}

\section{Les objectifs du modèle hydraulique interne}

Il s'agit de fournir le champ des pressions interstitielles et le champ des gradients hydrauliques au sein de l'ouvrage en fonction des conditions aux limites hydrauliques appliquées.

\section{9}

\section{Méthode pour la réalisation d'un calcul hydraulique}

Le préalable à la réalisation du modèle hydraulique comprend les étapes suivantes :

- élaboration du modèle géométrique ;

- élaboration du modèle géologique et hydrogéologique ;

- élaboration du modèle géotechnique.
A partir des trois modèles précédents, il convient de :

- caractériser les données d'entrée du modèle hydraulique interne. Il existe deux types de données :

- les propriétés intrinsèques à la distribution des tailles de pores de chaque couche,

- et les conditions aux limites ;

- choisir la modélisation adaptée à la situation de chargement hydraulique ;

- analyser les résultats de la situation de chargement étudiée : examen critique, étude de sensibilité ou vérification des erreurs à éviter.

\section{3., 3}

\section{Caractérisation des données d'entrée du modèle hydraulique}

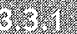

\section{Nature des données}

Le modèle hydraulique rassemble trois types de propriétés hydrauliques :

1) les propriétés intrinsèques liées à la distribution des pores du milieu poreux :

- porosité,

- perméabilité horizontale,

- perméabilité verticale,

- courbe hydrique 1 : succion fonction du degré de saturation,

- courbe hydrique 2 : perméabilité relative fonction du degré de saturation ;

2) les propriétés intrinsèques liées à la déformabilité du milieu poreux, utiles pour un calcul en conditions transitoires :

- modules de compressibilité,

- coefficient d'emmagasinement ;

3) les conditions aux limites des nappes :

- nappe amont,

- nappe aval,

- frontières imperméables,

- surfaces de suintement,

- sources,

- puits ou drains.

Parmi les données d'entrée, l'attention devra particulièrement être portée sur la détermination des perméabilités horizontales et verticales, en fondation et dans le remblai, et sur les conditions aux limites.

Les autres propriétés intrinsèques sont relatives aux écoulements en milieu non saturé et/ou transitoires. Dans la majorité des cas, elles influencent peu les résultats pour la détermination des pressions inters titielles de la situation, ( régime permanent ) et des valeurs par défaut (proposées souvent par les logiciels) pourront être adoptées. 


\section{Conditions aux limites}

Les conditions aux limites sont des hypothèses très importantes. Elles conditionnent très fortement les résultats du modèle hydraulique, aussi doivent-elles être clairement définies.

L'interface du modèle avec la retenue est définie comme une frontière à potentiel constant. L'imposition du potentiel de la retenue sur le bord amont du modèle est la situation la plus pénalisante. Elle n'est pas systématique. En l'absence d'alimentation amont par nappe ou par source, il est possible d'adopter un débit nul sur le bord amont.

La nappe aval est imposée sur la frontière aval. Cette frontière doit être suffisamment éloignée de l'ouvrage pour éviter de sous-estimer les potentiels en fondation.

La base horizontale du modèle est généralement prise comme une ligne de courant, traversée par un débit nul. Elle devrait être appliquée sur une couche imperméable.

Les drains peuvent être modélisés par des points où le potentiel est imposé comme le potentiel aval plus une éventuelle perte de charge (entre le drain et la nappe aval).

Les sources peuvent être modélisées par des points où le débit est constant.

Il existe souvent une couche de colmatage sur le talus amont des digues ou un culot de sédiments en fond de retenue de barrage ; il convient de modéliser cette couche dans le modèle géométrique, plutôt que de fixer un débit nul ou fixé. Il convient aussi de prendre en compte les situations où l'érosion peut emporter ce colmatage.

\section{tes}

\section{Cas des ouvrages anciens : calage des données}

Dans les études de réhabilitation ou les diagnostics de sécurité d'ouvrages anciens, il est vivement recommandé de justifier la stabilité à partir d'une modélisation hydraulique. Cette modélisation doit permettre de retrouver et d'expliquer le comportement de l'ouvrage. A cette fin, le modèle doit être calé avec les séries chronologiques de mesures de pression interstitielle. Une erreur moyenne de $5 \%$ sur les pressions est acceptable, à défaut il convient d'encadrer les mesures par une modélisation par excès des piézomètres.

\section{4}

\section{Choix de la modélisation des différentes situations}

\section{3.}

\section{La construction}

La construction ne pose pas de réelles difficultés lorsque les matériaux constitutifs sont perméables. En revanche, elle est certainement l'époque la plus critique des barrages en argile. En effet, l'argile doit avoir une teneur en eau moyenne à forte pour assurer la plasticité nécessaire à l'étanchéité. Mais comme l'argile est très compressible, elle reportera les contraintes de la construction sur son eau interstitielle, qui montera en pression sous la charge du remblai. Cette génération de pression a entraîné plusieurs ruptures de remblai : Mondély en 1981, Mirgenbach en 1982 et Carsington en 1984. Dans les cas les plus extrêmes, la pression interstitielle atteint le poids des terres, annule la contrainte effective et crée une fracturation hydraulique sur une section horizontale : Mirgenbach en 1982.

La prédiction de cette génération de pression est donc une étape capitale du dimensionnement des barrages en argile ou en limon. Parmi les méthodes à employer, les plus simples sont celles utilisant le coefficient $r_{u}$ et les plus rigoureuses sont basées sur la méthode des éléments finis.

\section{1.}

\section{La mise en eau}

La mise en eau est à modéliser pour les ouvrages de grande hauteur. Trois méthodes sont possibles :

- la méthode la plus simple consiste à fixer la surface libre qui traverse le barrage en partant de la retenue jusqu'à la nappe aval et à supposer constant le potentiel à la verticale de la surface libre. Cette approche suppose que l'écoulement est seulement horizontal. Elle est peu utilisée pour cette situation : elle sous-estime généralement les pressions réelles en fondation ;

- une méthode un peu plus élaborée est basée sur l'utilisation de logiciels d'écoulement transitoire en hydraulique pure (sans calcul mécanique des déformations). En effet, l'objectif de cette phase est de prévoir le comportement lors de la mise en eau et de réagir en cas d'anomalie vis-à-vis de la prévision. La limitation de cette approche est l'absence de couplage hydraulique-mécanique. Certains logiciels peuvent faire varier la porosité avec la pression d'eau. Dans ce cas, le changement de la porosité n'est pas piloté par les contraintes, comme sur site, mais par la progression de l'écoulement. Le logiciel d'écoulement ne reproduit pas la montée des pressions causée par le chargement de la retenue. Ainsi, une partie des pressions à l'amont de l'axe du barrage peut être sous-estimée. L'erreur n'est pas acceptable pour les grands remblais homogènes en argile, elle est négligeable ou tolérable pour les barrages zonés ;

- le calcul le plus juste est le calcul couplé dans lequel les deux composants de la génération de pressions interstitielles, l'écoulement et le chargement, sont reproduits. Seuls les logiciels de mécanique adaptés à la géotechnique et à l'hydraulique, par exemple GEFDYN, ASTER et FLAC, ont ces fonctionnalités.

\section{$31 x^{2}$}

\section{Le régime permanent}

La méthode qui consiste à fixer la surface libre est souvent utilisée, car elle a le mérite de la simplicité. Cependant, elle a très souvent l'inconvénient de sousestimer les potentiels en fondation sous la recharge 
aval. Cette méthode est déconseillée lorsque la fondation possède au moins une couche plus perméable que la base du remblai et qu'elle ne possède pas d'écran d'étanchéité.

La méthode la plus fiable est l'utilisation d'un logiciel d'hydraulique pure. En régime permanent, la consolidation n'existe plus et les logiciels couplés ne sont plus nécessaires.

Une approche intermédiaire consiste à imposer le potentiel aux points où il a été calculé par une méthode graphique. La méthode graphique est applicable dans le cas d'un remblai homogène ou d'un noyau en argile entre deux recharges perméables. Un changement de variable est cependant nécessaire pour reproduire les effets de l'anisotropie.

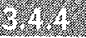

\section{La vidange}

La méthode la plus courante est la méthode de Bishop. Elle considère que la baisse de pression dans le barrage est obtenue par déchargement uniquement, ce qui suppose que la recharge amont est saturée et que l'eau n'a pas le temps de se drainer pendant la vidange. Cette méthode peut cependant sous-estimer les pressions, notamment dans les premières années d'exploitation des remblais homogènes en argile, époque où l'argile n'est pas encore saturée, et sous les masques amont en cas de drainage déficient (prise en compte du coefficient $r_{u}$ en vidange).

L'usage d'un logiciel d'écoulement transitoire en hydraulique pure est une méthode qui est souvent (mais pas toujours) sécuritaire, sans être la plus pénalisante.

L'usage d'un calcul couplé est rare, il n'est justifié que pour les grands ouvrages et les ouvrages complexes (fondation molle, recharge en matériau non drainant).

Une dernière méthode est possible, la méthode de Laplace. L'écoulement transitoire est remplacé par un écoulement permanent dont les potentiels amont sont donnés par la surface libre de la méthode de Bishop et les potentiels aval sont inchangés. Elle est proposée dans certains logiciels, tels que PLAXIS ; l'inconvénient est que son erreur est variable et difficile à évaluer.

Il existe donc une forte incertitude sur les résultats de la modélisation de la vidange, qui est prise en charge par le coefficient de modèle relatif à cette situation de projet.

\section{5}

\section{Le passage d'une crue}

La méthode la plus sécuritaire et la plus courante consiste à calculer un régime permanent dont la condition aux limites amont est la cote maximale de crue.

La méthode la plus réaliste est de modéliser la crue par un écoulement transitoire, en adoptant des perméabilités estimées par excès. Cette méthode engendre une petite erreur du fait de l'absence de surpres- sion causée par le chargement de la retenue; cette erreur est quasi nulle dans la recharge aval; sachant qu'il s'agit de vérifier la stabilité aval, cette erreur est négligeable et toujours tolérable.

- Cas particulier des digues fluviales

Pour les calculs des pressions interstitielles dans le cas des digues de protection contre les inondations en situation de crue, l'hypothèse du régime permanent est parfois très conservatrice et les données manquent en général pour faire un calcul réaliste en régime transitoire (il faut des courbes $k-S_{r}$ et $w-S_{r}$ : perméabilité et teneur en eau en fonction de la saturation). Par ailleurs, les résultats sont souvent dispersés pour ces calculs dès qu'ils font intervenir les caractéristiques mal connues en milieu non saturé.

Il conviendra donc de retenir des hypothèses raisonnablement conservatives sur les conditions aux limites et sur la position de la ligne de saturation, en fonction de la perméabilité des matériaux, de leur anisotropie, de la présence de singularités (en particulier horizontales) au sein de la digue et de la durée des crues. On prendra en compte le fait que la fondation est le plus souvent saturée dès le début de la crue.

\section{5}

\section{Analyse critique des résultats}

L'exposé des différentes méthodes montre qu'elles ont toutes des limitations. La méthode la plus juste, le calcul couplé, a pour inconvénient une lourdeur et un délai de mise en œuvre, qui parfois décourage l'ingénieur.

Aussi, l'ingénieur doit se souvenir que ces outils ne sont pas parfaits et qu'ils peuvent aboutir à des erreurs non acceptables. Il doit retenir que l'évaluation du modèle hydraulique est, dans la majorité des cas, la source principale d'erreur.

Avant de lancer les calculs, il importe qu'il analyse le choix de ses hypothèses et de ses outils afin de minimiser ce risque d'erreur.

Enfin, selon la perméabilité des matériaux, les phases liées à des régimes transitoires (en particulier construction et mise en eau) peuvent durer plus ou moins longtemps. Cette durée peut, dans certains cas, être de plusieurs années.

\section{4 \\ Justification de la stabilité des barrages en remblai}

\section{4 \\ Généralités}

La justification de la stabilité des barrages en remblai est conforme aux démarches semi-probabilistes aux états limites et examine successivement : les situations de projet, les actions, les résistances, les états limites et les critères de stabilité (voir introduction). 
Le cadre général de la définition des situations de projet et du calcul des actions est fixé dans la partie 1 (respectivement $\$ 1.1$ pour les situations de projet et $\S 1.2$ pour les actions). Les principes de détermination des valeurs de calcul des propriétés de résistance, notamment ceux relatifs à la valeur caractéristique, sont définis dans la partie 2 (§ 2.4.6). Le réseau d'écoulement et le régime des pressions interstitielles sont supposés connus pour le calcul de stabilité, en fonction des conditions aux limites hydrauliques appliquées, selon les principes présentés en partie 3 .

Cette partie s'attache à définir les différents états limites des barrages en remblai, les critères de stabilité, les coefficients partiels et de modèle associés (voir en introduction la démarche adoptée pour la détermination de ces coefficients) et les modes de justification.

\section{9}

\section{Les états limites}

Les états limites suivants doivent être considérés pour les barrages en remblai :

- l'état limite ultime de stabilité d'ensemble (glissement) ;

- l'état limite ultime de soulèvement hydraulique ;

- l'état limite ultime de défaut de portance ;

- l'état limite de service de tassement;

- l'état limite ultime de résistance à l'érosion interne ;

- l'état limite ultime de résistance à la surverse ;

- l'état limite de service de filtration.

Les états limites de résistance à l'érosion interne, à la surverse et de filtration seront traités dans une étape ultérieure, sachant que les suites du projet de recherche ERINOH (ERosion INterne des Ouvrages Hydrauliques) ont conduit à la mise en chantier de recommandations professionnelles destinées à proposer des critères de justification de ces états-limites. Pour les autres états limites, des conditions d'état limite sont décrites ci-après.

\section{$43^{3}$}

\section{Situations envisagées}

Sur la base du cadre général des situations de projet fixé en partie 1, les principales situations considérées pour les barrages en remblai sont données au tableau 4.I et servent de guide pour le concepteur.

\section{7}

\section{État limite ultime de stabilité d'ensemble (glissement)}

\section{4.t.}

\section{Préambule}

La stabilité d'ensemble et la résistance au glissement d'un barrage en remblai font intervenir des mécanismes complexes qui agissent sur un matériau hétérogène. Il existe des méthodes utilisées en routine pour la vérification de la stabilité au glissement, qui postulent des cercles de glissement; ces méthodes sont approximatives surtout concernant les argiles et les sollicitations transitoires.

La stabilité d'ensemble et la résistance au glissement d'un barrage en remblai reposent avant tout sur une bonne compréhension du contexte géologique et géotechnique, sur une conception adéquate (contrôle des écoulements, zonage des remblais avec des matériaux granulaires en recharge, purge ou traitement des matériaux inadéquats en fondation) et un contrôle soigné de la mise en œuvre (teneur en eau, compactage, pressions interstitielles).

\section{4itis}

\section{Calcul standard : évaluation de la stabilité par calcul à l'équilibre limite}

Le cas le plus fréquent est décrit ci-dessous (Fig. 4.1) :

1) un modèle de calcul à l'équilibre limite est bâti, qui postule une surface de glissement potentielle, traversant le remblai et, le cas échéant, la fondation;

2) on calcule les contraintes effectives le long de cette surface ;

3) on compare la contrainte de cisaillement, calculée le long de la surface étudiée, à la résistance au cisaillement des matériaux par différents modèles d'état limite (cf. 4.7.2.1) ; cette résistance est généralement exprimée par un modèle de Mohr-Coulomb, dans lequel on utilise pour $c^{\prime}$ et $\varphi^{\prime}$ les résistances de pic si, et seulement si, les preuves de compactage existent et que la déformabilité de la fondation est moindre que celle du remblai (sinon prendre les résistances résiduelles post-rupture) ;

4) la sécurité du calcul réside dans les trois points cidessous :

- $c^{\prime}$ et $\varphi^{\prime}$ sont choisis avec prudence (valeurs « caractéristiques 》), cf. $\$ 2.4 .6$ ) ;

- les valeurs de calculs de $c^{\prime}$ et $\varphi^{\prime}$ sont les valeurs caractéristiques, divisées par des coefficients partiels ;

- on applique ensuite un coefficient de modèle.

La condition d'état limite s'écrit sous la forme d'une inéquation dans laquelle sont comparés, d'une part, un rapport des forces résistantes (ou de leur moment) sur les forces motrices (ou de leur moment), d'autre part, le coefficient de modèle. L'expression mathématique de la condition d'état limite dépend du modèle d'état limite adopté ; elle fait intervenir les valeurs caractéristiques des propriétés de résistance pondérées par leur coefficient partiel et les valeurs représentatives des actions correspondant à la situation de projet examinée. A titre d'exemple, avec la méthode de Fellenius et pour un calcul en contraintes effectives, la condition d'état limite s'écrit :

$$
\frac{\sum_{i}\left[\frac{c^{\prime}}{\gamma_{m c^{\prime}}} \cdot \frac{b}{\cos \alpha_{i}}+\left(\frac{W_{i}}{\gamma_{m w}} \cos \alpha_{i}-u_{i} \frac{b}{\cos \alpha_{i}}\right) \frac{\tan \varphi^{\prime}}{\gamma_{m \tan \varphi^{\prime}}}\right]}{\sum_{i} W_{i} \sin \alpha_{i}}>\gamma_{\mathrm{d}}
$$


Situation normale d'exploitation de retenue pleine, à long terme

Situation normale d'exploitation de retenue basse, à long terme

Situation transitoire de fin de construction, et remplissage partiel

Situation rare de crue (barrages écrêteurs de crues)

Situation exceptionnelle de crue

Situation transitoire de vidange rapide

Situation rare d'altération du contrôle des écoulements

Situation rare de séisme SBE

Situation accidentelle de défaillance du contrôle des écoulements

Situation extrême de crue

Situation accidentelle de séisme SES
Établissement du régime permanent de piézométrie dans le corps du barrage à retenue pleine. La piézométrie est imposée par la retenue, et éventuellement la nappe et les précipitations

Abaissement normal (anmuel par exemple) du plan d'eau après que le régime permanent a été établi. Régime transitoire car les pressions interstitielles n'ont pas nécessairement eu le temps de se dissiper

Pour les remblais argileux ou sur sol compressible, cette situation peut correspondre à des maxima de pressions interstitielles, qui se seraient développées pendant la construction et ne se seraient pas dissipées

Établissement du régime permanent de piézométrie dans le corps du barrage ou calcul hydraulique en régime transitoire selon la durée des crues et la perméabilité des matériaux du remblai

Élévation du plan d'eau après que le régime permanent a été établi. Régime transitoire car les pressions interstitielles n'ont pas nécessairement eu le temps de s'établir

Abaissement rapide du plan d'eau après que le régime permanent a été établi. Attention aux premières vidanges des remblais très argileux

Les risques d'altération ou de rupture des dispositifs d'étanchéité ou de drainage sont évalués (dégradation d'un rideau, voile ou masque d'étanchéité, colmatage d'un drain...). On cherche ici le scénario possible d'altération, tenant compte des dispositions d'auscultation et d'entretien adoptées

Occurrence du séisme SBE alors que la retenue est pleine. Effet des efforts d'inertie, et surtout, dans certains cas, augmentation des pressions interstitielles voire liquéfaction

Les risques d'altération ou de rupture des dispositifs d'étanchéité ou de drainage sont évalués (dégradation d'un rideau, voile ou masque d'étanchéité, colmatage d'un drain...). On cherche ici le scénario accidentel de rupture, tenant compte des dispositions d'auscultation et d'entretien adoptées

Il peut notamment s'agir d'une rupture ou défaillance suite à des événements accidentels (séisme, malveillance...)

Atteinte de la cote de danger, qui conduit par ailleurs à un ELU d'érosion (interne ou externe)

Occurrence du séisme SMP alors que la retenue est pleine. Effet des efforts d'inertie, et surtout, dans certains cas, augmentation des pressions interstitielles voire liquéfaction
Glissement du talus aval

Soulèvement hydraulique du pied aval

Tassements excessifs

Glissement du talus amont

Ex. : Cercey, Liez

Glissement du talus amont ou du talus aval Défaut de portance de la fondation

Ex. : Mirgenbach, Mondely

Glissement du talus aval

Soulèvement hydraulique du pied aval

Glissement du talus aval

Soulèvement hydraulique du pied aval

Glissement du talus amont

Ex. : Mondely, Pessoulens

Glissement du talus amont ou du talus aval Soulèvement hydraulique du pied aval

Déformations excessives

Glissement du talus amont ou du talus aval Soulèvement hydraulique du pied ava

Glissement du talus aval

Glissement des talus (surtout aval)

Ex. : Lower San Fernando 
avec $b$ largeur des tranches, $W_{i}$ poids de la tranche $i, \alpha_{i}$ angle de la normale à la base de la tranche i avec la verticale, $W_{\mathrm{i}}$ pression interstitielle à la base de la tranche.

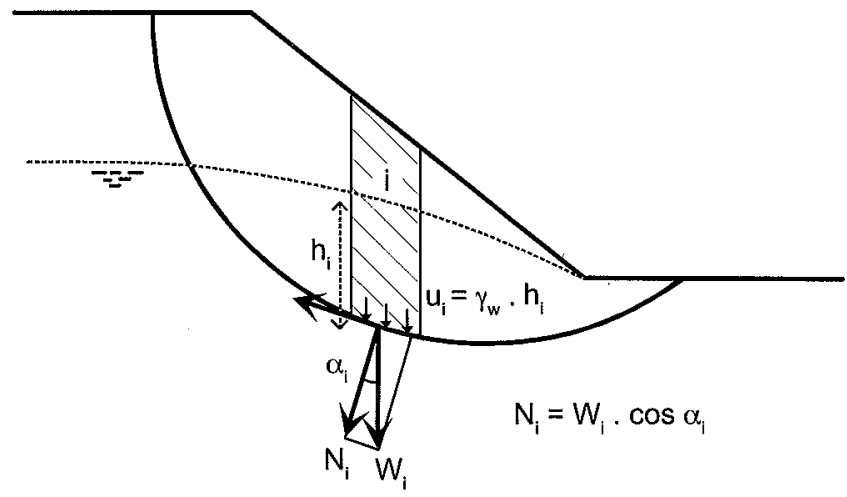

fic 4.1. Principe de calcul de la stabilité dans le cas d'une surface de rupture circulaire.

Les coefficients partiels et de modèle sont les suivants :

\section{3)}

\section{Modulations des règles du calcul standard}

Les règles ci-dessus sont modulées dans certains cas particuliers.

- Il est parfois utile ou nécessaire de procéder à une modélisation plus complète, par exemple par la méthode aux éléments finis (cf. § 4.7), en remplacement ou en complément du modèle de calcul à l'équilibre limite. Les calculs aux éléments finis permettent d'avoir une vision des déformations et des coefficients de sécurité locaux.

Les critères de stabilité peuvent rester de même nature que pour les calculs à l'équilibre limite : les calculs peuvent être effectués avec les valeurs caractéristiques du critère de plasticité et des modules de rigidité, minorées des mêmes coefficients partiels appliqués à $c^{\prime}$ et tan $\phi^{\prime}$ et présentés au tableau 4.II.

Pour les calculs complexes, il est parfois impossible de définir un coefficient de sécurité sur la loi de comportement; le jugement sur les marges de dimensionnement provient alors de l'analyse au cas par cas des déformations calculées.
- Pour la situation de fin de construction, en plus du calcul en contraintes effectives, il est possible de mener un calcul en contraintes totales en complément.

- Pour les barrages comportant des matériaux argileux plastiques, des précautions particulières doivent être prises (cf. § 4.7.4).

\section{5: \\ État limite de soulèvement hydraulique au pied aval}

L'état limite de soulèvement hydraulique au pied aval est à vérifier lorsque, en aval du barrage ou de la digue, la stratification géologique comporte une couche de sol peu perméable surmontant une ou des couches de sol plus perméables. Cela peut conduire à ce que s'établissent, sous cette couche de sol, des pressions d'eau pouvant la déstabiliser.

Le critère de stabilité s'exprime en équilibre : comparaison du poids de la couche de sol peu perméable et des pressions d'eau sous cette couche.

Cette situation est contrôlée par un drainage adéquat, qui consiste par exemple à percer la couche imperméable en pied aval (puits de décompression). C'est la solution pour les barrages.

Lorsque cela n'est pas possible, les conditions d'équilibre sont établies en considérant:

- la pression d'eau agissant sous la couche de sol, $u$;

- la contrainte totale apportée par le poids de la couche de sol, $\sigma_{v}$;

et en négligeant la cohésion.

La condition d'état limite s'écrit : $\gamma_{u} u>\gamma_{m} \sigma_{v}$

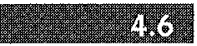

\section{État limite ultime de défaut de portance - État limite de service de tassement}

\section{4.}

\section{Introduction}

Les états limites de déformation pour un ouvrage hydraulique en remblai correspondent essentiellement à des critères de fonctionnalité (l'état limite de service de tassement). Il est essentiel de préserver l'intégrité

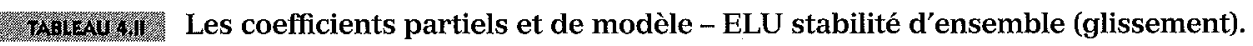

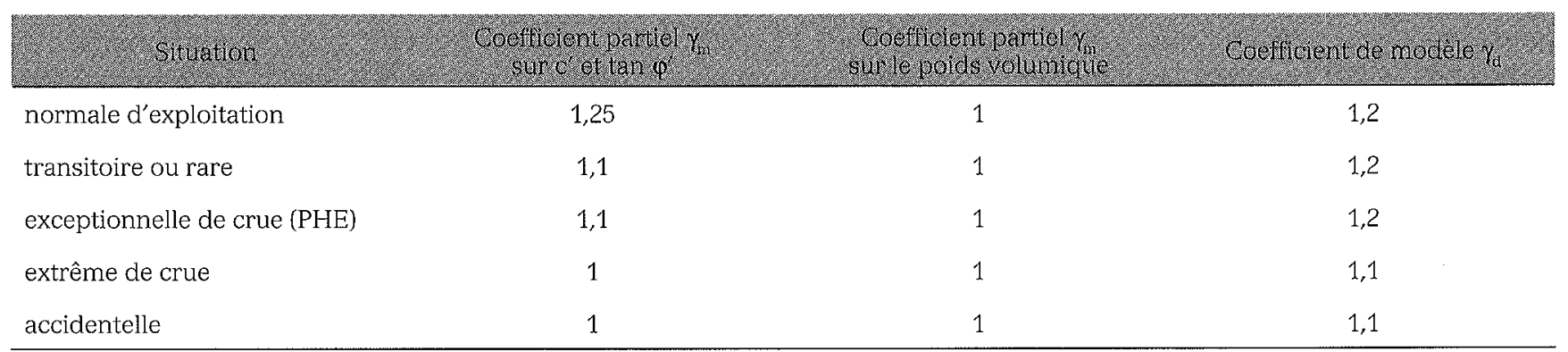


T.

\begin{tabular}{|c|c|c|}
\hline (25) & 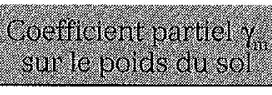 & 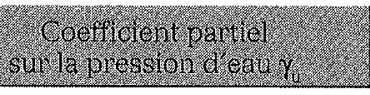 \\
\hline normale d'exploitation & 0,90 & 1,2 \\
\hline transitoire ou rare & 0,90 & 1,2 \\
\hline exceptionnelle de crue (PHE) & 0,90 & 1,2 \\
\hline extrême de crue & 1 & 1,1 \\
\hline accidentelle & 1 & 1,1 \\
\hline
\end{tabular}

des organes vitaux des ouvrages hydrauliques en remblai que sont l'étanchéité (noyau argileux, masque amont, écran interne), le système de drainage (drain vertical ou incliné, tapis drainant) et les conduites et galeries traversantes.

L'objectif est donc de se prémunir de déformations excessives du remblai ou de sa fondation pouvant entraîner :

- une diminution de la revanche et donc un risque de rupture par érosion externe ;

- une modification du réseau d'écoulement interne (par exemple par cisaillement du système de drainage suite à des tassements différentiels) et donc un risque de rupture par glissement;

- une ouverture de fissures dans l'organe d'étanchéité (par exemple par fracturation hydraulique pour un barrage à noyau ou par rupture de l'écran d'étanchéité d'un barrage à masque) et donc un risque de rupture par érosion interne ;

- une fissuration ou une rupture des conduites ou galeries passant dans le remblai ou la fondation.

Des déformations excessives sont également susceptibles d'entraîner un mécanisme de rupture de l'ouvrage en remblai, correspondent à des critères de résistance de la fondation, appelés état limite ultime de défaut de portance.

On distingue donc l'état limite de défaut de portante de la fondation (ELU) et l'état limite de tassement du remblai (ELS).

\section{5}

\section{État limite ultime de défaut de portance de la fondation}

La notion de portance (ou de capacité portante) de la fondation fait référence à un critère de rupture en cisaillement de la fondation pour des ruptures par poinçonnement. Ce sont des ruptures du sol de fondation caractérisées par le fait que le remblai s'affaisse en subissant des tractions. La rupture du sol de fondation est générale puisqu'elle intéresse la totalité de la largeur d'assise du remblai. Le schéma de rupture du sol de fondation est analogue à celui qui se produit sous une fondation superficielle et peut donc être étudié comme tel.

Le cas le plus défavorable est de manière générale la fin de construction (court terme). La vérification de la stabilité au poinçonnement devra donc se faire en supposant le remblai construit instantanément, sans dissipation de pressions interstitielles dans le sol de fondation. Seuls les ouvrages neufs ou les projets de surélévation d'ouvrages anciens sont concernés.

La contrainte verticale q sous un remblai de hauteur $\mathrm{H}$ et de poids volumique $\gamma$ peut être approximée par :

$$
\mathrm{q}=\gamma \mathrm{H}
$$

La contrainte maximale admissible sur la fondation $q_{\max }$ en fonction de sa cohésion non drainée $c_{u}$ peut être exprimée par :

$$
q_{\max }=(\pi+2) c_{u}
$$

Cette formulation est valable pour une fondation superficielle de dimension réduite sur une couche épaisse de sol compressible. Lorsque le remblai est large vis-à-vis de l'épaisseur du sol compressible, le coefficient $(\pi+2)$ n'est plus acceptable. Mandel et Salençon (1969) ont proposé une solution dans laquelle la pression limite sur un sol de cohésion $\mathrm{C}_{\mathrm{u}}$ s'exprime par :

$$
\mathrm{q}_{\max }=\mathrm{C}_{\mathrm{u}} \mathrm{N}_{\mathrm{c}}
$$

avec $N_{c}$ coefficient fonction de $B / D$ où $B$ est la largeur moyenne du remblai à mi-hauteur et $\mathrm{D}$ l'épaisseur de fondation compressible. $\mathrm{N}_{\mathrm{c}}$ se détermine à partir d'abaques mais peut être approximé par $4+0,5 \mathrm{~B} / \mathrm{D}$.

\section{La condition d'état limite s'écrit :}

$$
\mathrm{q}_{\max } / \gamma_{\mathrm{m}}>\gamma_{\mathrm{d}} \mathrm{q}
$$

avec $\gamma_{\mathrm{m}}$ coefficient partiel sur la cohésion non drainée et $\gamma_{d}$ coefficient de modèle.

Cet état limite n'est à considérer que pour la situation transitoire de fin de construction. On adopte une valeur de 1,4 pour $\gamma_{\mathrm{m}}$ (valeur proposée dans l'Eurocode 7) et de 1,2 pour $\gamma_{\mathrm{d}}$ (valeur adoptée précédemment pour les autres ELU en situation transitoire ou rare).

Cet état limite peut aussi être analysé comme un état limite de glissement en considérant des cercles profonds passant par la fondation. Il faut cependant se méfier des matériaux de fondation compressibles qui nécessitent un taux de déformation important pour que la résistance maximale au cisaillement soit mobilisée. Il peut être utile alors de tenir compte des caractéristiques de résistance au cisaillement de la fondation pour un taux de déformation donné, pouvant correspondre au taux de déformation mobilisant la résistance au pic des matériaux du remblai. D’une manière générale, il faut s'interroger sur les caractéristiques de résistance à adopter dès lors que le taux de déformation nécessaire pour mobiliser la résistance maximale au cisaillement dépasse $5 \%$. L'utilisation des valeurs résiduelles de résistance au cisaillement est conseillée. 
Un calcul par éléments finis doit être effectué dès lors que le remblai dépasse la dizaine de mètres de hauteur ou si, en utilisant la formulation précédente, la fondation présente un risque d'instabilité. Le phasage de construction doit alors être pris en compte, tout comme le phénomène de consolidation de la fondation, via des lois de comportement adaptées. Une analyse préliminaire peut être réalisée en prenant en compte les caractéristiques non drainées des matériaux (calcul en contraintes totales).

\section{6.}

\section{État limite de service de tassement (ELS)}

L'état limite de tassement correspond à un critère de fonctionnalité. L'objectif est que l'intégrité des organes d'étanchéité et de drainage soit maintenue. Le risque est que des déformations excessives conduisent à des fissures voire des ruptures sur des galeries ou conduites, ou amènent l'ouvrage dans un état de danger potentiel vis-à-vis des ruptures par glissement, érosion interne et érosion externe.

\section{On distingue :}

- les situations normales d'exploitation et les situations transitoires ou rares. Les déformations du remblai et de sa fondation étant le plus souvent irréversibles, l'efficacité des systèmes d'étanchéité et de drainage ne doit en aucun cas être impactée par les déformations engendrées par ces sollicitations;

- les situations accidentelles. Dans le cas d'ouvrages dont les systèmes d'étanchéité et/ou de drainage peuvent être réparés (masque étanche amont sur barrage en enrochements par exemple), il peut être admis que les situations accidentelles conduisent à une perte partielle ou totale d'efficacité de ces systèmes (fissures du masque). On doit alors vérifier que la stabilité de l'ouvrage est assurée avec la sécurité suffisante. Dans le cas d'ouvrages dont les systèmes d'étanchéité et/ou de drainage ne peuvent être réparés sans travaux majeurs (barrage à noyau argileux par exemple), il est essentiel de garantir l'efficacité de ces systèmes (maintien de l'intégrité du noyau et des drains). On peut considérer que l'ouvrage en condition post-situation accidentelle doit dans tous les cas pouvoir revenir rapidement à une situation durable d'exploitation, moyennant travaux de remise à niveau.

Il n'existe pas de critères sur les déformations admissibles des remblais. La valeur de la déformation admissible pour un ouvrage hydraulique en remblai dépend directement du type d'ouvrage et notamment de la déformabilité du système d'étanchéité et de drainage de cet ouvrage. Par exemple, un masque en béton ne pourra supporter des déformations supérieures à quelques \% alors qu'un noyau en argile peut généralement supporter des déformations de quelques \%.

Pour les propriétés des matériaux de remblai, on n'utilise pas de coefficients partiels mais les propriétés sont estimées de manière prudente au moyen des valeurs caractéristiques. On peut aussi mener une étude paramétrique en appliquant des réductions forfaitaires aux paramètres estimés (approche $c / \varphi$ réduction).
Dans la pratique, on peut prendre en compte l'incertitude de la façon suivante : on applique un coefficient majorateur sur les déformations ou les déplacements calculés et on examine s'ils sont admissibles pour les organes concernés (conduite, étanchéité mince, drain...).

Suivant la problématique identifiée, l'estimation des déformations peut se faire :

- par calcul analytique unidimensionnel (problématique liée à la diminution de la revanche de l'ouvrage), une valeur de tassement maximal égale à $1 \%$ de la hauteur de l'ouvrage est souvent considérée pour des études préliminaires. Lors de la construction d'un ouvrage neuf, la valeur de tassement maximal est adoptée pour le dimensionnement de la contre-flèche;

- par calcul EF bidimensionnel dans une section longitudinale RD-RG (problématique liée à une fissuration transversale due à des zones de faiblesse de la fondation ou à une fissuration horizontale du noyau étanche due à un report de contraintes vers les rives dans une vallée étroite) ;

- par calcul EF bidimensionnel dans une section transversale amont-aval (problématique liée à une fissuration horizontale du noyau étanche due à un report de contraintes vers les transitions amont et/ou aval plus raides que le noyau, ou à une fissuration longitudinale due à des tassements différentiels noyau/recharge) ;

- par calcul EF tridimensionnel dans le cas où les différents phénomènes cités se cumulent, et dans le cas où la problématique ne peut se simplifier en une étude bidimensionnelle (hétérogénéité complexe de fondation, géométrie de vallée particulière - par exemple courbe ou très encaissée -, ouvrage en contact avec des structures béton, etc.).

\section{1.}

\section{Calcul analytique unidimensionnel}

Ce calcul revient à estimer le tassement de consolidation d'une colonne de remblai et de sa fondation sous le poids propre de la colonne. La vitesse de montée du remblai doit être prise en compte.

\section{62}

\section{Calcul EF bidimensionnel dans une section longitudinale RD-RG}

La section modélisée dépend du type d'ouvrage et doit être adaptée suivant les problématiques identifiées. Dans le cas d'un barrage à noyau central argileux, la section étudiée est la section dans l'axe du barrage.

Seule la situation de fin de construction peut être modélisée (chargement dans le plan vertical). Pour la problématique liée à la fissuration horizontale, on compare les contraintes verticales obtenues par le calcul aux pressions interstitielles attendues en fin de remplissage. De façon simpliste, si en un point du noyau les contraintes totales verticales en fin de construction sont inférieures ou égales aux pressions interstitielles une fois le réservoir rempli, il y a risque de fracturation hydraulique.

La distribution des contraintes ayant une importance primordiale dans cette problématique, la modélisation devra a minima tenir compte du phasage de construction. L'emploi d'un modèle de comportement élastoplastique est souhaitable. 
La mise en place d'un matériau plus plastique au contact avec la fondation (ou une structure béton adjacente) pourra être modélisée soit par un matériau aux propriétés mécaniques différentes, soit par une interface de glissement.

\section{6)}

\section{Calcul EF bidimensionnel dans une section transversale amont-aval}

Les phases de construction par couches et de remplissage du réservoir doivent être modélisées.

La distribution des contraintes ayant une importance primordiale, l'emploi d'un modèle de comportement élastoplastique et la prise en compte du couplage hydromécanique (pour les barrages à noyau argileux seulement) sont particulièrement souhaitables.

\section{5}

\section{Calcul EF tridimensionnel}

Le calcul tridimensionnel est conseillé pour évaluer le comportement de grands barrages zonés établis dans des vallées étroites ou dissymétriques. Il permet d'analyser le risque de claquage hydraulique par report de charges du noyau sur les filtres et les recharges.

\section{1...}

\section{La fissuration des remblais}

Les fissures se développent dans des zones en traction au sein du remblai. Ces tractions sont généralement causées par des tassements différentiels intervenant pendant et après la construction, durant les phases de remplissage/vidange du réservoir et durant les séismes. On distingue trois types de fissures : transversale (verticale et dans une direction amont-aval), horizontale, longitudinale (verticale et dans une direction rive à rive) :

a) transversales : les fissures transversales dans un noyau imperméable sont très préjudiciables à la sécurité de l'ouvrage puisqu'elles créent des chemins d'eau à travers le remblai (risque de rupture par érosion interne). Les fissures transversales peuvent être causées par des contraintes de traction résultant de tassements différentiels du remblai et/ou de sa fondation. Les tassements différentiels peuvent apparaître au contact avec des rives très raides, au contact avec des structures en béton adjacentes, là où le compactage du remblai est difficile, ou au-dessus de zones compressibles en fondation (anciens méandres de rivières par exemple). Ils peuvent aussi apparaître dans la recharge amont à la mise en eau, par lubrification des contacts entre enrochements ;

b) horizontales : les fissures horizontales dans un noyau imperméable apparaissent quand le matériau du noyau est beaucoup plus compressible que les matériaux adjacents. Le noyau a alors tendance à s'accrocher sur les matériaux plus raides, d'où un transfert de poids vers ces matériaux et par conséquent une réduction de la contrainte effective verticale au sein du noyau. Lors du remplissage du réservoir, la pression s'installe et peut annuler la contrainte effective verticale au sein du noyau, ouvrant ainsi une fissure. Ce phénomène est appelé fracturation hydraulique. Ces fissures ne sont pas visibles depuis l'extérieur ; c) longitudinales : les fissures longitudinales peuvent résulter de tassements excessifs de la recharge amont lors du premier remplissage d'un réservoir (phénomène d'effondrement des enrochements sous mouillage) ou lors d'une vidange rapide. Elles peuvent être causées également par des tassements différentiels entre matériaux adjacents ou sous l'effet d'un séisme. Enfin, comme indiqué au $\$ 4.6 .2$, des fissures longitudinales peuvent se développer en cas de défaut de portance de la fondation. Ces fissures n'ouvrent pas de chemins d'eau de l'amont vers l'aval et ne créent donc pas le risque d'érosion interne. Par contre, elles peuvent diminuer la stabilité au glissement des remblais, particulièrement si les fissures se remplissent d'eau.

\section{1)}

\section{Contre-flèche}

Une contre-flèche est généralement prévue le long de la crête d'un barrage en remblai pour garantir que la revanche ne sera pas diminuée par les tassements de l'ouvrage et de sa fondation. La valeur de la contreflèche est de fait arbitraire. Elle est basée sur l'estimation des tassements de l'ouvrage et de sa fondation avec pour objectif d'être suffisante pour qu'une contreflèche résiduelle soit conservée après tassements et consolidation. Cette contre-flèche résiduelle améliore également l'apparence visuelle de la crête.

Les matériaux imperméables mis en place à des densités proches de l'optimum Proctor sont généralement sujets à des tassements de consolidation conséquents lorsqu'ils sont chargés par les couches sus-jacentes. Pour les petits barrages, en supposant une vitesse raisonnable de montée du remblai, la consolidation primaire de ces matériaux est souvent pratiquement terminée avant la fin de construction de l'ouvrage. Par conséquent, ce sont les tassements de fondation qui sont prépondérants dans l'estimation de la contre-flèche.

Pour des barrages sur des fondations peu compressibles, une contre-flèche de l'ordre de $1 \%$ de la hauteur du remblai est communément adoptée. Une fois déterminée la contre-flèche dans la section principale de l'ouvrage (section la plus haute sur fondation), on adopte une variation linéaire de celle-ci avec la hauteur du remblai.

\section{7}

\section{Principes de mise en œuvre des calculs}

\section{This}

\section{Les modèles rhéologiques}

Des lois simplifiées (pour une approche sommaire) ou plus évoluées (utilisables notamment dans les codes de calculs aux éléments finis), reliant contraintes et déformations dans les sols, permettent d'obtenir une bonne image du comportement réel des sols. Les modèles les plus couramment utilisés sont:

- les modèles élastiques linéaires parfaitement plastiques (avec critère de rupture de Mohr-Coulomb, de Von Mises...). Ces modèles sont pratiques car ils ne nécessitent le calage que de quelques paramètres, mais ils peuvent sous-estimer les déplacements ; 
- les modèles élastiques non linéaires, dont le modèle hyperbolique (Duncan-Chang). Les plus pratiques pour l'analyse statique des remblais en terre, ils sont déconseillés pour étudier les remblais en enrochements à noyau d'argile ou à masque amont (matériaux où la dilatance et le radoucissement sont suspectés). Ils trouvent également leur limite pour des simulations sous chargement non monotone;

- les modèles élastoplastiques avec écrouissage (tel le modèle de Cam-Clay). Moyennant l'utilisation de paramètres bien ajustés (représentatifs notamment du chemin de contrainte imposé aux matériaux et déterminés par l'intermédiaire d'essais triaxiaux), ces modèles permettent la meilleure représentation du comportement des barrages en remblai ;

- le modèle rigide-plastique : uniquement valable pour les roches saines avec un module d'élasticité très élevé. Le matériau est rigide (aucune déformation) dans le domaine des contraintes inférieures à une contrainte limite $\sigma_{1}$ et a un comportement plastique parfait lorsque l'on applique $\sigma_{r^{r}} c^{\prime}$ est-à-dire que le sol se déforme alors que la contrainte reste égale à $\sigma_{l}$.

\section{the}

\section{Calcul d'équilibre limite et calcul à la rupture}

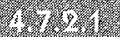

\section{Méthodes et outils de calculs}

Les méthodes de calcul d'équilibre limite consistent à découper le volume glissant en tranches verticales. Les méthodes diffèrent notamment sur les hypothèses de transmission des efforts entre les tranches. Les calculs sont en général bidimensionnels.

Quatre méthodes sont utilisées en pratique : Bishop simplifiée (rupture circulaire uniquement), Morgenstern-Price (rupture non nécessairement circulaire), Janbu simplifiée et méthode des perturbations. Il faut éviter la méthode de Fellenius dont les hypothèses sont trop simplificatrices.

On peut également mentionner la méthode cinématique de Salençon qui calcule des majorants des forces d'équilibre.

Dans les cas où la fondation est soumise à des pressions artésiennes ou à une rigidité plus faible que le remblai, il convient de comparer ces résultats avec une approche en déformation. En effet, la présence de zones en traction rend non valides les hypothèses des méthodes des tranches.

Les méthodes de calcul à l'équilibre limite sont toutes basées sur les hypothèses suivantes:

- la forme et la position de la ligne de rupture sont choisies à l'avance ;

- le sol a un comportement rigide plastique le long de cette ligne ;

- la distribution des contraintes est connue ;

- le rapport entre la résistance au cisaillement mobilisable le long de la ligne de rupture et la résistance au cisaillement nécessaire à maintenir la masse potentiellement glissante en équilibre (dite aussi résistance au cisaillement mobilisée) divisé par le coefficient de modèle donne accès au facteur de sécurité ;

- le facteur de sécurité est uniforme le long de la surface de rupture (i.e. le matériau traversé par la surface de rupture est unique et homogène).
Dès que l'une ou l'autre de ces hypothèses est jugée inacceptable en rapport aux conditions réelles, il conviendra de comparer les résultats avec une analyse en contrainte-déformation via la méthode des éléments finis. On citera notamment le cas classique où une couche molle est présente en fondation ou dans le remblai. De nombreux outils de calculs sont commercialisés. Il faut privilégier les outils qui permettent de bien modéliser l'action des pressions interstitielles : dans de nombreux cas, il ne suffit pas de rentrer la ligne de saturation. Il faut en effet pouvoir :

- récupérer ou spécifier un réseau d'écoulement;

- récupérer ou spécifier un champ de pressions interstitielles.

Il convient d'être particulièrement prudent lorsque la ligne de saturation se rapproche du talus aval, les résultats pouvant alors fortement varier selon la méthode employée.

Lors de l'étude, il faut tester de nombreux cercles de rupture différents, pour confirmer l'unicité du cercle dont le facteur de sécurité est minimal. Outre des cercles de rupture profonds, on recherche également des cercles représentatifs de la stabilité de peau, pour autant que cela corresponde à une réalité physique.

\section{1.}

\section{Mise en cuure des calculs}

Les calculs nécessitent de préciser la géométrie des différentes couches de sol et zones de remblais, leurs caractéristiques mécaniques $\left(c^{\prime}, \tan \varphi^{\prime}\right)$, les éventuelles actions extérieures (surcharges, séisme...) et le champ de pressions interstitielles. Le résultat du calcul est un facteur de sécurité qu'il convient de comparer au coefficient de modèle requis selon le type de situation (tableau 4.II).

Lorsque le talus aval est protégé (enrochements, enherbement sur couche drainante), cette protection agit sur la stabilité, eu égard aux cercles de peau. On peut donner à cette protection (ou au remblai de la recharge si cette protection n'est pas modélisée) une petite cohésion (quelques $\mathrm{kPa}$ ), qui empêche que le calcul ne soit perturbé par des cercles de peau sans réalité physique.

Le facteur de sécurité ne varie pas linéairement avec les valeurs de $c^{\prime}$ ou $\tan \varphi^{\prime}$ ou avec les valeurs de pression interstitielle. Pour apprécier les marges de dimensionnement, il est recommandé de procéder à des études de sensibilité concernant les principaux paramètres de calcul.

\section{(5)}

\section{Limites du calcul à l'équilibre limite}

Le calcul à l'équilibre limite ne peut plus être utilisé lorsque le comportement du barrage est piloté par les déformations. C'est notamment le cas :

- de la stabilité d'ensemble d'un barrage fondé sur des matériaux compressibles (cf. $\S 4.6$ ), davantage encore lorsque le barrage est constitué de remblais d'époques de construction différentes ;

- des matériaux à forte perte de résistance post-pic (cf. $\$ 4.7 .4$ ) ;

- des situations de rupture progressive. 
Le calcul à l'équilibre limite 2D ne convient plus non plus lorsque les effets 3D prédominent (vallée étroite) ou lorsque les surfaces de glissement sont difficiles à évaluer a priori (c'est le cas des géométries complexes).

\section{the}

\section{Calcul aux éléments finis}

\subsection{Th}

\section{Méthodes et outils de calcul}

La méthode des éléments finis consiste à calculer les contraintes et déformations à l'aide d'une modélisation en éléments finis. Cette modélisation est une opération beaucoup plus complexe que le calcul à l'équilibre limite ; il est plus difficile d'assurer le contrôle des données d'entrée et de s'assurer de la pertinence des résultats. Or les calculs convergent presque toujours vers une solution, parfois fausse, mais en général donnant l'illusion de la validité. Aussi, un calcul aux éléments finis ne devrait jamais être réalisé sans une validation, par exemple par des calculs simplifiés d'ordre de grandeur.

Les calculs doivent être menés avec des logiciels adaptés à la géotechnique, et notamment qui permettent au moins de :

- modéliser les lois de comportement élastique parfaitement plastique avec critère de Mohr-Coulomb ;

- modéliser le phasage de construction, qui détermine les états de contraintes dans les matériaux;

- prendre en compte les effets de l'eau interstitielle (pressions, gradients);

- si nécessaire (interface avec les ouvrages rigides), modéliser les non-linéarités de contact à ces interfaces ;

- si nécessaire (présence de matériaux fins), modéliser la consolidation.

\section{7.}

\section{Mise en cuure}

Le modèle doit représenter le barrage, sa fondation et la retenue.

La modélisation impose de reproduire, dans le calcul, le phasage de construction et de mise en eau.

Les résultats sont obtenus en termes de contraintes et de déformations. Cela ne donne pas directement accès au facteur de sécurité. Pour obtenir le facteur de sécurité de l'ouvrage, la méthode habituelle consiste à réduire $c^{\prime}$ et $\tan \varphi^{\prime}$ (ou les paramètres de la loi de comportement) jusqu'à ce que le calcul ne converge plus. Ce facteur de sécurité peut être comparé au produit (coefficient partiel x coefficient de modèle) pour se rapprocher du calcul standard décrit au § 4.4.2.

Dans le cas de la modélisation de barrages en service, on s'attache à qualifier le modèle par comparaison avec les mesures d'auscultation. On pourra ensuite actualiser le ( modèle de l'ouvrage ) lors des études de dangers.

\section{1.}

\section{Difficultés de la modélisation des argiles}

Les argiles posent trois difficultés de modélisation : - peu perméables, elles génèrent des surpressions de construction ;

- elles résistent au cisaillement essentiellement par leur cohésion ; or cette cohésion est difficile à mesurer, et s'altère dans le temps (gonflement par la saturation, dessiccation...);

- leur résistance résiduelle (après cisaillement) est parfois très inférieure à leur résistance de pic.

Ces difficultés font que l'on évite, dans la mesure du possible, les barrages homogènes en argile : il est nettement préférable d'encadrer l'argile par des recharges granulaires. Lorsque ce n'est pas possible, il est au moins recommandé de protéger les argiles de la dessiccation (coupure capillaire).

Les difficultés sont d'autant plus importantes que les argiles sont plastiques, en particulier lorsque l'indice de plasticité $\mathrm{I}_{\mathrm{p}}$ dépasse 25.

Pour la modélisation des argiles, on peut retenir les règles suivantes :

- pour une argile non consolidée, la cohésion à long terme $\mathrm{c}^{\prime}$ est nulle; le compactage de l'argile apporte une contrainte de préconsolidation qui est de l'ordre de 100 à $250 \mathrm{kPa}$, suivant la teneur en eau initiale ;

- si l'argile est exposée aux sollicitations climatiques (notamment cycles de saturation/désaturation), elle évolue et peut progressivement perdre sa cohésion ;

- en général, les argiles sont modélisées avec leurs résistances de pic et un critère bilinéaire (surconsolidé $\sigma^{\prime}<\sigma_{c}^{\prime}$ et normalement consolidé $\sigma^{\prime}>\sigma_{c}^{\prime}$ ). Cependant :

- lorsque l'écart entre résistance de pic et résistance résiduelle est important, le risque de rupture progressive doit être évalué ; cela impose soit de vérifier l'ouvrage avec les résistances résiduelles des argiles, soit de procéder à un calcul aux éléments finis qui permette d'évaluer si le risque existe (le calcul à la rupture postule une répartition homogène du cisaillement le long de la ligne de glissement, alors qu'en réalité il peut se produire des concentrations de contraintes),

- lorsque la fondation du barrage comporte des argiles surconsolidées, il faut tenir compte de la possibilité de réactivation de cisaillements anciens (rupture du barrage de Carsington).

\section{5 \\ Justification de la sécurité des digues à charge permanente}

1.8.

\section{Généralités}

Pour les digues à charge permanente ou non, on appelle amont le côté rivière et aval le côté terres (ou coté val). Amont et aval s'entendent ici sur une coupe en travers de la digue et non le long du cours d'eau ou du canal.

Les dispositions de cette partie s'appliquent aux digues à charge permanente telles que les digues de 
canaux navigables ou d'aménagements hydro-électriques. Ces ouvrages sont considérés comme des barrages au sens de la nouvelle réglementation sur les ouvrages hydrauliques (décret du 11 décembre 2007). Ils s'en distinguent principalement par leur conception (utilisation de dispositifs de protection de berges particuliers) et leurs conditions aux limites (prépondérance des actions liées à leur exploitation telles que le batillage). Les états limites ultimes qu'il convient de considérer pour ces digues à charge permanente sont:

- l'état limite ultime de stabilité d'ensemble (glissement) ;

- l'état limite ultime de soulèvement hydraulique au pied aval ;

- l'état limite ultime de défaut de portance (poinçonnement de la fondation);

- l'état limite de service de tassement;

- l'état limite ultime d'affouillement en pied amont;

- les états limites de boulance, de résistance à l'érosion interne et de résistance à la surverse (traités ultérieurement) ;

- l'état limite de service de filtration (traité ultérieurement) ;

- l'état limite de liquéfaction sous sollicitation sismique (traité au sein d'un autre GT).

On traite également de l'état limite d'érosion de berge par les sollicitations dues au courant, au vent et à la navigation, mais on considère qu'il s'agit d'un ELS, les dégradations étant progressives et ne devant pas, sous réserve d'une surveillance et d'une maintenance adaptées, conduire à la rupture de l'ouvrage.

- Pour les états limites de stabilité d'ensemble, de soulèvement du sol et de défaut de portance de la fondation, seules les spécificités des digues en charge permanente sont traitées dans ce chapitre et il convient de se référer au chapitre 4 « Justification de la sécurité des barrages en remblai m pour les dispositions communes avec les barrages en remblai. Les états limites de boulance, d'érosion interne et de surverse seront traités dans une autre publication.

- Pour les situations de projet, il convient de se référer à la partie 1 de ces recommandations. Le cas échéant, les situations de projets doivent tenir compte du fait que l'ouvrage est édifié sur un linéaire délimité de part et d'autre par des ouvrages hydrauliques (équipés ou non d'écluses) permettant notamment de limiter l'extension des eaux.

- Pour les données géotechniques, il convient de se référer aux indications de la partie 2 de ces recommandations. Pour les digues existantes, il est souvent difficile d'avoir une vision claire de la constitution des ouvrages et de leurs fondations. Avant de procéder à des modélisations hydrauliques complexes (sur la base de caractéristiques rarement mesurées), la priorité doit être donnée à la connaissance réelle des matériaux (contrastes des perméabilités, anisotropie) et à l'instrumentation de l'ouvrage. Il convient de s'intéresser aux données quantitatives simples (granulométrie, indice de plasticité, densité) et aux essais in situ. Avant de procéder aux calculs aux états limites, il est recommandé de procéder à une analyse exhaustive des informations se rapportant à l'historique de l'ouvrage, son fonctionnement, sa constitution, et aux éventuels modes de rupture passés. L'effet sur la digue de la végétation (en particulier des arbres), des galeries creusées par les animaux fouisseurs et de points singuliers (conduites traversantes, raccordement à des ouvrages de génie civil, écluses...) est évalué de manière qualitative.

De nombreuses digues de canaux intègrent un écran de soutènement en palplanches métalliques. Lors de la justification de leur stabilité, les états limites à étudier sont :

- ceux de la digue du canal (cf. ce chapitre des recommandations);

- ceux de l'écran de soutènement. Nous précisons dans l'annexe 3 les états limites à vérifier pour un rideau de palplanches métalliques et pour plus de détails, il convient de se reporter à la norme NF EN 1993-5 (Eurocode 3, partie 5) ou à ROSA 2000 (fascicule « Rideaux de soutènement ) ou fascicule (c Exemples commentés de notes de calcul - Rideau de soutènement ancré s). Pour cette justification, il convient de tenir compte de l'influence des affouillements sur l'évolution du fond du lit (érosion des matériaux en pied de berges et diminution de la butée de pied).

Pour les canaux construits en remblai et jouant le rôle de protection contre les crues (cas des canaux latéraux aux rivières), il convient d'étudier distinctement:

- les états limites de la digue du canal en se référant à ce chapitre des recommandations ;

- les états limites du canal comme ceux d'une digue de protection contre les inondations (\$6).

\section{2}

\section{Les situations de projet}

Les situations de projet à analyser, ainsi que les états limites correspondants (hors érosion interne) sont présentés dans le tableau 5.I.

\subsubsection{3 \\ État limite de stabilité d'ensemble (glissement)}

La méthode de justification de la stabilité des digues à charge permanente vis-à-vis de l'état limite de glissement est identique à celle utilisée pour les barrages. Pour le calcul à long terme, lors d'une vidange contrôlée, on peut généralement considérer que la ligne de saturation est horizontale (niveau normal des eaux) dans la zone étanche de la digue (talus amont ou zone centrale) jusqu'au système de drainage aval. Cette approximation n'affecte pas sensiblement le résultat du calcul de stabilité, qui est également peu affecté par une variation du poids volumique des matériaux.

En alternative à la méthode des coefficients partiels, une étude de sensibilité adaptée à l'état limite de glissement (glissement généralisé) peut être effectuée (ROSA 2000).

Pour la vérification des digues existantes vis-à-vis de l'état limite de glissement, il est possible de s'appuyer sur le fait que la digue est toujours en place. Cette information peut être valorisée de deux manières :

- en comparant la section la plus défavorable aux autres sections, des facteurs de sécurité sont alors calculés pour les autres sections ; 


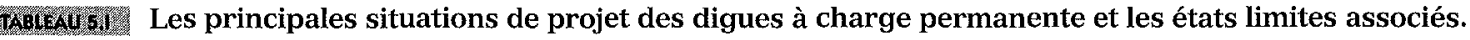

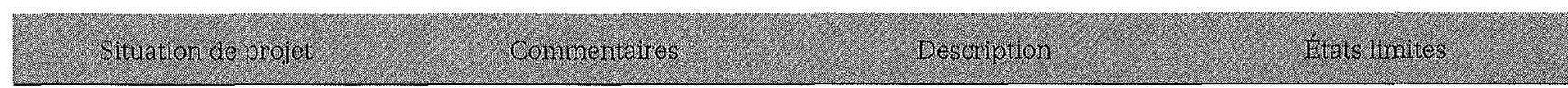

SITUATIONS NORMALES D'EXPLOITATION

Situation normale d'exploitation
Niveau normal d'exploitation (NNE) ou niveau normal de navigation (NNN) Cas particuliers d'ondes liées à la disjonction d'usine
Établissement du régime permanent de piézométrie dans le corps de la digue à niveau normal d'exploitation. La piézométrie est imposée par le niveau du canal et éventuellement la nappe et les précipitations

Glissement du talus aval Glissement du talus amont (cas particulier de disjonction d'usine)

Affouillement pied amont Soulèvement hydraulique en pied aval

\section{SITUATIONS TRANSITOIRES OU RARES}

Situation (transitoire) de fin de construction

Situation (transitoire) de vidange contrôlée

Situation accidentelle de séisme (SES)

Situation rare de séisme (SBE) Situation considérée uniquement pour les digues de classe A situées en zones sismiques 2 à 5

Pour ouvrages neufs ou
ouvrages existants après
vidange ou avec rechargement
(ou baisse prolongée de la
nappe)
Caractéristiques mécaniques
à court terme
Depuis le niveau d'exploitation
ou niveau normal
de navigation

Pour les remblais argileux ou sur sol compressible, cette situation peut correspondre à des pressions interstitielles, générées pendant la construction et qui ne se seraient pas dissipées

Vidange contrôlée du bief après que le régime permanent a été établi
Glissement du talus aval. Glissement du talus amont Défaut de portance de la fondation

Glissement du talus amont Affouillement pied amont (si augmentation des vitesses du courant)

SITUATIONS SISMIQUES (voir recommandations spécifiques)

\section{SITUATIONS LIÉES À LA DÉFAILLANCE D’UN COMPOSANT}

Situation (rare ou accidentelle) de défaillance du contrôle des écoulements

Situation (rare ou accidentelle) de vidange rapide

Situation (transitoire) de travaux en pied de digue
On cherche ici les scénarios de défaillance (rares ou accidentels selon leur probabilité d'occurrence), tenant compte des dispositions d'auscultation et d'entretien adoptées.

Il peut notamment s'agir d'une rupture ou défaillance suite à événements extérieurs (crues, séisme, malveillance...) ou liés au vieillissement

Depuis le niveau normal d'exploitation ou le niveau normal de navigation

Situation à éviter, voire à proscrire
Les risques d'altération ou de rupture des dispositifs d'étanchéité et de drainage sont évalués (dégradation d'un rideau, voile ou masque d'étanchéité, colmatage d'un drain...)

\section{Glissement des talus}

(surtout aval)

Liquéfaction

Glissement des talus

(surtout aval)

Déformation/tassement
Glissement du talus aval Soulèvement hydraulique en pied aval
Vidange rapide du bief suite à une rupture de digue ou fausse mancuvre d'un ouvrage hydraulique avec un niveau piézométrique maintenu dans la digue au niveau normal

Par exemple creusement d'une tranchée en pied de digue
Glissement du talus amont Affouillement pied amont (si augmentation des vitesses du courant)

Glissement du talus aval Défaut de portance de la fondation
- en calculant un gain sur le facteur de sécurité (plutôt que de viser les valeurs réglementaires) si un renforcement est envisagé. Selon le cas, on pourra rechercher un gain de $+20 \%$ à $+30 \%$.

Dans les cas où la protection du talus est constituée d'une ou plusieurs couches parallèles à la surface du talus, il est nécessaire d'effectuer une vérification de la stabilité de l'ouvrage vis-à-vis d'un glissement de la protection du talus sur une surface de glissement superficielle et plane. En effet, ces interfaces séparant les couches peuvent présenter des caractéristiques plus faibles que les matériaux constituant chacune des couches. Par ailleurs, il peut se produire un glissement le long de l'une des interfaces lorsque les pressions interstitielles au niveau de ces interfaces sont supérieures aux pressions hydrauliques au niveau de la surface du talus (notamment en cas de vidange rapide). Ce phénomène peut notamment se produire au niveau de l'interface séparant une couche relativement perméable et une carapace relativement imperméable. 
L'analyse du glissement de la protection du talus sous l'effet des vagues levées par le vent ou la navigation doit couvrir les aspects suivants :

- l'estimation des niveaux extrêmes atteints par les vagues ou le batillage (cas des rivières navigables) ;

- l'estimation de la pression interstitielle juste en dessous de chaque interface, qui peut être influencée par la surélévation du niveau piézométrique interne ;

- l'estimation de l'angle de frottement de l'interface ;

- l'estimation de la résistance de l'ensemble des couches ou des matériaux de remplissage supportant d'autres matériaux.

\section{4}

\section{État limite de défaut de portance de la fondation/tassements}

Pour le cas des fondations compressibles, il convient de vérifier la digue vis-à-vis de l'état limite de défaut de capacité portante de la fondation (poinçonnement). La situation à envisager est la fin de construction du remblai.

Dans le calcul, seule la résistance au cisaillement de la couche la plus médiocre de la fondation est prise en compte (cette couche doit avoir été mise en évidence lors des reconnaissances) ; en effet, le remblai (s'il a été compacté) est en général nettement plus résistant que cette dernière.

Dans le cas d'une couche peu résistante mince, le calcul en rupture non circulaire est pertinent. Toutefois, en prenant une épaisseur de fondation meuble peu résistante d'au moins $\mathrm{H} / 3$, $\mathrm{H}$ étant la hauteur de la digue, on obtient en général des résultats relativement proches pour les trois types de ruptures suivantes : non circulaire, circulaire et au poinçonnement.

En ce qui concerne l'état limite de déformations (ELS), une estimation des déformations et des tassements peut être nécessaire lorsqu'on se propose de mettre en place la digue :

- sur des matériaux compressibles (argile par exemple) ;

- aux abords immédiats d'ouvrages sensibles aux tassements différentiels, ou d'ouvrages fondés sur des pieux.

Les digues construites sur des fondations granulaires, pour lesquelles les tassements et déformations ne sont pas préjudiciables à l'ouvrage, ne font pas l'objet d'une vérification vis-à-vis de cet état limite.

Rappelons cependant que, pour ces ouvrages à grand linéaire, la surveillance des tassements est dans tous les cas essentielle afin de vérifier le maintien en tout point de la revanche entre la ligne d'eau et la crête.

\section{5}

\section{États limites d'affouillement et d'érosion de berge de canal}

\section{6}

Définitions

On distingue l'affouillement de pied, qui est susceptible d'entraîner la rupture générale du talus (ELU), et l'érosion le long du talus, qui est un phénomène plus local et progressif (ELS).

1) L'affouillement en pied de talus : C'est un phénomène soit naturel, soit lié à l'influence d'ouvrages qui perturbent l'écoulement. L'affouillement est très courant lorsque les sédiments sont érodables, qu'il s'agisse de matériaux fins (limon ou sable) ou grossiers (galets, graviers). Selon son étendue spatiale, l'affouillement peut entraîner un approfondissement généralisé du fond ou être à l'origine de fosses d'affouillement localisées. La formation de fosses d'affouillement à proximité de la digue peut entraîner une rupture géotechnique sous forme de grand glissement. Par ailleurs, l'augmentation des profondeurs d'eau due au phénomène d'affouillement généralisé peut entraîner un accroissement de l'action hydraulique (guide Enrochement, 2009).

2) L'érosion en partie intermédiaire de talus : la dégradation du parement amont est consécutive aux sollicitations liées aux vagues (dues aux vents, aux bateaux). Elle peut, à la longue, entraîner une rupture locale (soutirage des matériaux fins par marnages successifs, glissement, raidissement graduel de la pente). Ce type de rupture peut être prévenu par une surveillance et une maintenance adaptées.

\section{6}

\section{Types de sollicitations}

Les sollicitations liées à l'eau ont trois origines principales (CETMEF, juillet 2009) :

- le passage des bateaux (batillage, courants de retour, déferlement, jets d'hélice...) ;

- les fluctuations du niveau de l'eau (fonctionnement des écluses, abaissement pour chômage, différences piézométriques avec une nappe de versant...);

- les phénomènes naturels (cas des rivières) tels que les courants naturels, les crues, les évolutions morphologiques dans les méandres (répartition non uniforme des vitesses et surélévation de la surface libre du côté de la rive concave), etc.

Quelle que soit leur origine, quatre natures de sollicitations se distinguent :

- les courants (courants naturels ou induits par la navigation...) ;

- les vagues (vagues de vent ou de batillage...) ;

- l'abaissement du plan d'eau (marnage, abaissement du plan d'eau dû à la navigation...) ou son exhaussement (crues, onde de proue...) ;

- l'arrivée d'eau par le sol (apports d'eaux souterraines).

Les courants et les vagues peuvent provoquer une érosion des matériaux de la berge et du lit, alors qu'un 
abaissement rapide du plan d'eau ou l'arrivée d'eau par le sol peuvent produire des glissements ou des affaissements de terrain.

Les sollicitations liées aux courants naturels, aux vagues induites par le vent et les sollicitations liées à la navigation sont décrites en annexe 4, ainsi que la façon de les quantifier. La zone la plus affectée par les courants naturels est le pied de la berge.

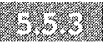

\section{Affouillement en pied de talus (ELU)}

La justification de la digue vis-à-vis de l'état limite d'affouillement consiste à vérifier la stabilité de la berge en tenant compte :

1) des sollicitations hydrauliques (dimensionnement hydraulique). Dans ce cas, la démarche comporte les étapes suivantes :

- identification et détermination des sollicitations hydrauliques ( $\$ 5.5 .2$ ),

- en cas de berge naturelle non protégée, vérification de la résistance à l'érosion du sol vis-à-vis des sollicitations hydrauliques,

- en cas de berge protégée avec des enrochements ou matelas gabions, vérification de la protection vis-à-vis des sollicitations hydrauliques,

- définition de la profondeur d'affouillement;

2) des conditions de stabilité géotechnique (dimensionnement géotechnique). Dans ce cas, la démarche consiste à analyser la stabilité du talus de la berge ou de la berge protégée par rapport aux mécanismes suivants :

- rupture par glissement circulaire,

- rupture par glissement plan,

- rupture par défaut de capacité portante,

- tassements, déformations.

\section{Whe}

Érosion en partie intermédiaire de talus (ELS)

\section{6.}

\section{Résistance à l'érosion dés sols}

Une première approximation de la résistance à l'érosion des différents sols est donnée ci-après.

- Matériaux granulaires (sable et galets)

La méthode pratique dans le cas de la stabilité des sédiments non cohésifs allant du sable aux galets de taille moyenne $(62 \mu \mathrm{m}<\mathrm{D}<8 \mathrm{~mm})$ est la méthode de la contrainte de cisaillement basée sur le critère de Shields. Il est à noter qu'il faut prêter attention à la rugosité hydraulique, $\mathrm{k}_{\mathrm{s}^{\prime}}$ lors de la détermination $\mathrm{du}$ coefficient de cisaillement au fond.

\section{- Sédiments cohésifs}

L'interaction physico-chimique entre les particules joue un rôle important dans la résistance hydraulique (érodabilité) des sédiments cohésifs. Pour l'instant, la détermination de la vitesse critique repose encore large- ment sur des données empiriques issues de différentes expériences et observations in situ. Les connaissances dont on dispose actuellement sur la corrélation entre $\psi_{c r}$ (facteur de stabilité de type Shields) et/ou la vitesse du courant critique, $\mathrm{U}_{c r}$ la nature des sols (teneur en limon, en argile, indice de plasticité...), leurs états (densité, états hydriques) ou leurs caractéristiques mécaniques (cohésion, angle de frottement, etc.) sont encore insuffisantes pour permettre de déterminer une approche générale. Les matériaux cohésifs tels que l'argile présentent habituellement une résistance à l'érosion supérieure à celle des matériaux non cohésifs.

A titre indicatif, les valeurs de $\mathrm{U}_{\mathrm{cr}}$ suivantes peuvent être utilisées :

- argile assez compacte (indice des vides e $=0,5$ ) : $\mathrm{U}_{\mathrm{cr}}=0,8 \mathrm{~m} / \mathrm{s}$;

- argile rigide (indice des vides $e=0,25$ ) : $U_{c r}=1,5 \mathrm{~m} / \mathrm{s}$;

- argile recouverte d'herbe : $U_{c r}=2 \mathrm{~m} / \mathrm{s}$;

- berges en argile recouvertes d'herbe (conçues et entretenues de manière appropriée et/ou renforcées avec des matelas géotextiles tridimensionnels) : $U_{c r}$ jusqu'à $3 \mathrm{~m} / \mathrm{s}$.

\section{1.}

\section{Justification des protections de berge}

Dans la pratique, différents types de revêtements sont utilisés, par exemple, le béton bitumineux, les palplanches, les matelas de gabions ou les enrochements. Dans ce document, nous nous focalisons uniquement sur le cas des berges protégées par un revêtement constitué d'enrochements libres ou de matelas de gabions, en abordant également les protections végétales.

En effet, dans le cas d'une berge protégée par un revêtement en béton de ciment, en béton bitumineux ou par des palplanches métalliques, le batillage n'intervient dans le calcul de ces protections qu'en raison des sous-pressions qu'il peut provoquer. Ces sous-pressions sont relativement faibles et ne sont en général pas déterminantes pour le dimensionnement de ces ouvrages (Tenaud, 24e congrès de l'AIPCN, 1977).

Pour la justification des protections, il est d'usage de considérer deux situations :

- berges en alignement droit ;

- berges en courbe.

\section{Whe}

\section{Protections de berges en enrochements}

La méthodologie, détaillée en annexe 5, consiste essentiellement à déterminer les éléments de dimensionnement suivants :

1) dimensions maximales de la protection : profondeur, hauteur le long du talus et largeur de la banquette (dans le cas où elle est nécessaire) ;

2) dimension moyenne des blocs et épaisseur de la protection;

3) introduction des corrections relatives à la pente, la courbure, la sinuosité et la densité des blocs ;

4) blocométrie (courbe granulométrique) ;

5) couche filtre. 
Une protection adéquate de pied d'un talus ou d'une berge est essentielle pour sa stabilité, dans la mesure où de nombreux mécanismes de rupture résultent d'une perte de résistance à la base du talus. Si la protection du fond et des berges n'est pas continue, il existe deux façons de garantir la protection du pied:

- en plaçant assez de matériaux à une profondeur suffisante pour prendre en considération la profondeur d'affouillement maximale prévue ;

- en installant un revêtement flexible (banquette) qui continuera à protéger le pied pendant l'évolution de la fosse d'affouillement.

Au vu de ce qui précède, il est évident que l'estimation de l'affouillement peut constituer une étape importante dans le dimensionnement d'ouvrages en enrochement stables.

\subsection{4 .4}

\section{Protections de berges en matelas de gabions}

On peut se référer à la norme NF-P 94-325-2 et au guide enrochement (principalement section 8.6.2), dont les principaux éléments sont repris en annexe 6 du présent document.

\section{5}

Protection anti-érosion par techniques végétales

Ce point fait l'objet de l'annexe $7 \mathrm{du}$ présent document.

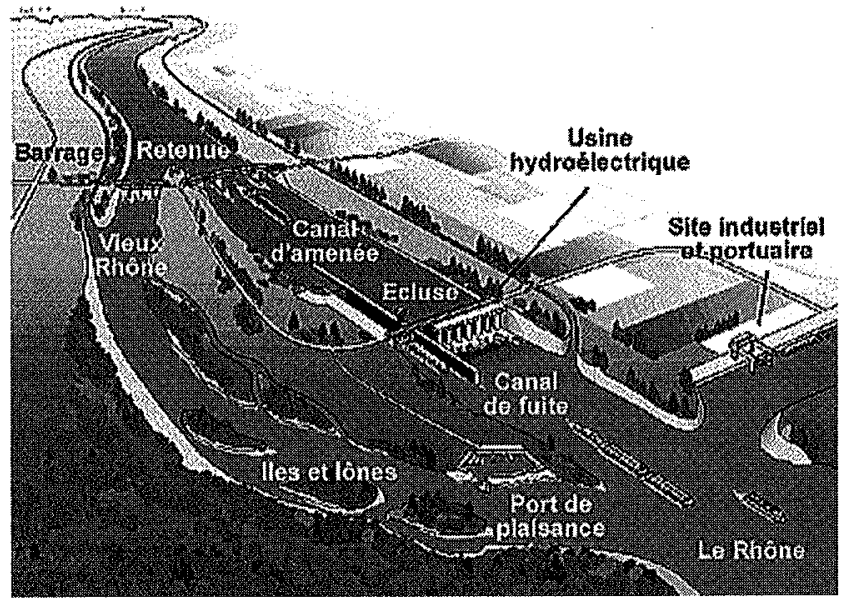

Ecs.s1: L'aménagement type du Rhône.

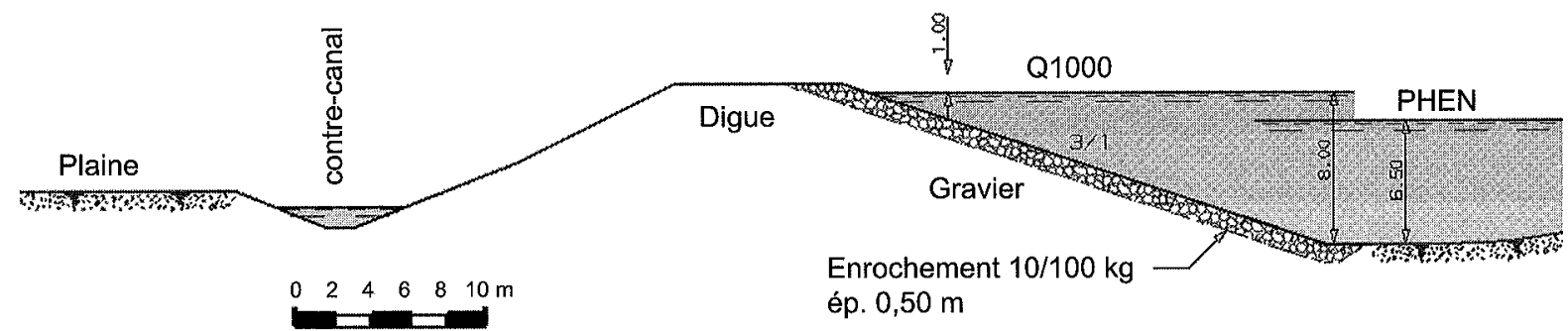

ép. $0,50 \mathrm{~m}$

Flc. 5.2. Coupe type d'une digue (sollicitations hydrauliques normales)

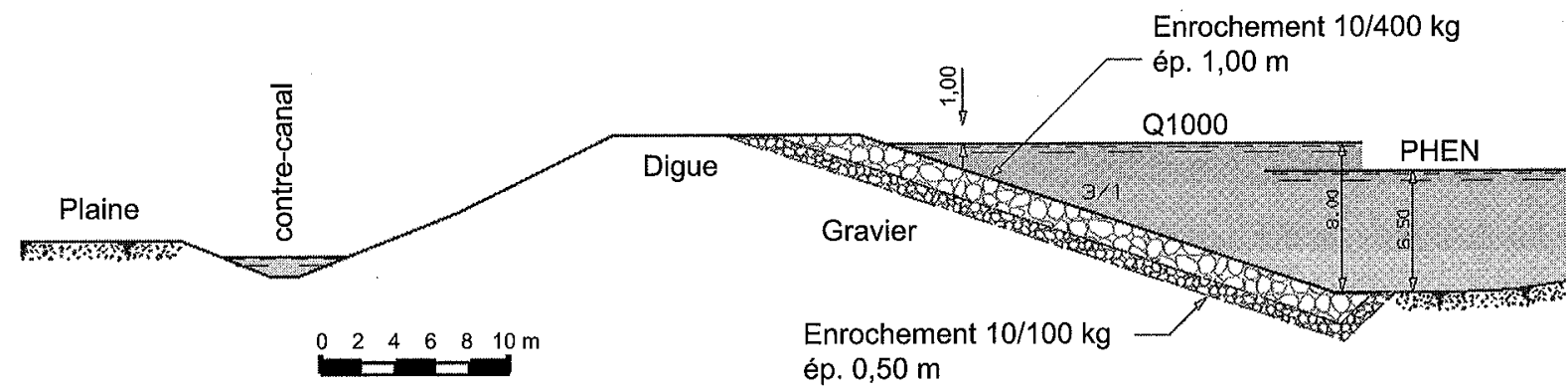

FI6. 5.8 Coupe type d'une digue (sollicitations hydrauliques fortes)

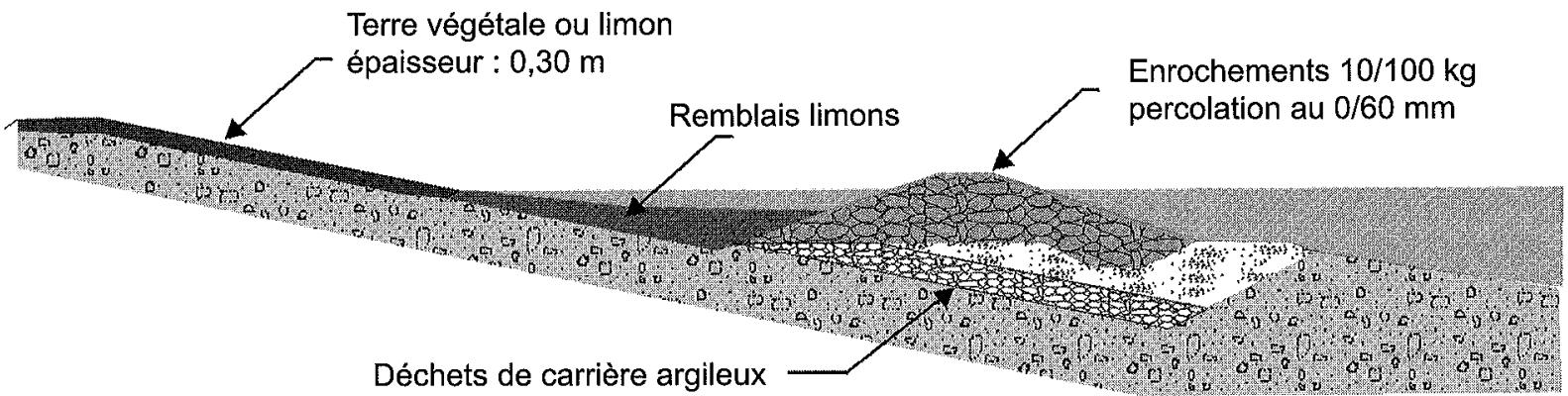

116. 5.4 Exemple de protection de talus avec végétation. 


\section{Justification de la sécurité des digues de protection contre les Inondations}

\section{6.,}

\section{Généralités}

Pour les digues à charge permanente ou non, on appelle amont le côté rivière et aval le côté terres (ou coté val). Amont et aval s'entendent ici sur une coupe en travers de la digue et non le long du cours d'eau.

Les dispositions de cette partie s'appliquent aux digues de protection contres les inondations fluviales, aux digues de rivières canalisées, aux digues qui ceinturent des lieux habités. Il s'agit d'ouvrages qui se trouvent essentiellement le long des cours d'eau et qui sont destinés à contenir épisodiquement un flux d'eau ou une charge hydraulique afin de protéger des zones naturellement inondables. Ces ouvrages, considérés comme des digues au sens de la nouvelle réglementation sur les ouvrages hydrauliques (décret du 11 décembre 2007), se distinguent principalement des barrages en remblai par leurs dimensions (hauteur généralement plus faible et longueur beaucoup plus importante), leurs conditions aux limites (sollicitation hydraulique principalement transitoire, écoulement longitudinal), leur conception (absence de dispositif d'étanchéité ou de drainage le plus souvent), et même souvent par leur absence de conception, étant édifiés comme des cordons de curage puis engraissées au fil des générations.

Les états limites ultimes qu'il convient de considérer pour ces digues sont:

- l'état limite ultime de stabilité d'ensemble (glissement) ;

- l'état limite ultime de soulèvement hydraulique au pied aval ;

- l'état limite ultime de défaut de portance (poinçonnement de la fondation) ;

- l'état limite de service de tassement ;

- l'état limite ultime d'affouillement en pied amont ;

- les états limites de boulance, de résistance à l'érosion interne et de résistance à la surverse (traités ultérieurement) ;

- l'état limite de service de filtration (traité ultérieurement);

- l'état limite de liquéfaction sous sollicitation sismique (traité au sein d'un autre GT).

Pour les états limites de stabilité d'ensemble, de soulèvement du sol et de défaut de portance de la fondation, seules les spécificités des digues non soumises à une charge permanente sont traitées dans ce chapitre et il convient de se référer au paragraphe 4 « Justification de la sécurité des barrages en remblai » pour les dispositions communes avec les barrages en remblai. Les états limites de boulance, d'érosion interne et de surverse seront traités dans une autre publication.
Pour les situations de projet, il convient de se référer à la partie 1 de ces recommandations.

Pour les données géotechniques, il convient de se référer aux indications de la partie 2 de ces recommandations. Pour les digues existantes, il est souvent difficile d'avoir une vision claire de la constitution des ouvrages et de leurs fondations.

Avant de procéder à des modélisations hydrauliques complexes (sur la base de caractéristiques rarement mesurées), la priorité doit être donnée à la connaissance réelle des matériaux (contrastes des perméabilités, anisotropie) et à l'instrumentation de l'ouvrage. Il convient de s'intéresser aux données quantitatives simples (granulométrie, indice de plasticité, densité) et aux essais in situ.

Avant de procéder à des calculs aux états limites, il est recommandé de procéder à une analyse exhaustive des informations se rapportant à l'historique de l'ouvrage, son fonctionnement, sa constitution, et aux éventuels modes de rupture passés. L'effet sur la digue de la végétation, des galeries creusées par les animaux fouisseurs et de points singuliers (conduites traversantes, raccordement à des ouvrages de génie civil...) est évalué dans un premier temps de manière qualitative.

\section{2}

\section{Situations et états limites à considérer}

Le tableau 6.I présente les situations de projet à analyser, ainsi que les états limites correspondants (hors érosion interne).

\section{État limite de stabilité d'ensemble (glissement)}

La méthode de justification de la stabilité des digues en terre ou en matériaux rocheux vis-à-vis de l'état limite de glissement est identique à celle utilisée pour les barrages en remblai. Cette méthode s'applique à la stabilité interne de l'ouvrage (stabilité de ses talus, notamment) et à sa stabilité d'ensemble (ou globale) lorsqu'il est situé sur ou près d'une pente et risque d'être déplacé par un glissement de terrain. Les surfaces de glissement peuvent avoir n'importe quelle forme, mais les analyses prennent généralement pour hypothèse une forme circulaire ou plane. L'approche la plus couramment utilisée et recommandée est la méthode de Bishop, qui nécessite l'utilisation de programmes informatiques.

En alternative à la méthode des coefficients partiels, une étude de sensibilité adaptée à l'état limite de grand glissement (glissement généralisé) peut être effectuée (ROSA 2000).

L'hypothèse de régime permanent en situation de crue est souvent pessimiste pour la stabilité du talus aval. Les calculs en régime transitoire sont alors intéressants car ils mettent en évidence que le corps de digue n'a, le plus souvent, pas le temps de se saturer pendant la durée de la crue. Ceci dépend bien évidem- 


\section{SITUATIONS NORMALES D’EXPLOITATION}

Situation normale d'exploitation
Niveau moyen de la rivière hors crue

Écoulement permanent établi Caractéristiques mécaniques à long terme
Établissement du régime permanent de piézométrie dans le corps de la digue. La piézométrie est imposée par le niveau de la rivière et éventuellement la nappe

Glissement du talus aval Glissement du talus amon Affouillement pied amont

\section{SITUATIONS TRANSITOIRES OU RARES}

Situation transitoire de fin de construction

Situation rare d'étiage ou d'érosion de pied

Situation rare de crue

Situation transitoire de décrue rapide
Pour ouvrages neufs ou ouvrages existants avec rechargement (ou baisse prolongée de la nappe)

Caractéristiques mécaniques à court terme

Niveau d'étiage le plus fort de la rivière (périodicité 10 à 100 ans)

Érosion de pied si tendance suspectée

Caractéristiques mécaniques à long terme

Niveau de la cote de protection (cote du déversoir de sécurité d'une digue ou cote de la digue aménagée pour résister à la surverse)

Périodicité $=10$ à 100 ans

Depuis le niveau de crue rare de la rivière (cote de protection)
Pour les remblais argileux ou sur sol compressible, cette situation peut correspondre à des pressions interstitielles, qui se seraient développées pendant la construction et ne se seraient pas dissipées

Abaissement rare de la ligne d'eau après que le régime permanent ait été établi

Glissement du talus amon Affouillement pied amont

talus amont

Glissement du talus aval

Défaut de portance de la

fondation
Élévation de la ligne d'eau après que le régime permanent a été établi. Régime transitoire car les pressions interstitielles n’ont pas nécessairement le temps de s'établir

Abaissement rapide de la ligne d'eau avec un niveau piézométrique maintenu dans la digue au niveau de la crue considérée

\section{SITUATIONS EXCEPTIONNELLES}

Situation exceptionnelle de crue
Cote des plus hautes eaux ou cote de la rivière, laissant encore une revanche pour se protéger de l'effet des vagues

Périodicité $=50$ à 1000 ans selon les risques

\section{SITUATIONS ACCIDENTELLES}

Situation (accidentelle) de crue ou situation de la crue de danger

Débit de crue de plein bord ou cote au-delà de laquelle l'ouvrage risque de subir des dégâts majeurs pouvant conduire rapidement à la rupture

Périodicité supérieure à la précédente

Situation accidentelle de séisme (SES)
On considère que cette situation ne peut pas être concomitante d'une crue
Effet des efforts d'inertie, et surtout, dans certains cas, augmentation des pressions interstitielles
Glissement du talus aval

Soulèvement pied aval

Affouillement pied amont
Glissement du talus aval Affoullement pied amont Soulèvement pied aval

Glissement du talus amon

\section{STTUATIONS LIÉES A LA DÉFAILLANCE D’UN COMPOSANT}

Situation (rare ou accidentelle) de défaillance du contrôle des écoulements

Situation (transitoire) de travaux en pied de digue
On cherche ici les scénarios (rares ou accidentels selon leur probabilité d'occurrence), tenant compte des dispositions d'auscultation et d'entretien adoptées.

Il peut s'agir d'une rupture ou défaillance lors d'événements extérieurs (séisme, malveillance) ou liées au vieillissement

Situation à éviter, voire à proscrire
Les risques d'altération ou de rupture des dispositifs d'étanchéité et de drainage sont évalués (dégradation d'un rideau, voile ou masque d'étanchéité, colmatage d'un drain...)

Par exemple creusement d'une tranchée en pied de digue
Glissement du talus aval Soulèvement pied aval Affouillement pied amont

Glissement des talus (surtout aval)

Liquéfaction

Glissement du talus amon Glissement du talus aval Soulèvement pied aval. Affouillement pied amont

Glissement du talus aval. Défaut de portance de la fondation 
ment de la nature et donc de la perméabilité des matériaux constituant la digue et son support.

Cependant, pour le calcul des pressions interstitielles en situation de crue, les modèles actuels ne permettent pas de mener et de vérifier les calculs en régime transitoire de manière rigoureuse. En effet, dans le cas des digues de protection contre les inondations, les données manquent en général pour faire un calcul réaliste en régime transitoire (il faut des courbes $\mathrm{k}-\mathrm{S}_{\mathrm{r}}$ et $\mathrm{w}-\mathrm{S}_{\mathrm{r}^{\prime}}$ perméabilité et teneur en eau en fonction de la saturation, dont on dispose rarement) et les résultats obtenus à partir des modèles sont alors douteux dès qu'il est fait l'hypothèse d'un sol non saturé. Il est aussi à noter que les forts contrastes de perméabilité entre matériaux peuvent provoquer des instabilités de logiciels.

Pour le calcul en décrue, on peut généralement considérer que la ligne de saturation est horizontale (niveau de la cote de protection) dans la zone étanche de la digue (talus amont ou zone centrale). Cette approximation n'affecte pas sensiblement le résultat du calcul de stabilité, qui est également peu affecté par une variation du poids volumique des matériaux.

Pour la vérification des digues existantes vis-à-vis de l'état limite de glissement, il est possible de s'appuyer sur le fait que la digue est toujours en place. Cette information peut être valorisée de deux manières:

- en comparant la section la plus défavorable aux autres sections, des facteurs de sécurité sont alors calculés pour les autres sections ;

- en calculant un gain sur le facteur de sécurité (plutôt que de viser les valeurs recommandées) si un renforcement est envisagé. Selon le cas, on pourra rechercher un gain de $+20 \%$ à $+30 \%$.

\section{7}

\section{État limite de soulèvement hydraulique en pied aval}

L'instabilité du pied aval peut être initiée, non pas par des écoulements, mais par l'apparition de pressions d'eau dans le cas des digues reposant sur une couche mince imperméable reposant elle-même sur une couche de forte perméabilité (alluvions).

Ce cas est particulièrement fréquent pour les digues de protection contre les inondations, construites sur une fondation alluviale qui comporte souvent des alternances de couches de perméabilité contrastée (cf. $\S 4.5)$.

\section{5}

\section{État limite de défaut de capacité portante de la fondation/tassements}

Pour le cas des fondations compressibles, il convient de vérifier la digue vis-à-vis de l'état limite de défaut de capacité portante de la fondation (poinçonnement). La situation à envisager est la fin de construction du remblai.
Dans le calcul, seule la résistance au cisaillement de la couche la plus médiocre de la fondation est prise en compte (cette couche doit avoir été mise en évidence lors des reconnaissances); en effet, le remblai (s'il a été compacté) est en général nettement plus résistant que cette dernière.

Dans le cas d'une couche peu résistante mince, le calcul en rupture non circulaire est pertinent. Toutefois, en prenant une épaisseur de fondation meuble peu résistante d'au moins $\mathrm{H} / 3, \mathrm{H}$ étant la hauteur de la digue, on obtient en général des résultats relativement proches pour les trois types de ruptures suivantes: non circulaire, circulaire et au poinçonnement.

En ce qui concerne l'état limite de déformations (ELS), une estimation des déformations et des tassements peut être nécessaire lorsque la digue est construite :

- sur des matériaux compressibles (argile par exemple) ;

- aux abords immédiats d'ouvrages sensibles aux tassements différentiels, ou d'ouvrages fondés sur des pieux.

Les digues construites sur des fondations granulaires, pour lesquelles les tassements et déformations ne sont pas préjudiciables à l'ouvrage, ne font pas l'objet d'une vérification vis-à-vis de cet état limite.

Rappelons cependant que, pour ces ouvrages à grand linéaire, la surveillance des tassements est dans tous les cas essentielle afin de vérifier le maintien en tout point de la revanche entre les lignes d'eau en crue et la crête.

\section{6 \\ État limite d'affouillement}

Il est provoqué par les sollicitations liées à l'eau, en régime d'étiage et lors des crues, et ayant pour origine :

- les courants de la rivière ;

- les évolutions morphologiques dans les méandres (cf. § 2.1);

Pour les justifications, on se reporte au chapitre 5.5.4, en ne considérant que les aspects liés aux courants naturels.

\section{Annexes}

\section{- A1 \\ Valeurs standard pour les paramètres géotechniques}

Les valeurs données ci-dessous proviennent de corrélations et de compilations publiées dans la littérature. Il ne s'agit pas nécessairement de valeurs caractéristiques, mais plutôt de valeurs moyennes (extrait du cours de géotechnique de Léreau - INSA Toulouse). 


\section{Caractéristiques physiques}

\begin{tabular}{|c|c|c|}
\hline$\gamma$ & 18 kN/m³ & 10 à $20 \mathrm{kN} / \mathrm{m}^{3}$ \\
\hline$\gamma \mathrm{d}$ & 14 à $18 \mathrm{kN} / \mathrm{m}^{3}$ & 10 à $20 \mathrm{kN} / \mathrm{m}^{3}$ \\
\hline ysat & 19 à $22 \mathrm{kN} / \mathrm{m}^{3}$ & 19 à $22 \mathrm{kN} / \mathrm{m}^{3}$ \\
\hline$\gamma \mathrm{s}$ & 26 à $30 \mathrm{kN} / \mathrm{m}^{3}$ & 26 à $30 \mathrm{kN} / \mathrm{m}^{3}$ \\
\hline$\gamma$ & 9 à $12 \mathrm{kN} / \mathrm{m}^{3}$ & 9 à $12 \mathrm{kN} / \mathrm{m}^{3}$ \\
\hline n & 0,25 à 0,50 & 0,20 à 0,80 \\
\hline e & 0,50 à 1 & 0,30 à 1 \\
\hline w & 1 à $15 \%$ & 10 à $20 \%$ \\
\hline
\end{tabular}

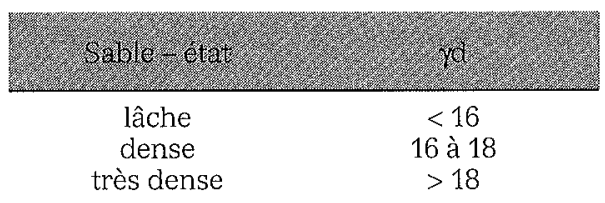

\section{Cohésion et angle de frottement interne} tiques

Enrochements, graviers, sables et limons non plas-

Cohésion :

$-\mathrm{c}^{\prime}=0$

Angle de frottement interne :

- enrochements $\varphi^{\prime}=40-45^{\circ}$
- gravier sableux, sable ou gravier à granulométrie étalée $\varphi^{\prime}=30-35^{\circ}$, voire $40^{\circ}$

- sable à granulométrie uniforme, limon non plastique $\varphi^{\prime}=20-30^{\circ}$

Influence des caractéristiques physiques sur l'angle de frottement interne d'un matériau pulvérulent

\begin{tabular}{|c|c|c|c|}
\hline Compacité & $\varphi_{1}^{\prime}$ & $\begin{array}{r}-6^{\circ} \\
0^{\circ} \\
+6^{\circ} \\
\end{array}$ & $\begin{array}{l}\text { lâche } I_{D}=0,4 \\
\text { moyen } I_{D}=0,6 \\
\text { serré } I_{D}=0,9\end{array}$ \\
\hline $\begin{array}{c}\text { Forme et rugosité } \\
\text { des grains }\end{array}$ & $\varphi_{2}^{\prime}$ & $\begin{array}{l}+1^{\circ} \\
0^{\circ} \\
-3^{\circ} \\
-5^{\circ} \\
\end{array}$ & $\begin{array}{l}\text { aigu } \\
\text { moyen } \\
\text { arrondi } \\
\text { sphérique }\end{array}$ \\
\hline Grosseur des grains & $\varphi_{3}^{\prime}$ & $\begin{array}{r}0^{\circ} \\
+1^{\circ} \\
+2^{\circ} \\
\end{array}$ & $\begin{array}{l}\text { sable fin } 0,06 \mathrm{~mm}<\mathrm{D}_{10}<0,2 \mathrm{~mm} \\
\text { gravier } 0,6 \mathrm{~mm}<\mathrm{D}_{10}<2 \mathrm{~mm} \\
\text { gros gravier } \mathrm{D}_{10}>2 \mathrm{~mm}\end{array}$ \\
\hline $\begin{array}{l}\text { Répartition } \\
\text { granulaire }\end{array}$ & $\varphi_{4}^{\prime}$ & $\begin{array}{r}-3^{\circ} \\
0^{\circ} \\
+3^{\circ}\end{array}$ & $\begin{array}{l}\text { uniforme } c_{u} \leq 2 \\
\text { moyenne } 2<c_{u}<5 \\
\text { étalée } c_{u} \geq 5\end{array}$ \\
\hline
\end{tabular}

Relation de Kerisel pour les sables : e $\cdot \tan \varphi^{\prime}=$ cste $=0,55$.

Argile saturée. Le drainage s'effectue lentement.

Cohésion :

$-\mathrm{C}_{u}=$ de quelques dizaines à quelques centaines de $\mathrm{kPa}$

$-C^{\prime}$ négligeable

Angle de frottement interne:

$-\varphi_{u}=0$

$-\varphi^{\prime}: 10-20^{\circ}$, quelquefois plus.

Argile humide non saturée. Les propriétés mécaniques avant et après drainage sont du même ordre.

Cohésion :

$-c_{u}$ et $c^{\prime}$ de quelques dizaines à quelques centaines de $\mathrm{kPa}$.

Angle de frottement interne :

$-\varphi_{u}$ et $\varphi^{\prime}$ de quelques degrés à une vingtaine, quelquefois plus.
Argile sèche $\left(\mathrm{S}_{\mathrm{r}}<0,7\right)$. Il ne se produit pas de drainage et il n'y a aucune différence entre les propriétés du sol mesurées dans un essai drainé ou non drainé.

Cohésion :

$-c_{u}=c^{\prime}$, quelques centaines de $\mathrm{kPa}$ (susceptible de beaucoup diminuer en cas d'humidification).

Angle de frottement interne :

$-\varphi_{u}=\varphi^{\prime}$ supérieur à $10^{\circ}$.

Cohésion non drainée $c_{u}$ pour quelques consistances types d'argile.

\begin{tabular}{ll}
\hline & \\
\hline
\end{tabular}


Exemples de corrélation (source : Philipponat)

Corrélations entre pressiomètre et nombre N du SPT.

\begin{tabular}{lll} 
Nature disoli & El (MPa) \\
\hline Limon & N/30 à 35 & $\mathrm{~N} / 2,5$ à 3,0 \\
Sable & $\mathrm{N} / 15$ à 25 & $\mathrm{~N} / 1,5$ à 3,0 \\
Argile & $\mathrm{N} / 15$ à 30 & $\mathrm{~N} / 1,0$ à 2,5 \\
Marne & $\mathrm{N} / 20$ à 25 & $\mathrm{~N} / 1,5$ à 2,5 \\
Craie & $\mathrm{N} / 5$ à 20 & $\mathrm{~N} / 0,7$ à 1,0 \\
\hline
\end{tabular}

Rapport entre $\mathrm{q}_{\mathrm{d}}$ et $\mathrm{q}_{\mathrm{c}^{\prime}} \mathrm{p}_{\mathrm{r}^{\prime}} \mathrm{E}_{\mathrm{M}}$.

\begin{tabular}{|c|c|c|c|}
\hline $\begin{array}{l}\text { Argiles, limons et vases } \\
\text { normalement consolidés, } \\
\text { sables lâches ou } \\
\text { moyennement denses }\end{array}$ & $\approx 1$ & 1,4 à 2,5 & 0,1 à 0,3 \\
\hline $\begin{array}{l}\text { Argiles et limons } \\
\text { surconsolidés }\end{array}$ & 1 à 2 & 3 à 5 & 0,2 à 0,4 \\
\hline $\begin{array}{l}\text { Sables et graviers, sables } \\
\text { limoneux ou argileux denses } \\
\text { à très denses }\end{array}$ & 0,5 à 1 & 5 à 10 & 0,4 à 1,5 \\
\hline
\end{tabular}

Rapport entre $\mathrm{q}_{\mathrm{c}}$ et le module œdométrique $\mathrm{E}_{\text {od' }}$ selon G. Sanglerat.

\begin{tabular}{|c|c|c|}
\hline Argile peu plastique & $\begin{array}{c}<0,7 \\
0,7 \text { à } 2 \\
>2\end{array}$ & $\begin{array}{c}3 \text { à } 8 \\
2 \text { à } 5 \\
1 \text { à } 2,5\end{array}$ \\
\hline Limon peu plastique & $\begin{array}{l}<2 \\
>2\end{array}$ & $\begin{array}{l}3 \text { à } 6 \\
1 \text { à } 3\end{array}$ \\
\hline Argile et limon très plastiques & $<2$ & 2 à 6 \\
\hline Limon fortement organique & $<1,2$ & 2 à 8 \\
\hline $\begin{array}{l}\text { Tourbe et argile organique } \\
50<w<100 \\
100<w<200 \\
200<w\end{array}$ & $<0,7$ & $\begin{array}{l}1,5 \text { à } 4 \\
1 \text { à } 1,5 \\
0,4 \text { à } 1\end{array}$ \\
\hline Craie molle & 2 à 3 & 2 à 4 \\
\hline Sable & $\begin{array}{c}<5 \\
>10\end{array}$ & $\begin{array}{c}2 \\
1,5\end{array}$ \\
\hline
\end{tabular}

\section{Coefficient de Poisson}

On indique des valeurs du coefficient de Poisson en fonction de la nature des sols et de leur état:

- sols granulaires :

- sols lâches : $v=0,20$,

- sols denses : $v=0,33$;

- sols fins :

- pour le cas des déformations instantanées (comportement non drainé, sols saturés, donc volume constant) : $v=0,50$;

- pour le cas des déformations totales (comportement drainé) :

- sols normalement consolidés : $v=0,40$,

- sols surconsolidés, et si l'on reste loin de la rupture : $v=0,25$ à 0,35 .

\section{Hydraulique des milieux poreux : principaux concepts et formules}

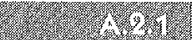 \\ Définitions}

Le potentiel hydraulique en un point, appelé aussi charge hydraulique, est l'énergie de l'eau en ce point. Cette énergie est la somme d'une énergie potentielle, d'une énergie de pression et d'une énergie cinétique. Dans les écoulements souterrains, le terme d'énergie cinétique est très souvent négligeable devant la pression et l'énergie potentielle. Le potentiel hydraulique est de ce fait généralement exprimé dans la dimension d'une hauteur (en mètres) et se dénomme alors hauteur piézométrique, égale à la somme de l'altitude du point considéré, $z$, et de la hauteur de la colonne d'eau appliquant la pression d'eau $\mathrm{p}_{\mathrm{w}}, \mathrm{p}_{\mathrm{w}} / \gamma_{\mathrm{w}}$ (la pression d'eau est comptée à partir de la pression atmosphérique en considérant $\mathrm{p}_{\mathrm{atm}}=0$ ) :

$$
\mathrm{H}=\mathrm{z}+\mathrm{p}_{\mathrm{w}} / \gamma_{\mathrm{w}}
$$

La succion totale, $\psi(m)$, est la succion totale, somme de la succion matricielle et la succion osmotique. Représentant l'énergie à apporter à un gramme d'eau dans un sol non saturé pour rendre l'eau libre, elle remplace donc le terme $\mathrm{p}_{w} / \gamma_{w}$ dans l'expression du potentiel hydraulique avec un signe négatif. La pression d'eau a une valeur négative dans un sol non saturé à l'équilibre avec l'atmosphère, donc $\left(p_{w}-p_{a}\right)<0$. Pour des raisons de commodité, le signe de la succion est pris positif.

$$
\mathrm{H}=\mathrm{z}-\psi
$$

La succion matricielle, $\mathrm{s}$ (Pascal), est la valeur absolue de la différence entre la pression d'eau et la pression d'air dans le sol non saturé. Il importe de savoir que la succion matricielle, plus facile à mesurer que la succion totale, la remplace souvent dans les calculs. D'autre part, en cours de construction, l'air est comprimé, et si son écoulement est lent, sa pression devient supérieure à la pression atmosphérique.

La porosité, $n$, est le rapport du volume des vides sur le volume total.

Le degré de saturation, $\mathrm{S}_{\mathrm{r}}$ est le rapport de la teneur en eau sur la teneur en eau de saturation.

Le tenseur de conductivité hydraulique, $\mathrm{k}_{\mathrm{ij}}(\mathrm{m} / \mathrm{s})$, dont les 9 composants sont les coefficients de proportionnalité entre les 3 projections sur 3 plans orthogonaux de la vitesse d'infiltration et du gradient de charge hydraulique. Les composantes principales sont appelées coefficients de perméabilité.

Le coefficient d'emmagasinement, C $(1 / \mathrm{m})$, est la variation relative de volume d'eau stockée dans un volume unitaire de terrain pour une variation de charge unitaire (1 m).

La diffusivité, D ( $\left.\mathrm{m}^{2} / \mathrm{s}\right)$, est le rapport de la perméabilité sur le coefficient d'emmagasinement. Il exprime la rapidité du changement de la charge hydraulique à une variation des conditions aux limites. 


\subsection{8.}

\section{Porosité, degré de saturation et succion}

La porosité est déterminée à partir de la mesure de densité sèche et densité volumique de matrice rocheuse des grains sur des échantillons prélevés par sondages carottés.

L'évolution de la succion avec le degré de saturation est difficile à établir pour chaque ouvrage. Elle varie avec l'énergie de compactage : les plus gros pores sont réduits par le compactage. La relation entre la succion et la teneur en eau n'est pas unique : elle diffère en fonction du drainage. Enfin, les mesures sur sols grossiers et sols fissurés sont difficiles et très éloignées des mesures en laboratoire. Le choix de la courbe qui intervient dans le calcul est, pour ces raisons, un peu arbitraire. Les courbes utilisées sont rarement mesurées mais sont des courbes de référence issues de la littérature. Les expressions les plus utilisées sont :

- Van Genuchten

$$
\frac{\theta-\theta_{r}}{\theta_{s}-\theta_{r}}=\left\{\frac{1}{(1+\alpha \psi)^{n}}\right\}^{m}
$$

$\theta, \theta_{r^{\prime}} \theta_{s}$ : teneurs en eau volumiques courante, de rétention et de saturation ;

$\psi$ : succion totale en $\mathrm{m}$;

$\alpha, \mathrm{n}$ et m paramètres à fixer ;

- NS2D

$$
\frac{\theta}{\theta_{s}}=\frac{C}{C+\psi^{D}}
$$

$\theta, \theta_{\mathrm{s}}$ : teneurs en eau volumiques courante et de saturation;

$\psi$ : succion totale en $\mathrm{m}$;

$\mathrm{C}$ et $\mathrm{D}$ : paramètres à fixer.

La succion des sols compactés à l'énergie Proctor et à une teneur en eau proche de l’Optimum en laboratoire est donnée par Fleureau et al. :

$$
\begin{aligned}
& s=u_{\text {opt }} \cdot 10^{\left(\frac{w-w_{\text {opN }}}{d}\right)} \\
& u_{\text {opt }}=0,118\left(w_{L}\right)^{1,98} \\
& d=\frac{-\Delta W}{\Delta \log _{10}(s)} \\
& d=-0,54-0,03 w_{L}+3,3 \cdot 10^{-6}{W_{L}}^{2}
\end{aligned}
$$

$\mathrm{W}$ : teneur en eau en $\%$;

$\mathrm{W}_{\mathrm{L}}$ : limite de liquidité en $\%$;

$\mathrm{W}_{\text {OPN }}$ : teneur en eau de l'Optimum Proctor Normal en $\%$;

$\mathrm{u}_{\mathrm{opt}}:$ succion matricielle à l'OPN ;

$\mathrm{s}$ : succion matricielle en $\mathrm{kPa}$.

\section{A 19}

\section{Conductivité, perméabilité et perméabilité relative}

La perméabilité horizontale $\mathrm{k}_{\mathrm{x}}$ et la perméabilité verticale $\mathrm{k}_{\mathrm{y}}$ sont utilisées dans la pratique des calculs bidimensionnels. Pour minimiser le nombre de couches horizontales dans une fondation stratifiée, les perméabilités équivalentes horizontale et verticale sont calculées :
- perméabilité équivalente horizontale

$$
k_{e h} \sum_{i}^{N} e_{i}=\sum_{i}^{N} k_{i} e_{i}
$$

$\mathrm{k}_{\mathrm{eh}}$ : perméabilité équivalente horizontale ;

$\mathrm{k}_{\mathrm{i}}$ et $\mathrm{e}_{\mathrm{i}}$ : perméabilité et épaisseur de la couche $\mathrm{i}$;

- perméabilité équivalente verticale

$$
\frac{1}{k_{e V}} \sum_{i}^{N} e_{i}=\sum_{i}^{N} \frac{e_{i}}{k_{i}}
$$

$\mathrm{k}_{\mathrm{ev}}$ : perméabilité équivalente horizontale ;

$\mathrm{k}_{\mathrm{i}}$ et $\mathrm{e}_{\mathrm{i}}$ : perméabilité et épaisseur de la couche $\mathrm{i}$.

Quand les couches géologiques de la fondation sont toutes inclinées d'un angle $\theta$ par rapport à l'horizontale, compris entre $25^{\circ}$ et $65^{\circ}$, il est recommandé de définir le tenseur principal $\left(\mathrm{k}_{1}, \mathrm{k}_{2}\right)$ incliné comme le repère des couches géologiques d'un angle $\theta$ par rapport à l'horizontale.

Il convient de distinguer deux types de fondation, le sol et la roche. Le sol est poreux : il est traversé par une multitude de capillaires. La roche n'est pas poreuse quand elle n'est pas altérée, en revanche elle est souvent fissurée. Cette distinction explique des conditions aux limites différentes aux équations de la dynamique, et aboutit à une expression différente de la conductivité hydraulique :

- conductivité dans le sol

$$
k=n \frac{R^{2}}{8} \frac{\rho g}{\mu}
$$

n : porosité ;

$\mu$ : viscosité cinématique $10^{-3}$ Poiseuille à $20^{\circ}$;

$\mathrm{R}$ : rayon du capillaire moyen du sol ;

- conductivité dans la roche

$$
k=n \frac{e^{2} \rho g}{12 \mu}
$$

$\rho$ : masse volumique du fluide;

$g$ : accélération de la gravité ;

e : épaisseur de la fissure moyenne en $\mathrm{m}$.

La conductivité hydraulique des sols saturés est donc le produit de deux termes :

- un terme caractérisant le milieu, proportionnel à la porosité et au carré du rayon moyen des pores du sol ; - un terme caractéristique du fluide, rapport du poids volumique sur sa viscosité cinématique.

Ainsi un remblai dont la retenue a une température

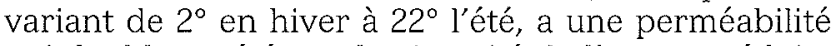
qui double en été, car la viscosité de l'eau est réduite de moitié.

\section{- Cas particulier des sols non saturés}

La perméabilité hydraulique des sols non saturés est inférieure à la perméabilité saturée. Le débit à travers un sol non saturé traverse une surface d'eau inférieure à celle du sol saturé et doit lutter contre des forces d'attraction surfacique d'autant plus grandes que le degré de saturation diminue. En conséquence le rapport des 
perméabilités du sol non saturé sur sol saturé, appelé perméabilité relative, diminue fortement avec le degré de saturation et l'augmentation de la pression capillaire. Cette relation entre perméabilité relative et degré de saturation est une donnée nécessaire au calcul du régime permanent. La perméabilité d'un sol pour un degré de saturation fixé est donc le produit de la perméabilité à saturation, décrite précédemment et de la perméabilité relative.

Parmi les expressions proposées dans la littérature, celles de NS2D et celles de Van-Genuchten Mualem sont rappelées ici :

- NS2D

$$
K_{r}(\psi)=\frac{a}{a+\psi^{b}}
$$

$\psi:$ succion $(\mathrm{m})$

$\mathrm{K}_{\mathrm{r}}$ : perméabilité relative ;

$a$ et $b$ paramètres de NS2D ;

$\alpha$, n et $m=1-1 / n$ paramètres de Mualem;

- Van Genuchten Mualem

$$
K r(\psi)=\frac{\left\{1-\frac{(\alpha \psi)^{n-1}}{\left[1+(\alpha \psi)^{n}\right]^{m}}\right\}^{2}}{\left[1+(\alpha \psi)^{n}\right]^{0,5}}
$$

L'intégration de la loi de la dynamique dans le domaine laminaire est à l'origine de l'expression de Hazen et de Vaughan de la perméabilité des sols granulaires sans argile (filtres et drains) et de Kozeny pour les sols avec argile.

Expression générale :

$$
\begin{aligned}
& k=k_{G E O} \frac{\rho g}{\mu}=k_{G E O} \frac{g}{v}=10^{7} \cdot k_{G E O} \\
& k_{G E O}=n \frac{d_{p}^{2}}{32}
\end{aligned}
$$

$v=$ viscosité dynamique ;

$v: 10^{-6} \mathrm{~m}^{2} / \mathrm{s}$ à $20^{\circ}$ pour l'eau ;

$\mathrm{k}_{\mathrm{GEO}}$ : perméabilité géométrique en $\mathrm{m}^{2}$.

Hazen

n : porosité ;

$\mathrm{k}$ : perméabilité en $\mathrm{m} / \mathrm{s}$;

$\mathrm{D}_{10}$ : diamètre du passant à $10 \%$ en $\mathrm{cm}$;

$\mathrm{d}_{\mathrm{p}}$ : diamètre moyen des pores en $\mathrm{m}$.

Vaughan

$$
\begin{aligned}
& d p=\frac{1}{100} \frac{D_{15}}{6} \\
& k=0,35 D_{15}^{2}
\end{aligned}
$$

$\mathrm{k}$ : perméabilité en $\mathrm{m} / \mathrm{s}$;

$\mathrm{D}_{15}$ : diamètre du passant à $15 \%$ en $\mathrm{cm}$.
Kozeny

$$
\begin{aligned}
& k=n C_{f} R_{H}^{2} \frac{\rho g}{\mu} \\
& R_{H} \cong \frac{e}{S_{p}} \\
& k=150 \frac{1}{f^{2}} \frac{1}{S_{p}^{2}} \frac{e^{3}}{1+e}
\end{aligned}
$$

$\mathrm{f}$ : coefficient de forme ;

$f=1,1$ pour un grain rond ;

$\mathrm{f}=1,5$ pour un grain anguleux ;

$$
\begin{aligned}
& \operatorname{div}(\operatorname{grad}(H))=\frac{C}{k} \frac{\partial H}{\partial t} \\
& \frac{\partial H}{\partial t}=D \cdot \operatorname{div}(\operatorname{grãd}(H))
\end{aligned}
$$

C : coefficient d'emmagasinement $\left(\mathrm{m}^{-1}\right)$;

$\mathrm{D}$ : diffusivité $\left(\mathrm{m}^{2} / \mathrm{s}\right)$

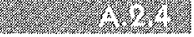 \\ Module de compressibilité et coefficient d'emmagasinement}

Dans les calculs transitoires de nappe captive, il est nécessaire de fixer le coefficient d'emmagasinement du terrain. Ce dernier peut être calculé par le logiciel en considérant le module de compressibilité comme le module isotrope à faible déformation (de l'ordre de 1E-4) provoquée par la variation du plan d'eau ou de la surface libre.

\section{1.}

\section{Génération de pressions interstitielles en fin de construction}

Il est pratique, comme le suggéra Bishop, d'utiliser le coefficient $r_{u^{\prime}}$ défini de la manière suivante :

$$
r_{\mathrm{u}}=\frac{u}{\gamma \cdot h}
$$

avec :

$\mathrm{u}$ : pression interstitielle;

$\gamma$ : poids volumique du sol au-dessus du point considéré ;

h : hauteur de la colonne de sol au-dessus du point considéré.

Le calcul est réalisé en imposant un coefficient constant par zone ou par matériau.

\section{A. 8}

\section{États limites à vérifier pour les digues comportant un rideau de palplanches}

Pour le cas des digues avec un écran de soutènement en palplanches métalliques, il convient de prendre en compte :

- les états limites de la digue du canal ;

- les états limites de l'écran de soutènement. 
Nous précisons ci-après les états limites à vérifier pour un rideau de palplanches métalliques. Cette annexe a été rédigée en s'inspirant de la norme NF EN 1993-5 (Eurocode 3, partie 5) et de ROSA 2000 (fascicule (c Rideaux de soutènement ) ou fascicule ( Exemples commentés de notes de calcul - rideau de soutènement ancré ı). Nous recommandons au lecteur de se reporter à ces deux ouvrages.

Pour le calcul des écrans de soutènement, la norme NF EN 1993-5 recommande de choisir l'approche de calcul 2 de l'Eurocode 7 qui consiste à appliquer les facteurs de sécurité aux actions ou à leurs effets et aux résistances. Dans certaines conditions, il est toutefois permis de justifier la stabilité générale du site selon l'approche de calcul 3, laquelle consiste à appliquer les facteurs partiels de sécurité aux actions ou à leurs effets et aux propriétés des matériaux.

\section{4}

\section{États limites ultimes}

Les états limites ultimes qui sont à vérifier pour un écran de soutènement en palplanches sont :

- la stabilité de l'écran vis-à-vis d'une rupture par défaut de butée (stabilité externe). Il s'agit de vérifier que la butée mobilisée est inférieure à la butée mobilisable (vérification de la fiche) ;

- la résistance de la structure de l'écran (stabilité interne). Il doit être vérifié que la résistance de la structure est suffisante pour supporter les efforts tranchants, moments fléchissants, efforts normaux, etc., qui sont appliqués. Dans le cas de rideaux de palplanches métalliques, la vérification de la résistance doit être effectuée selon la norme NF EN 1993-5;

- la portance de l'écran (stabilité externe). La vérification par le calcul de la capacité portante d'un écran n'est en principe à faire que dans le cas où l'écran supporte des charges verticales descendantes transmises par une structure portée et/ou par des tirants d'ancrage fortement inclinés. Dans ce cas, les charges verticales appliquées doivent rester inférieures aux charges admissibles que l'on calcule de façon analogue à celle des fondations profondes;

- la stabilité des tirants d'ancrage (stabilité interne). Il doit être vérifié la résistance de la structure des tirants et la résistance à l'arrachement de l'ancrage (longueur du scellement, résistance d'interaction terrain-ancrage dans le cas d'un ancrage scellé au terrain, ou butée des terres dans le cas d'un ancrage par contre-rideau) ;

- l'absence d'interaction entre l'écran et un ancrage (stabilité externe). Il s'agit de vérifier la stabilité du massif de terrain compris entre l'écran et l'ancrage et de s'assurer que les efforts d'ancrage appliqués au massif de sol sont exercés suffisamment loin de l'écran pour ne pas lui transmettre d'effort supplémentaire et générer davantage d'efforts sur la partie active de l'écran que le mécanisme de poussée traditionnel pris en compte lors des différentes étapes de son dimensionnement

- la stabilité du fond du lit (stabilité hydraulique). Il s'agit de vérifier la stabilité du fond du lit vis-à-vis d'une rupture par soulèvement hydraulique global du terrain (cette vérification vise les situations où un niveau (c étanche ) sous le fond du lit existe et est soumis à une pression hydraulique. Le soulèvement, provoqué par la poussée d'Archimède, se produit lorsque la pression de l'eau sous le fond du lit ( étanche » devient supérieure à la contrainte verticale moyenne due aux couches de terrain sus-jacente) ; par boulance (les particules de sol sont entraînées vers le haut et la rupture se produit par bouillonnement lorsque les forces d'écoulement ascendantes s'opposent au poids du sol et annulent la contrainte effective verticale) ; par érosion interne ; par érosion régressive ;

- la stabilité d'ensemble de l'écran (stabilité globale).

\section{A. 9}

\section{États limites de service}

Les états limites de service sont essentiellement associés aux déplacements et aux déformations (déplacement en tête du rideau, tassement du massif retenu, tassement et déplacement de la poutre de couronnement...). Habituellement, la justification de l'écran visà-vis des états limites de service consiste à vérifier :

- que les efforts supportés par l'écran restent admissibles pour la structure de l'écran (pour éviter par exemple une fissuration inacceptable, ou des problèmes de corrosion) ;

- et le cas échéant, que l'effort supporté par un tirant reste admissible pour sa structure et pour éviter un fluage important de l'ancrage.

Le déplacement de l'écran de soutènement est principalement lié :

- à sa réalisation (battage de palplanches, forage et injections des tirants, etc.) ;

- à ses déformations propres sous l'effet des sollicitations qui lui sont appliquées, donc à sa raideur et à celle de ses appuis ;

- aux déformations d'ensemble du massif, notamment dans le cas d'ancrages multiples ;

- à l'effet d'un rabattement, ou de la modification des niveaux d'eau (par exemple due à l'effet barrage d'un écran);

- au caractère gonflant ou évolutif des sols.

Pour la vérification du rideau de palplanches visà-vis de l'ensemble de ces états limites, il convient de tenir compte de l'influence des affouillements sur l'évolution du fond du lit (érosion des matériaux en pied de berges et diminution de la butée de pied).

\section{A 4}

\section{Détermination des sollicitations dues au vent, aux courants naturels et aux bateaux}

\section{1 . \\ Vagues dues au vent sur les retenues de barrages}

Lorsque le vent souffle sur un plan d'eau, il génère au bout d'une certaine durée des vagues qui peuvent se propager en direction du barrage. En supposant que le vent souffle en direction du barrage, les vagues formées déferlent sur le parement. En fonction de la différence d'altitude existant entre la cote du plan 
d'eau en situation de vent de projet et la crête, un certain pourcentage des vagues passe par-dessus la crête et ruisselle sur le parement aval. Une érosion plus ou moins importante de la crête et du parement aval en résulte. On doit donc positionner la crête à une altitude suffisante pour que le pourcentage de vagues qui l'atteignent soit très faible.

On considère classiquement deux situations de projet vis-à-vis du vent :

- un vent de période de retour 50 ans soufflant sur une retenue qui se trouve à la cote des $\mathrm{PHE}$; c'est cette situation qui s'avère généralement dimensionnante ;

- un vent de période de retour 1000 ans sur la retenue normale.

Pour chaque situation de projet vis-à-vis du vent, le calcul de la revanche s'effectue en deux étapes :

1) connaissant la vitesse du vent, on estime la hauteur des vagues; plutôt que la hauteur maximale des vagues, on s'intéresse à la hauteur significative (notée $\mathrm{H}_{\mathrm{s}}$ ), c'est-à-dire la hauteur moyenne du tiers supérieur de toutes les vagues du spectre;

2) on estime la hauteur de déferlement qui permet de fixer la revanche minimale nécessaire.

Pour le calcul de la hauteur des vagues, on propose d'utiliser la formule de Smith (1995), adaptée aux conditions d'eau profonde:

$$
H_{\mathrm{s}}=0,00048 \hat{U}_{\mathrm{a}} \sqrt{F}
$$

$\mathrm{H}_{\mathrm{s}}$ désigne la hauteur significative des vagues en $\mathrm{m}$; $\mathrm{F}$ est la longueur du fetch ${ }^{2}$ de la retenue en $\mathrm{m}$ (Fig. A.4.1) ;

$\hat{U}_{a}$ désigne la vitesse efficace ajustée du vent à $10 \mathrm{~m}$ de hauteur en $\mathrm{m} / \mathrm{s}$, soit $\hat{U}_{a}=U_{a}$. $\cos \theta$ avec $\theta$ l'angle entre les directions du vent et des vagues

$$
U_{a}=U \sqrt{(0,75+0,067 \times U)}
$$

avec $U$ vitesse moyenne sur une heure du vent à $10 \mathrm{~m}$ au-dessus de la surface de l'eau, pour la période de retour considérée. En l'absence d'information particulière sur la direction des vents, on prend $\hat{U}_{a}=U_{a}$.

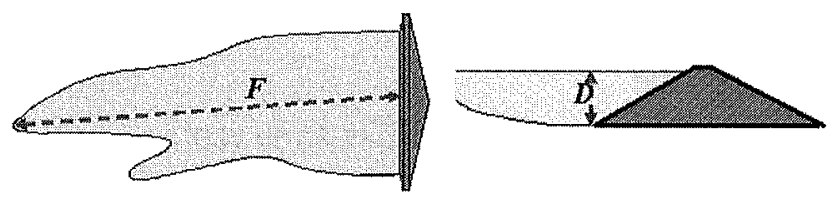

nc. A4. 9 Fetch et profondeur du plan d'eau.

(2) On appelle « fetch » la surface d'eau continue sur laquelle le vent souffle.
Dans le cas des vents supérieurs à $120 \mathrm{~km} / \mathrm{h}$, cette méthode donne de meilleurs résultats que celle de Bretschneider, dont les formules font également intervenir la profondeur $D$ du plan d'eau (Fig. A.4.1), et qui est proposée dans l'ouvrage [CFBR, 1997].

La durée minimale de vent $\left(t_{\min }\right)$ nécessaire à la formation des vagues peut être obtenue par application d'une autre formule de Smith (1995) :

$$
t_{\min }=\frac{27 F^{0,72}}{\hat{U}_{a}^{0,44}}
$$

La vitesse moyenne du vent sur une heure $\left(\mathrm{U}_{3600}\right)$ est liée à la vitesse $U_{t_{\min }}$ correspondant à $t_{\min }$ par la formule suivante (Shore Protection Manual, USACE, 1984)

$$
\frac{U_{t_{\min }}}{U_{3600}}=1,277+0,296 \cdot \tanh \left(0,9 \log \frac{45}{t_{\min }}\right)
$$

(formule valable pour $t_{\min }<3600 \mathrm{~s}$ ).

Un calcul itératif permet de déterminer $U_{a}$ correspondant à la donnée du vent mesurée au sol ${ }^{3}$.

Les vitesses de vent données proviennent en général d'une station terrestre et doivent alors être corrigées du fait du passage du vent sur la surface très lisse du plan d'eau (majoration de 1,01 pour $\mathrm{F}=100 \mathrm{~m}, 1,06$ pour $F=500 \mathrm{~m}$ et 1,1 pour $\mathrm{F}=1000 \mathrm{~m}$, selon Saville (1962)).

Le tableau A.4.I ci-après donne les valeurs de la hauteur significative des vagues pour des cas courants (profondeur de l'eau $D$ et longueur du fetch $F$ en mètres), calculée par la méthode de Smith. Pour $D=5 \mathrm{~m}$, les valeurs sont un peu différentes, car elles résultent de l'application de formules valables en eau non profonde, dues à Bretschneider et Reid [USACE, 1984]. Elles conduisent à des valeurs légèrement inférieures, d'au plus $6 \%$ dans les exemples du tableau.

La hauteur de vague de projet permettant de calculer la revanche minimale est ensuite estimée par rapport à la vague significative. Pour les retenues dont le parement aval est enherbé ou protégé, on peut tolérer que $5 \%$ des vagues atteignent la crête. Dans cette hypothèse, il faut prendre comme hauteur de vague de projet (ICE, 1996) : $\mathrm{H}_{\mathrm{d}}=1,25 \mathrm{H}_{\mathrm{s}}$.

Ayant obtenu la hauteur de la vague de projet, on calcule la hauteur de déferlement (run-up en anglais), notée $R$, qui est la différence de hauteur verticale entre le niveau maximal atteint par le déferlement de la vague sur le talus et le niveau du plan d'eau.

Le premier cas est celui d'un parement amont lisse. Le déferlement relatif de la vague $\left(\mathrm{R} / \mathrm{H}_{\mathrm{d}}\right)$ est donné par le tableau A.4.II dû à Saville (1962) en fonction de la pente du talus et de $\mathrm{H}_{\mathrm{d}} / \mathrm{L}$. ; L, longueur d'onde des

\footnotetext{
${ }^{(3)} \mathrm{La} 2^{\mathrm{e}}$ itération consiste à recalculer $U$ correspondant au $t_{\min }$ précédemment calculé par la formule (4), puis $U_{a}$ par (2), puis $t_{\text {min }}$ correspondant à ce nouveau $U_{a}$ par (3). Et ainsi de suite. On s'arrête lorsque $\mathrm{t}_{\min }$ est stabilisé et 3 ou 4 itérations suffisent. Les tableaux fournis dispenseront en général de faire ces calculs.
} 
vagues, est donnée par : $L=1,56 T^{2}$, formule valable si $\mathrm{D}>\mathrm{L} / 2$. $T$ est la période du pic spectral des vagues et peut être estimée, par une formule due à Smith [4] :

$$
\mathrm{T}=0,0716 \mathrm{~F}^{0,28} / \hat{\mathrm{U}}_{\mathrm{a}} .
$$

Ces résultats conduisent aux valeurs de revanche du tableau A.4.III.

Le second cas, est celui d'un parement amont rugueux. Les vagues déferlent alors moins haut. On propose, selon les publications de l'Institution of Civil Engineers (1996) et le South African Committee on Large Dams (1990), de prendre $60 \%$ de la revanche nécessaire pour un parement amont lisse. D'où le tableau A.4.IV construit à partir du tableau A.4.III.

Quelle que soit la rugosité du parement amont, pour une pente de $1 / 2,5$, les valeurs de déferlement obtenues pour une pente de $1 / 3$ sont à multiplier par 1,2. Pour une pente de $1 / 2$, elles sont à multiplier par 1,4 .
La revanche doit être calculée dans les deux combinaisons rares suivantes : à partir de la cote des PHE avec un vent de période de retour 50 ans et aussi à partir de la RN avec un vent de période de retour 1000 ans. En l'absence de données pour des périodes de retour élevées, on peut admettre que la vitesse du vent pour une période de retour 1000 ans vaut 1,2 fois celle pour 50 ans (ROSA, 2000). Par exemple si la vitesse de vent cinquantennale vaut $150 \mathrm{~km} / \mathrm{h}$ (colonne centrale des tableaux), la vitesse millénale vaut $180 \mathrm{~km} / \mathrm{h}$ (colonnes de droite).

Nous constatons que la revanche nécessaire pour les vagues varie dans une large gamme en fonction des conditions de vent, de la longueur de la retenue, de la pente du talus amont et de sa rugosité. Un calcul est donc indispensable à chaque fois. Le cas d'un parement lisse est nettement plus défavorable.

\begin{tabular}{|c|c|c|c|c|c|c|c|}
\hline 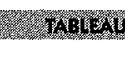 & $\begin{array}{l}\text { eur si } \\
\text { lue (F) }\end{array}$ & $\begin{array}{l}\text { des } \\
\text { rofor }\end{array}$ & en fo & la vite & at au & la lon & etch de la \\
\hline $\mathrm{D}(\mathrm{m}) \downarrow$ & $F(m)$ & 100 & 500 & 100 & 500 & 100 & 500 \\
\hline 5 & & 0,27 & 0,59 & 0,49 & 0,98 & 0,64 & 1,26 \\
\hline 10 & & 0,28 & 0,61 & 0,49 & 1,02 & 0,64 & 1,34 \\
\hline 15 & & 0,28 & 0,61 & 0,49 & 1,02 & 0,64 & 1,34 \\
\hline
\end{tabular}

rastexu Adid Valeurs du déferlement relatif $\mathrm{R} / \mathrm{H}_{\mathrm{d}}$ sur un parement lisse.

\begin{tabular}{llll}
\hline Pente du talus amont $\rightarrow$ & $1 / 3$ & $1 / 2,5$ & $1 / 2$ \\
$\mathrm{H}_{\mathrm{d}} / \mathrm{L}=0,1$ & 1,15 & 1,4 & 1,9 \\
$\mathrm{H}_{\mathrm{d}} / \mathrm{L}=0,08$ & 1,37 & 1,64 & 2,0 \\
$\mathrm{H}_{\mathrm{d}} / \mathrm{L}=0,07$ & 1,49 & 1,73 & 2,0 \\
\hline
\end{tabular}

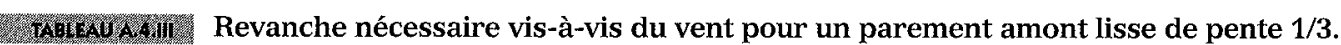

\begin{tabular}{|c|c|c|c|c|c|c|c|}
\hline $\mathrm{D} \downarrow$ & $F \rightarrow$ & $100 \mathrm{~m}$ & $500 \mathrm{~m}$ & $100 \mathrm{~m}$ & $500 \mathrm{~m}$ & $100 \mathrm{~m}$ & $500 \mathrm{~m}$ \\
\hline $5 \mathrm{~m}$ & & 0,53 & 1,13 & 0,71 & 1,48 & 0,92 & 1,85 \\
\hline $10 \mathrm{~m}$ & & 0,53 & 1,19 & 0,71 & 1,61 & 0,92 & 2,06 \\
\hline $15 \mathrm{~m}$ & & 0,53 & 1,19 & 0,71 & 1,63 & 0,92 & 2,06 \\
\hline
\end{tabular}

1AmExU A47N Revanche nécessaire vis-à-vis du vent pour un parement amont rugueux de pente 1/3.

\begin{tabular}{|c|c|c|c|c|c|c|c|}
\hline $\mathrm{D} \downarrow$ & $\mathrm{F} \rightarrow$ & $100 \mathrm{~m}$ & $500 \mathrm{~m}$ & $100 \mathrm{~m}$ & $500 \mathrm{~m}$ & $100 \mathrm{~m}$ & $500 \mathrm{~m}$ \\
\hline $5 \mathrm{~m}$ & & 0,32 & 0,68 & 0,43 & 0,89 & 0,55 & 1,11 \\
\hline $10 \mathrm{~m}$ & & 0,32 & 0,72 & 0,43 & 0,96 & 0,55 & 1,23 \\
\hline $15 \mathrm{~m}$ & & 0,32 & 0,72 & 0,43 & 0,98 & 0,55 & 1,23 \\
\hline
\end{tabular}




\section{4.}

\section{Courants naturels}

Le cas qui engendre les risques d'affouillements maximum intervient lors des crues, et en particulier dans les courbes. La zone la plus affectée est d'abord le pied de la berge.

Les modèles d'écoulement 1D sont généralement efficaces dans le cas des courants moyens au sein de systèmes de courants bien définis caractérisés par des concentrations prononcées de l'écoulement. Toutefois, dans certains cas, il est nécessaire d'avoir recours à des modèles d'écoulement 2D ou 3D. Il faut choisir avec soin le type de schématisation et le mode de calcul afin d'obtenir des résultats réalistes (référence, CETMEF 455).

Il existe trois principaux types de modèles permettant de calculer les niveaux d'eau et les vitesses du courant en rivière :

- les modèles courantologiques 2D, qui donnent un courant moyenné sur la profondeur en un point donné ;

- les modèles courantologiques 1D, qui donnent un courant moyenné sur l'intégralité de la section transversale ;

- les modèles hybrides, qui couplent un modèle de chenal en $1 \mathrm{D}$ à un modèle de réservoir de stockage situé dans la plaine inondable et calculent les échanges d'eau entre le lit mineur et la plaine inondable, ou dans la plaine inondable elle-même.

La principale précaution est sans aucun doute de veiller à l'exactitude des conditions aux limites sur lesquelles repose le modèle (débits, niveaux d'eau). Les variations spatiales (gradients), notamment, sont critiques dans le cas des modèles visant à résoudre des équations relatives à la morphologie. En règle générale, les conditions aux limites ayant trait aux niveaux d'eau et/ou aux débits doivent être positionnées suffisamment loin de l'ouvrage pour que les perturbations numériques éventuelles s'atténuent avant d'atteindre la zone étudiée.
Les modèles d'écoulement utilisés sont choisis en fonction de l'objet de l'étude et de la complexité des situations analysées (tableau ci-après).

En vue du dimensionnement des protections, il est d'usage de considérer deux situations :

- berges en alignement droit ;

- berges en courbes.

\section{- Berges en alignement droit}

La vitesse de l'écoulement moyennée sur la section, $U(\mathrm{~m} / \mathrm{s})$, est évaluée au moyen de la formule de Manning-Strickler :

$$
\mathrm{U}=\left(\mathrm{R}^{2 / 3} \mathrm{i}^{1 / 2}\right) / \mathrm{n}
$$

où :

$\mathrm{R}=$ rayon hydraulique (rapport de la surface mouillée au périmètre mouillé) $(\mathrm{m})$;

$i=$ pente de la ligne d'énergie ou pente de la ligne d'eau $(-)$;

$\mathrm{n}=$ coefficient de rugosité de Manning (-).

Le coefficient de rugosité de Manning $n$ tient compte du fait que la rugosité des berges et du lit entraîne des pertes de charge par frottement. Celles-ci augmentent avec la rugosité. Cette dernière dépend principalement de la nature des matériaux qui constituent le lit et de la végétation.

\section{- Berges en courbes}

La vitesse locale est déterminée comme suit en fonction de la vitesse moyenne d'écoulement :

$$
\mathrm{V}_{\text {locale }}=\mathrm{K} \cdot \mathrm{V}_{\text {moyen }}=\mathrm{K} \cdot(\mathrm{Q} / \mathrm{S})
$$

où :

$\mathrm{V}_{\text {locale }}=$ vitesse locale maximale au droit de la berge $[\mathrm{m} / \mathrm{s}]$;

$\mathrm{K}=$ coefficient de survitesse dans la coube $(-)$;

$\mathrm{Q}=$ Débit liquide $\left(\mathrm{m}^{3} / \mathrm{s}\right)$;

$\mathrm{S}=$ section mouillée $\left(\mathrm{m}^{2}\right)$.

\begin{tabular}{|c|c|c|}
\hline $\begin{array}{l}\text { Ligne de partage } \\
\text { des eaux }\end{array}$ & Concentration de l'eau dans les affluents & Modèles hyơrologiques \\
\hline 1 & Écoulement dans les vallées escarpées & $\begin{array}{l}\text { Modèles 1D } \\
\text { Modèles 1D torrentiels }\end{array}$ \\
\hline & Débordement du lit mineur & Modèle 1D \\
\hline & Zone de stockage des crues & $\begin{array}{l}\text { Modèles hybrides, couplant un modèle de chenal 1D } \\
\text { à un modèle de réservoir de stockage dans la plaine } \\
\text { inondable }\end{array}$ \\
\hline & $\begin{array}{l}\text { Écoulement dans les plaines inondables } \\
\text { où se trouvent des confluences, des affluents, } \\
\text { des ouvrages hydrauliques }\end{array}$ & Modèles 2D ou 3D \\
\hline$\downarrow$ & Écoulement en estuaire & Modèle 1D \\
\hline mer & Phénomène des marées, du vent et de la houle & $\begin{array}{l}\text { Modèles hybrides } \\
\text { Modèles 2D }\end{array}$ \\
\hline
\end{tabular}

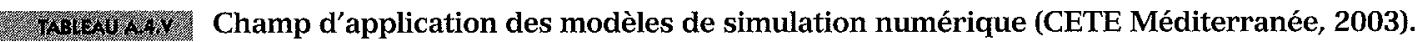


Il est à noter que ces résultats ont été obtenus pour les conditions suivantes :

$$
5 \leq \mathrm{B} / \mathrm{H} \leq 30 \text { et } \mathrm{V} /(\mathrm{g} \mathrm{H})^{0.5}<0,6
$$

Des essais systématiques ont été réalisés sur deux modèles physiques dont les formes ont été choisies pour représenter l'ensemble des formes géométriques des courbes naturelles rencontrées sur le Rhône. La formule mise au point, permettant une bonne concordance avec les limites de stabilité issues des essais sur modèle physique, a la même forme que celle de Ramette pour les parties en alignements.

La différence résulte de la vitesse du courant introduite dans la formule, qui est la vitesse locale au voisinage des enrochements calculée en multipliant la vitesse moyenne (ratio du débit liquide sur la section mouillée) par un coefficient multiplicateur dépendant uniquement (ainsi que l'ont montré les essais sur modèles) du rapport du rayon de la courbe $\mathrm{R}$ sur la largeur du lit en pied de berge B. Ce coefficient varie entre 1,0 et 1,4 suivant la position de la zone à protéger (entrée de courbe, milieu de courbe, intrados ou extrados) et la valeur du rapport R/B (Fig. A.4.2).

\section{(1)}

\section{Vagues dues au vent sur un canal}

Les vagues générées par les vents sollicitent la partie supérieure de la protection, de part et d'autre du plan d'eau. Cette zone peut donc varier en fonction $\mathrm{du}$ niveau d'eau considéré.

La taille des vagues dépend de l'intensité des vents et de leur durée, de la longueur du fetch $\mathrm{L}(\mathrm{m})$, et de la profondeur de l'eau h (m). On a établi le classement suivant :

- eau profonde : $\mathrm{h} / \mathrm{L}>0,25$;

- eau de profondeur intermédiaire : $0,05<\mathrm{h} / \mathrm{L}<0,25$;

- eau peu profonde $: \mathrm{h} / \mathrm{L}<0,05$.

Les vagues dues au vent peuvent être dimensionnantes dans les secteurs les plus larges de la rivière, à cause notamment de l'importance du fetch.

Dans le cas des eaux profondes, les paramètres caractéristiques des vagues peuvent être calculés par la méthode de l'US Army corps of engineers disponible sous forme d'abaque.
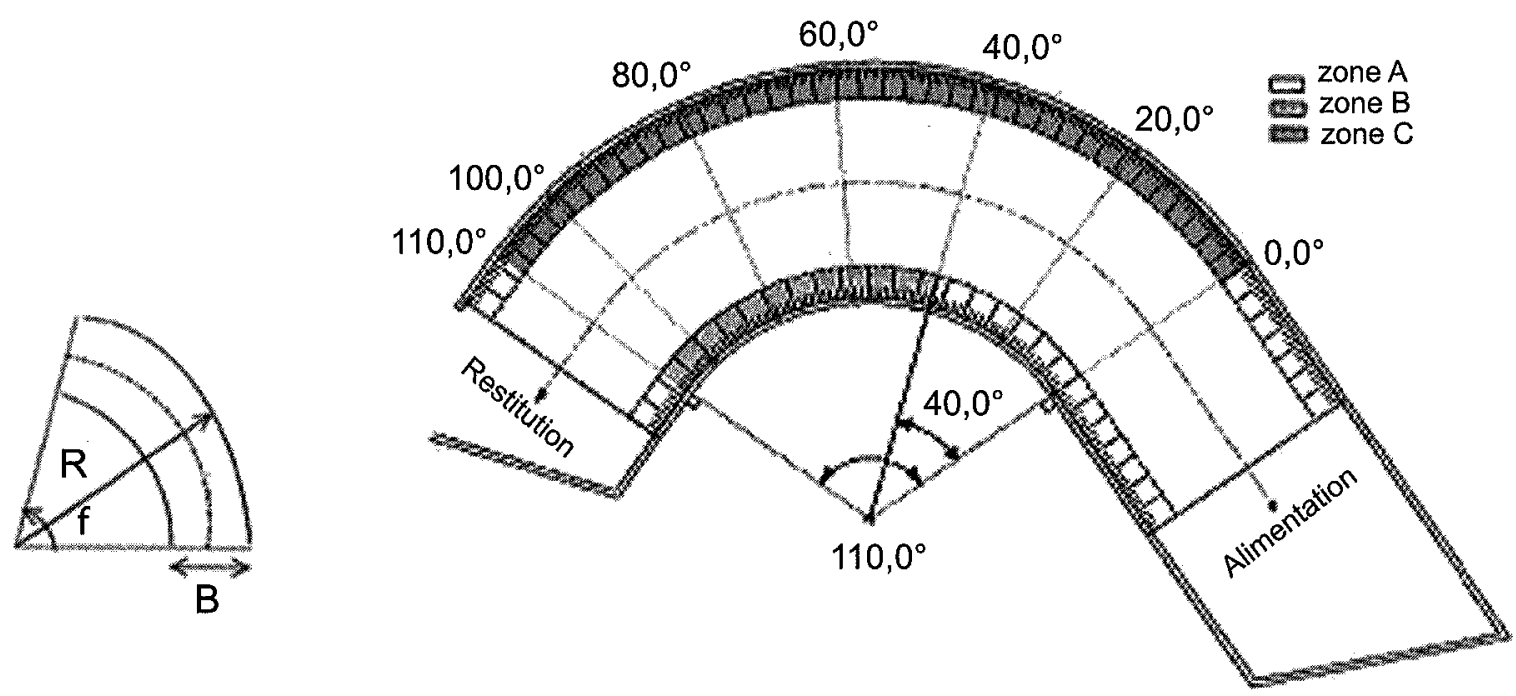

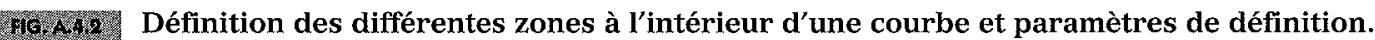

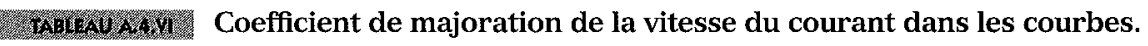

\begin{tabular}{|c|c|c|c|}
\hline $\begin{array}{c}\text { Intrados } \\
\text { (entrée de courbe) }\end{array}$ & ZONE A & $\begin{array}{c}2 \leq \mathrm{R} / \mathrm{B} \leq 4 \\
4 \leq \mathrm{R} / \mathrm{B} \leq 6 \\
6<\mathrm{R} / \mathrm{B}\end{array}$ & $\begin{array}{l}1,4 \\
1,2 \\
1,0\end{array}$ \\
\hline Intrados & ZONE B & $2 \leq R / B$ & 1,0 \\
\hline Extrados & ZONE C & $\begin{array}{c}2<\mathrm{R} / \mathrm{B}<8 \\
8 \leq \mathrm{R} / \mathrm{B}\end{array}$ & $\begin{array}{l}1,2 \\
1,0\end{array}$ \\
\hline
\end{tabular}




\section{Sollicitations liées à la navigation}

La navigation engendre des sollicitations multiples telles qu'illustrées dans la figure A.4.3. Leur importance et intensité dépendent du poids de l'embarcation et de sa vitesse, mais aussi bien entendu de sa distance au rivage.

Le batillage sollicite la partie supérieure de la protection. Il engendre deux sollicitations au niveau de la berge :

- déplacement en masse du liquide du fait de l'effet piston du bateau, qui se traduit principalement par la formation de l'onde de poupe et du courant de retour ; - formation d'ondes se propageant du bateau vers la berge (ondes secondaires).

Cette dualité se traduit également par des formules de dimensionnement différentes. Le diagramme suivant indique la démarche à adopter pour la détermination de ces différentes sollicitations.

Note. Les pointillés représentent les crêtes d'interférence des ondes secondaires, qui se propagent à $35^{\circ}$ par rapport au sens de la navigation.
Nous recommandons de suivre la démarche proposée dans le guide Enrochements (2009) pour la détermination des ondes de poupe, courants de retour et ondes secondaires.

\section{4.:5}

\section{Ondes de poupes et courants de retour}

Les abaissements moyens du plan d'eau et le courant moyen de retour sont calculés en suivant les étapes suivantes:

- Étape 1. Détermination de la section transversale immergée $A_{m}$

On applique l'équation suivante : $A_{m}=C_{m} \cdot B_{S} \cdot T_{S}$ avec :

$C_{m}=$ coefficient du maître-couple (-), lié à la section du bateau ;

$B_{s}=$ largeur du bateau (m) ;

$T_{s}=$ tirant d'eau du bateau (m).

Les valeurs appropriées de $C_{m}$ sont:

$C_{m}=0,9$ à 1,0 pour les pousseurs et les bateaux navigants sur les eaux intérieures ;

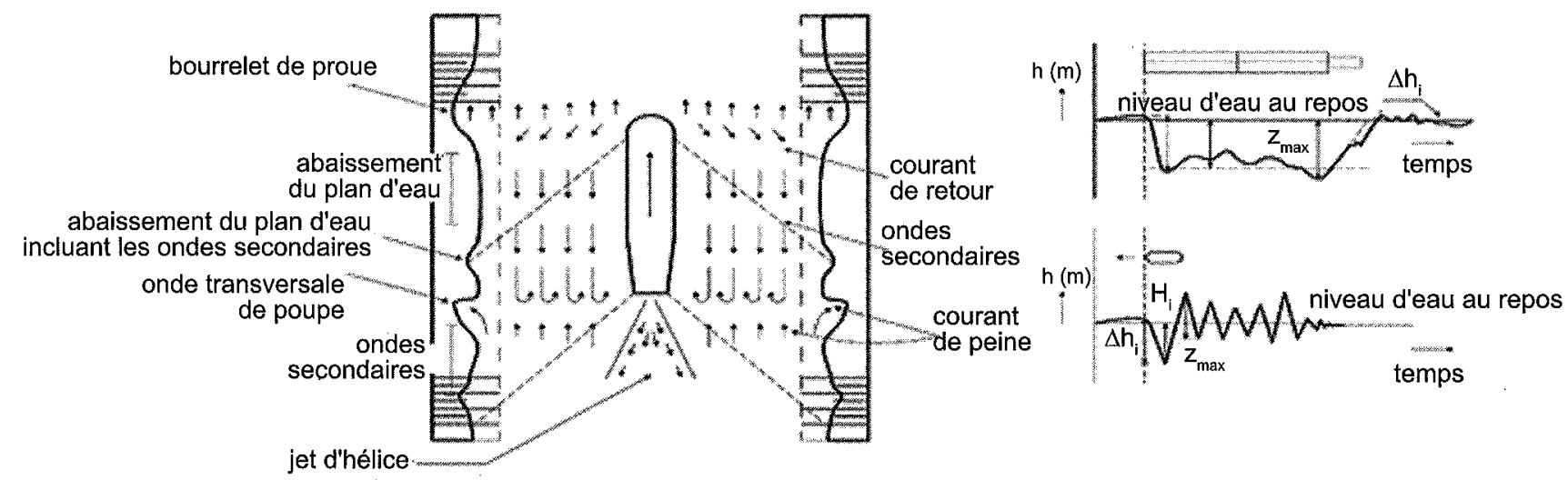

116. A.,3 Caractéristiques des mouvements induits par la navigation par rapport à la stabilité des berges (guide Enrochements, 2009).

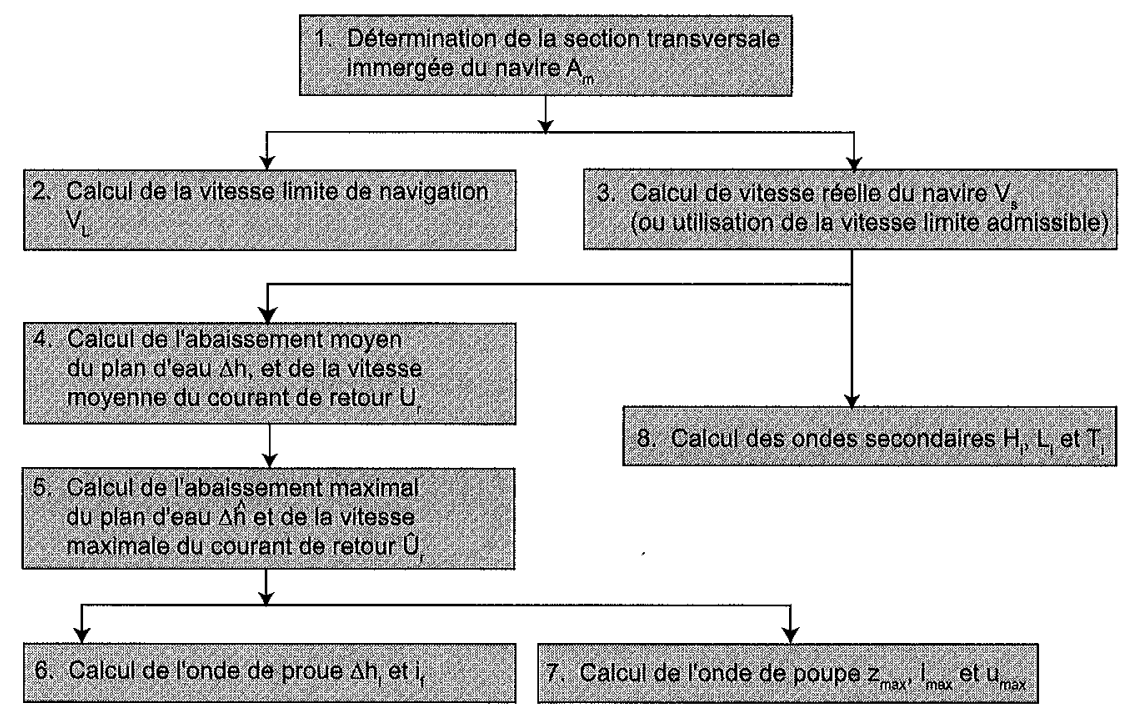

19. .444 Diagramme de calcul des mouvements de l'eau induits par la navigation (guide Enrochements, 2009). 
$C_{m}=0,9$ à 0,7 pour les navires de service, les remorqueurs et les navires de haute mer.

- Étape 2. Détermination de la vitesse limite de navigation $V_{L}$

La vitesse limite de navigation correspond à la valeur minimale entre les trois valeurs calculées à l'aide des équations suivantes:

1) $V_{L}=\sqrt{g h}$

2) $V_{L}=\sqrt{g L_{S} /(2 \pi)}$

3) $V_{L}=F_{L} \cdot \sqrt{\frac{g A_{C}}{b_{w}}}$

avec :

$\mathrm{F}_{\mathrm{L}}$ déterminé implicitement par la relation :

$$
F_{L}=\left[\frac{2}{3} \cdot\left(1-\frac{A_{m}}{A_{C}}+0,5 \cdot F_{L}^{2}\right)\right]^{3 / 2}
$$

$A_{c}=$ surface mouillée du chenal et $b_{w}=$ largeur au miroir du canal.

- Étape 3. Calcul de l'abaissement moyen du plan d'eau et du courant de retour

Il est calculé avec à partir de l'équation:

$$
\Delta h=\frac{V_{S}^{2}}{2 g} \cdot\left[\alpha_{S}\left(\frac{A_{C}}{A_{C}^{*}}\right)^{2}-1\right]
$$

avec :

$\alpha_{S}=1,40-0,4 \cdot \frac{V_{S}}{V_{L}}$

$A_{C}^{*}=b_{b} \cdot(h-\Delta h)+\cot \alpha \cdot(h-\Delta h)^{2}-A_{m} \quad$ section mouillée au droit du bateau

$A_{C}=b_{b} \cdot h+h^{2} \cdot \cot \alpha$ section mouillée normale; $b_{b}$ largeur au plafond du canal.

La vitesse moyenne du courant de retour, $U_{r}(\mathrm{~m} / \mathrm{s})$, est calculée à l'aide de l'équation :

$$
U_{r}=V_{S} \cdot\left(A_{C} / A_{C}^{*}-1\right)
$$

- Étape 4. Détermination de l'abaissement maximal du plan d'eau et hauteur maximale de l'onde d'étrave

L'abaissement maximal du plan d'eau $\Delta \hat{h}$ est déterminé à partir des équations suivantes :

- pour un bateau naviguant dans l'axe du canal, $\Delta \hat{h}$ vaut $\Delta \mathrm{h}$

- pour un bateau ne naviguant pas dans l'axe,

$$
\Delta \widehat{h}=\left(1+4 A_{w}^{*}\right) \cdot \Delta h \text { si } b_{w} / L_{S} \geq 1,5
$$

La hauteur maximale de l'onde d'étrave est alors déduite par la relation :

$$
\mathrm{z}_{\max }=1,5 \cdot \Delta \hat{\mathrm{h}}
$$

\section{6}

\section{Ondes secondaires}

La hauteur des vagues secondaires est évaluée au moyen de la formule de Havelock (guide Enrochements, 2009).
On peut considérer pour $\frac{V_{L}}{\sqrt{g h}}<0,8$ que :

$$
\begin{gathered}
H_{i}=1,2 \alpha_{i} \cdot h\left(y_{S} / h\right)^{-1 / 3} \cdot V_{S}^{4} /(g h)^{2} \\
L_{i}=4,2 \cdot \frac{V_{S}^{2}}{g}
\end{gathered}
$$

$\alpha_{i}$ : coefficient variant en fonction du type de bateau; les valeurs suivantes sont recommandées :

$\alpha_{i}=1$ dans le cas des remorqueurs, des bateaux de plaisance et des bateaux classiques chargés ;

$\alpha_{\mathrm{i}}=0,35$ dans le cas des bateaux classiques à vide;

$\alpha_{i}=1$ dans le cas des convois poussés à vide.

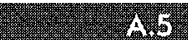

\section{Dimensionnement des protections de berge en enrochements (talus côté rivière ou canal)}

\section{4.}

\section{Méthodologie}

Elle consiste essentiellement à déterminer les éléments de dimensionnement suivants :

- dimensions maximales de la protection : profondeur, hauteur le long du talus et largeur de la banquette (dans le cas où elle est nécessaire) ;

- dimension moyenne des blocs ;

- introduction des corrections relatives à la pente, la courbure, la sinuosité et la densité des blocs;

- épaisseur de la protection;

- blocométrie (courbe granulométrique) ;

- couche filtre.

\section{W}

\section{Profondeur de la protection}

La profondeur de la protection dépend de la sollicitation considérée.

Pour la sollicitation liée à l'écoulement de l'eau, la protection couvre l'ensemble du parement soumis aux courants, dont l'intensité est en mesure de produire des affouillements.

Pour les vagues, la profondeur minimale, mesurée à partir du plan d'eau considéré, correspond à deux fois la hauteur de la vague significative $\mathrm{H}_{\mathrm{s}}$ ou est donnée par la formule de Hunt.

\section{3.}

\section{Dimension des blocs}

- Berges en alignement droit

La formule d'Isbash (équation suivante) permet de déterminer le diamètre nominal $\mathrm{D}$ de l'enrochement (diamètre nominal $=$ racine cubique du volume) : 
Formule d'Isbash :

$$
D=\frac{V^{2}}{m^{2} 2 g \alpha(\Delta)}
$$

$\mathrm{V}$ : vitesse du courant $(\mathrm{m} / \mathrm{s})$;

$m$ : coefficient d'Isbash dépendant de la position individuelle du bloc. Dans le cas des berges, il s'agit d'une couche continue et $m=1,4$;

$\alpha$ : coefficient de pente dépendant de la pente du talus :

$$
\alpha=\cos \phi \sqrt{1-\left(\frac{\sin \phi}{\sin \theta}\right)^{2}}
$$

$\phi$ : angle du talus avec l'horizontale ;

$\theta$ : angle au repos des matériaux $\left(\theta \geq 40^{\circ}\right)$;

$\Delta$ : densité apparente (déjaugée) des matériaux de protection $=\left(\rho_{\mathrm{s}}-\rho_{\mathrm{w}}\right) / \rho_{\mathrm{w}}=1,65$;

$\rho_{s}$ : masse volumique des matériaux;

$\rho_{\mathrm{w}}$ : masse volumique de l'eau.

La formule de Ramette (équation 6) permet d'obtenir le diamètre médian $\mathrm{D}_{50}$ des enrochements. Ce diamètre est ensuite multiplié par 1,3, coefficient partiel de sécurité (phase de crue).

$$
\frac{V^{2}}{g h^{\prime}}=\frac{A \varpi \lambda}{\varpi \frac{h^{\prime}}{D_{50}}\left[8,45+5,75 \lg \left(1,6 \frac{h^{\prime}}{D_{50}} \cos \phi\right)\right]^{-2}}
$$

$\mathrm{A}=$ coefficient indépendant des pentes des talus, établi expérimentalement $(A=0,02)$;

$\lambda=$ coefficient de Lane $=\cos \varphi \sqrt{1-\left(\frac{\tan \varphi}{\tan \theta}\right)^{2}} ;$

$\mathrm{V}=$ vitesse moyenne du courant au voisinage des enrochements $[\mathrm{m} / \mathrm{s}]$;

$\mathrm{h}^{\prime}=$ profondeur d'eau moyenne $[\mathrm{m}]$;

$\varpi=$ poids volumique de l'eau $=\rho_{\mathrm{w}} \cdot g=10 \mathrm{kN} / \mathrm{m}^{3}$;

$\phi=$ angle du talus de la berge par rapport à l'horizontale ;

$\boldsymbol{\theta}=$ angle d'équilibre des enrochements.

\section{- Berges en courbes}

Les formules d'Isbash et de Ramette indiquées plus haut demeurent valables. Cependant, les vitesses sont corrigées au moyen de coefficients de majoration allant jusqu'à 1,4.

Dans le cas des courbes, la formule d'Isbash prend la forme suivante :

$$
D=\frac{(\beta V)^{2}}{2 m^{2} g \alpha \Delta}
$$

Le coefficient de sinuosité $\beta$ rend compte de la sinuosité du lit, susceptible de générer des attaques de berge selon les angles d'incidence. Le degré de sinuosité est déterminé en fonction de l'angle A. Il est d'usage de considérer l'existence d'une sinuosité dans tous les cas où le courant est susceptible de changer de

\begin{tabular}{|c|c|c|c|}
\hline & Lit & Angle A & B \\
\hline & Rectiligne & 0 & 1 \\
\hline & Faible sinuosité & 30 & 1,5 \\
\hline & Forte sinuosité & 60 & 2,25 \\
\hline Angle de sinuosité & Angle droit & 90 & 4 \\
\hline
\end{tabular}
direction à cause d'un élément local : îlot, embâcle, etc. Le coefficient sert alors de coefficient partiel de sécurité dans ces cas.
Le coefficient $m$ d'Isbash constitue déjà un coefficient partiel de sécurité. C'est pour cela qu'il n'est pas d'usage de majorer le diamètre nominal.

- Vagues dues au vent

A partir de la hauteur significative des vagues $\left(\mathrm{H}_{\mathrm{s}}\right)$, il est possible de calculer le diamètre médian des enrochements à l'aide de la formule de Pilarczyck :

$$
D_{50} \geq \frac{H_{S} \sqrt{\xi}}{2,25 \delta_{m}}
$$

La hauteur de protection contre les vagues dues au vent est déduite de la formule de Hunt :

$$
\frac{R}{H_{S}}=0,7 \sqrt{2 \pi \xi}
$$

$\mathrm{R}=$ hauteur de déferlement des vagues ;

$\mathrm{H}_{\mathrm{s}}=$ hauteur des vagues ;

$\xi=$ nombre d'Iribarren.

\section{-}

\section{Résistance aux sollicitations liées à la navigation}

Le poids des enrochements de protection à prévoir correspond au maximum des poids calculés pour les deux types de sollicitations : ondes de poupe et ondes secondaires.

\section{- Ondes de poupes et courant de retour}

Le dimensionnement des enrochements vis-à-vis du courant de retour se fait comme pour le courant naturel en tenant compte de la combinaison du courant naturel et du courant de retour.

Le dimensionnement des enrochements vis-à-vis des ondes transversales de poupe est réalisé par l'application de la formule de Laborie :

$$
D_{50} \geq \frac{Z_{\max }}{K(\operatorname{cotan} \alpha)^{1 / 3} \Delta_{m}}
$$

$\mathrm{D}_{50}=$ diamètre moyen des enrochements de protection (m) ;

$\mathrm{Z}_{\max }=$ hauteur de l'onde transversale de poupe (m) ;

$\mathrm{K}=$ coefficient de stabilité $=1,5$;

$\alpha=$ angle de talus ;

$\Delta_{\mathrm{m}}=$ densité relative (déjaugée) des enrochements (prise égale à 1,6 dans le calcul).

\section{- Ondes secondaires}

Le dimensionnement des enrochements vis-à-vis des ondes secondaires est réalisé par l'application de la formule de Veyret et Pilaczyk. 


$$
D_{50} \geq \frac{H_{i} \cdot(\cos \beta)^{0.5}}{K \cdot \Delta_{m}}
$$

$D_{50}=$ diamètre moyen des enrochements de protection (m) ;

$\mathrm{H}_{\mathrm{i}}=$ hauteur des ondes secondaires (m) ;

$\mathrm{K}=$ coefficient de stabilité : 1,8;

$\beta=$ angle de propagation de la vague d'étrave (en général, égal à $55^{\circ}$ ) ;

$\Delta_{\mathrm{m}}=$ densité relative des enrochements (prise égale à 1,6 dans les calculs).

\section{A6s}

\section{Épaisseur de la protection}

L'épaisseur de la protection correspond à environ 2 fois le diamètre nominal $D_{50}$ des blocs, avec un minimum de $0,50 \mathrm{~m}$.

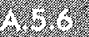

\section{Blocométrie}

La blocométrie doit être suffisamment étalée de sorte à faciliter la mise en place, mais limitée à un diamètre minimal afin de ne pas introduire de blocs de petite taille. Les rapports suivants (en fonction du diamètre nominal D) sont présentés à titre indicatif.

TABEesu sif Blocométrie.

\begin{tabular}{|c|c|c|}
\hline $0,90 \mathrm{D}$ & $1,12 \mathrm{D}$ & $1,40 \mathrm{D}$ \\
\hline
\end{tabular}

\section{(t)}

\section{Couche filtre}

En général, il est nécessaire de prévoir une couche filtre (f) dont le but principal est de prévenir l'érosion du matériau de la base à protéger (b) à travers la carapace d'enrochements.

Les critères de Terzaghi s'appliquent:

Critère de non-érosion de la base dans le filtre :

$$
\frac{D_{15 t}}{d_{85 b}}<4 \text { à } 5
$$

Critère de perméabilité suffisante :

$$
\frac{D_{15 f}}{d_{85 b}}>5
$$

( $f$ ) se réfère au matériau de la couche filtre ;

( $\mathrm{b}$ ) a au matériau de la base à protéger ;

$\mathrm{D}_{15 \mathrm{f}}$ et $\mathrm{d}_{85 \mathrm{~b}}$ représentent respectivement les diamètres obtenus sur la courbe granulométrique pour les passants à $15 \%$ et $85 \%$ en poids des particules.

\section{Dimension de la banquette de pied}

II n'existe pas de formules particulières pour dimensionner oes banquettes, mais une règle générale veut, qu'en cas d'affouillement, le volume de la banquette soit tel qu'il permette, en s'enfonçant dans la fosse daffouillement, de contre-buter constamment la couche d'enrochement.

Cette condition est obtenue si lon donne $\mathrm{a}$ la banquette un volume d'enrochement égal au volume de la couche d'enrochement multiplié par la profondeur de 1 affouillement.

Les banquettes seront généralement logées dans une tranchée de pied de section trapézotdale.

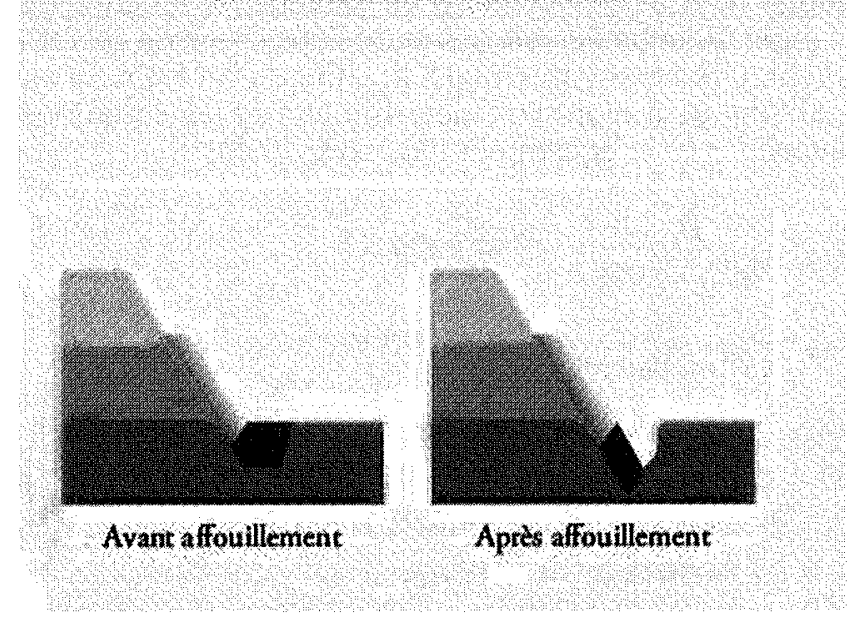

116.Ant. Protection d'une digue au moyen d'une carapace et d'une banquette (appelée aussi sabotoubêche parafouille)en enrochements (source CETE Méditerranée).

\section{Protections de talus côté rivière ou canal en matelas de gabions}

On peut se référer à la norme NF-P 94-325-2 et au guide Enrochements (principalement section 8.6.2), dont les principaux éléments sont repris dans cette annexe.

Il convient de prendre en compte les conditions de site (vitesse de courant, profondeur d'affouillement possible) et les données de l'ouvrage (type et état de l'ouvrage à protéger, niveau, type et état de la fondation de l'ouvrage) pour définir le niveau de pose, la géométrie et l'épaisseur de la protection superficielle parafouille. Pour les confortements de berges, le plus souvent, la protection superficielle est constituée par un radier rectangulaire réalisé à partir d'un assemblage de matelas gabions ou de gabions plats mis en place en pied, le long de l'ouvrage à protéger.

L'épaisseur de la protection superficielle doit être choisie en tenant compte des sollicitations dues à la vitesse du courant et de la hauteur de la section mouillée ou du tirant d'eau. Le tableau ci-après donne les règles approximatives concernant l'épaisseur des matelas de gabions, basées sur une fourchette de vites- 
ses du courant; les valeurs de vitesse critique et de vitesse limite sont destinées au dimensionnement préliminaire, en prenant pour hypothèse un revêtement horizontal (protection du lit) et des matelas de gabions à double diaphragme.

Pour la justification de la protection en matelas gabions vis-à-vis des courants, des calculs précis peuvent être effectués à partir de la contrainte de cisaillement exercée sur le revêtement (se référer notamment aux sections 8.6.2.3 et 5.2.1.3 du guide Enrochements). Lorsque le revêtement en gabions est soumis à l'action de vagues induites par la navigation ou par le vent, des essais réalisés par l'université de Delft (1983) ont permis de déterminer les hauteurs des vagues acceptables pour les revêtements en gabions, en fonction de l'angle du talus des berges et de l'épaisseur du revêtement. L'épaisseur minimale, $t_{\min }(\mathrm{m})$, du revêtement en gabions peut alors être déterminée par les équations suivantes (voir également Pilarczyk, 1998) :

$$
\begin{aligned}
t_{\min }=\frac{H}{2 \Delta\left(1-n_{v}\right) \cot \alpha} & \text { pour cot } \alpha \leq 3 \\
t_{\min } & =\frac{H}{4 \Delta\left(1-n_{v}\right)(\cot \alpha)^{1 / 3}} \quad \text { pour } \cot \alpha>3
\end{aligned}
$$

où :

$\mathrm{H}=$ hauteur de vague de dimensionnement $(\mathrm{m})$;

$\Delta=$ densité relative déjaugée de l'enrochement;

$\alpha=$ angle de talus de la berge ;

$\mathrm{n}_{\mathrm{v}}=$ porosité du matériau constitutif du revêtement. La valeur classique est de 0,35.

Le choix du niveau de pose et de l'épaisseur de la protection superficielle est souvent un compromis entre la performance de la protection vis-à-vis des sollicitations (vitesse de courant) et les contraintes liées à l'ouvrage. Un niveau de pose bas et une forte épaisseur de la protection assure en effet une meilleure efficacité de la protection parafouille, mais peuvent conduire à des travaux de re-profilage du terrain en place « à risque ) vis-à-vis de l'ouvrage à protéger et à une section mouillée ou un tirant d'eau insuffisant. Pour les ouvrages de protection superficielle de berge en gabionsmatelas ou en gabions plats soumis à un marnage, il convient que la cote inférieure du matelas protégeant la zone active du batillage soit située au moins $1,5 \mathrm{~m}$ sous le niveau des plus basses eaux navigables.

Enfin, le concepteur doit s'assurer que le sol sous la protection en gabions n'est pas érodé par la vitesse résiduelle de l'eau. En règle générale, il est nécessaire de placer un filtre géotextile sous les gabions pour éviter tout risque d'érosion.

\section{A.}

\section{Protection anti-érosion de talus côté rivière ou canal par techniques végétales}

Sur un cours d'eau navigable, on constate que des contraintes importantes sont rencontrées. Dans ce contexte, les techniques de protection $100 \%$ végétales ne peuvent être utilisées. La stabilisation désirée sera obtenue avec des techniques végétales dites mixtes constituées d'une structure compositie de matériaux inertes et vivants.

Si de l'emprise est disponible en berge et si une protection de berge ne s'impose pas, des zones :

- de non aedificandi écologique peuvent être préconisées aux abords du canal (anciennes zones industrielles) afin d'améliorer la qualité écologique des berges des canaux;

- des zones sans protections de berges peuvent être laissées vierges afin de favoriser des habitats intéressants (falaises à martins-pêcheurs...).

C'est l'interface terre-eau en milieu navigué qui est particulièrement soumise aux mouvements hydrauliques provoqués par les bateaux (ondes). La végétation, pour se maintenir, doit alors assurer une croissance moyenne supérieure à l'usure provoquée par le mouvement hydraulique et les autres facteurs de dégradations (animaux, fréquentation du public, intervention d'exploitation ou de gestion...).

Le plan d'eau étant maintenu tout au long. de l'année à un niveau quasi constant, près de la rive le sol est généralement gorgé d'eau et en anaérobiose plus ou moins poussée.

De nombreuses espèces rivulaires ne supportent pas ces conditions du milieu :

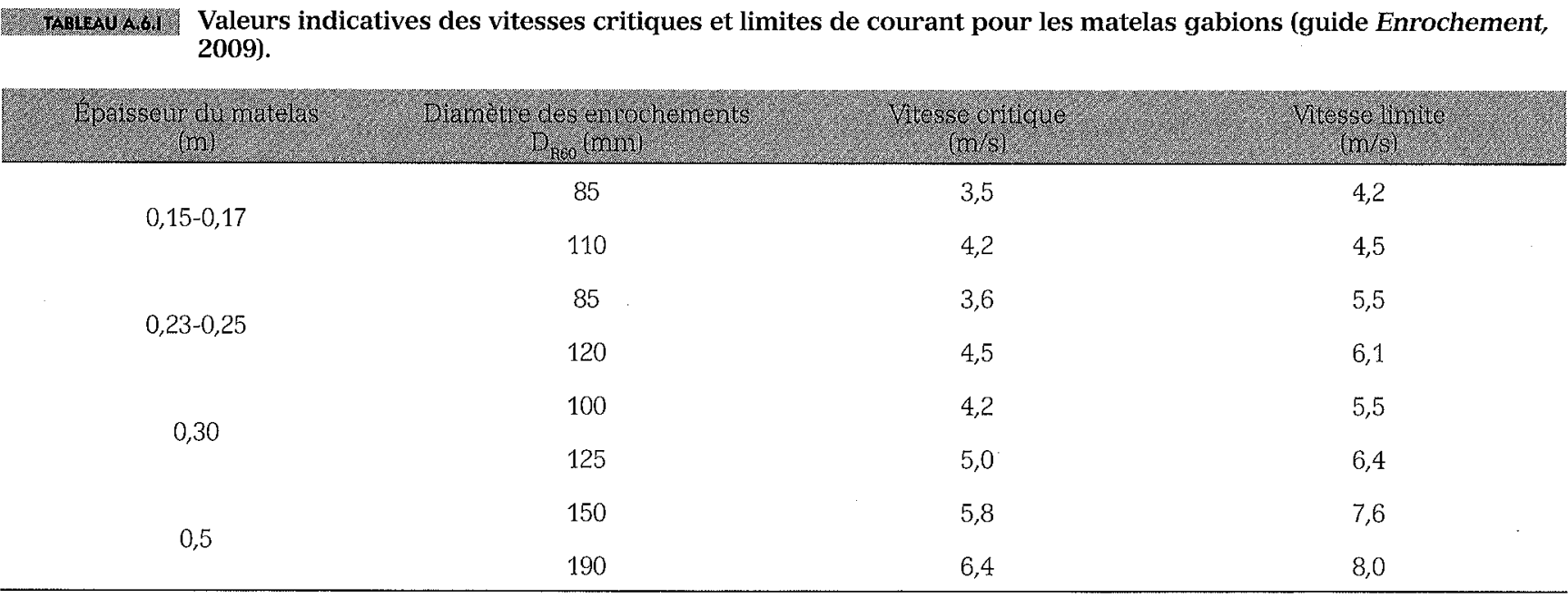


- hypoxie fréquente en zone racinaire ;

- agression mécanique pendant la saison de végétation.

Afin de dimensionner des protections de berge adaptées, il est essentiel de quantifier l'intensité des impacts liés aux ondes générées par les bateaux.

Leur adaptation aux différents niveaux de contraintes réside dans le dimensionnement des matériaux structurant la berge :

- augmentation de la blocométrie et élévation de l'arase du merlon pour le profil roselière ;

- utilisation de bois plus robustes et issu d'essences plus résistantes (chêne) ;

- hauteur de protection du pied de talus (gabions ou tunage végétalisé) ;

- protection du talus sous-fluvial (gabions matelas) ;

- arase pied de berge en enrochement plus haut (caisson bois).

Sur le haut de berge au-dessus de la limite d'influence de la vague générée par les bateaux, d'autres techniques purement végétales peuvent être mises en place : couches de branches, lit de plançons, etc. Ces techniques impliquent donc la présence obligatoire d'une butée de pied en enrochements par exemple. Une description rapide des techniques est présentée ci-dessous.

\section{Werlest}

\section{Haut fond végétalisé}

Ces merlons, avec une cote d'arase de plus de $30 \mathrm{~cm}$ au-dessus du niveau normal de navigation, permettent de protéger le pied de berge contre l'attaque des vagues afin que les végétaux mis en place en arrière puissent se développer sans stress. Ce profil est le plus intéressant écologiquement car il permet de créer un milieu de vie favorable pour les poissons (zone de croissance, de fraie) et pour les oiseaux en créant des zones de nidification. Ces merlons sont adaptables et dimensionnés selon les contraintes imposées par la navigation (fréquence, intensité...). Ils sont réalisés avec des enrochements dont la taille est proportionnelle aux contraintes.

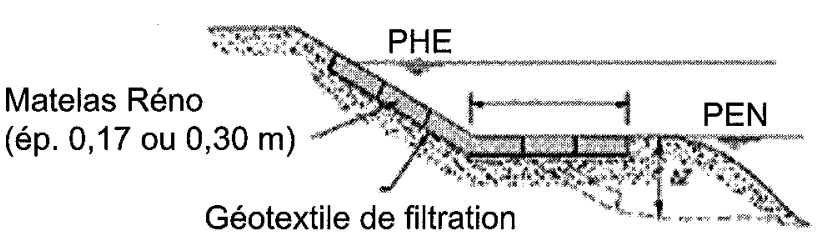

\section{A r}

\section{Matelas Réno végétalisés}

La technique de restauration par matelas Réno végétalisés est employée pour stabiliser les berges reprofilées, de pente $1 / 3$ à $2 / 3$. Ce sont des matelas grillagés d'environ $30 \mathrm{~cm}$ d'épaisseur remplis de matériaux pierreux. La géométrie dépend de la topographie de la berge (cf. figure ci-dessous).

De par son implantation, le matelas protège la berge sous-fluviale et terrestre. On recouvre et on fait pénétrer le matelas de terre végétale afin d’implanter de la végétation. La végétalisation se fait par plantations d'hélophytes et/ou bosquets de saules et enherbement.

Une variante composée d'une risberme est réalisable selon l'emprise.

\section{- \\ Gabions végétalisés}

La technique de restauration par gabions végétalisés est employée pour stabiliser les berges à forte pente (jusqu'à 2/1) ou sans emprise $(<1 \mathrm{~m})$ sur une hauteur pouvant atteindre 10 mètres (voir figure A.7.1).

Le gabion assure, de par sa structure en pierre, une stabilisation rigide immédiate et efficace contre les contraintes hydrauliques sur une hauteur de berge allant jusqu'à $1,5 \mathrm{~m}$. Les gabions sont remplis de matériaux terreux et de branches de saules couchées en rangs serrés.

L'utilisation d'un gabion terre-pierre permet l'implantation d'une végétation ligneuse dont les racines s'implantent rapidement dans la berge initiale. Cette technique apporte un aspect plus naturel à la berge par la mise en place de végétaux par rapport à un ouvrage de type ( gabions bruts ». Une bonne assise en enrochement est nécessaire afin de stabiliser l'ouvrage et pour sa pérennité.

\section{N}

\section{Enrochements végétalisés}

Cette technique est choisie afin d'implanter une végétation sur un profil composé d'enrochements

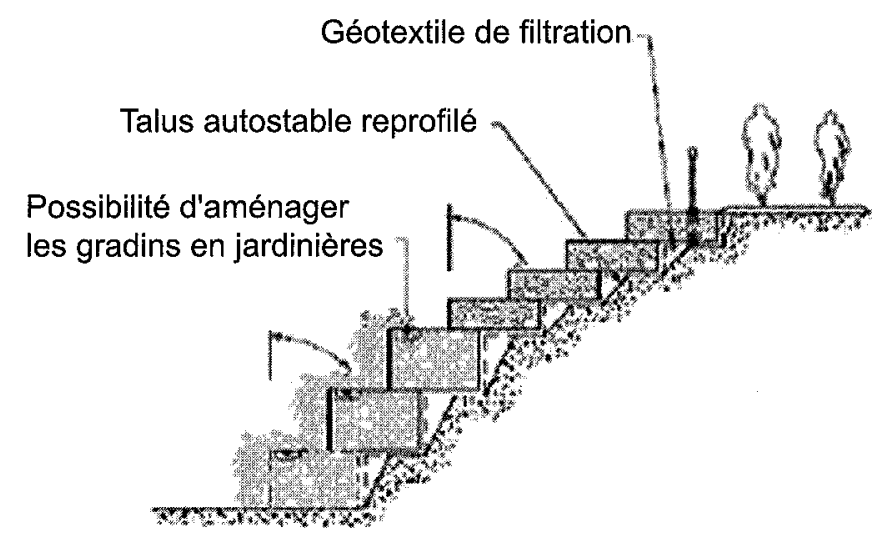


dénudés de toute végétation. Des plantations de boutures de saules et d'hélophytes sont réalisées dans les interstices des enrochements, préalablement percolés à l'aide d'un mélange de matériaux gravelo-limoneux. Les hélophytes sont positionnées en pied de berge à l'interface terre-eau soumise aux vagues générées par la navigation, les boutures, elles, sont employées sur l'ensemble du talus enroché. Cette végétalisation apporte ainsi une certaine diversité floristique et faunistique (entomofaune...).

\section{2. \\ Tunage végétalisé}

Le tunage de bois est utilisé pour protéger le pied de berge en réalisant une palissade de bois composée de pieux reliés par un platelage. Cette technique est généralement utilisée en zone de faible profondeur. Son pouvoir de soutènement est peu important : maximum 1 mètre.

Le tunage est considéré comme une technique végétale car il utilise du bois, matériau plus souple que les techniques dites dures utilisant les matériaux minéraux.

Afin de réaliser une intégration écologique de cet ouvrage, une végétalisation avec enherbement et plantation de plants d'hélophytes $(5 \mathrm{u} / \mathrm{ml})$ est réalisée en arrière.

Une alternative consiste à disposer des saules arbustifs sur le talus à la place des hélophytes.

Sa résistance aux sollicitations hydrauliques n'est pas précisément définie et dépend des matériaux utilisés, de la mise en place... Sa longévité est limitée par rapport aux autres solutions du génie végétal.

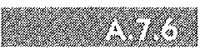 \\ Caissons végétalisés}

La technique de restauration par caisson végétalisé est employée lorsque l'emprise est faible en berge. Grâce à sa structure en bois, le caisson assure une stabilisation rigide immédiate et efficace contre les contraintes hydrauliques importantes sur une hauteur de berge allant jusqu'à 1,5 $\mathrm{m}$. Ce dernier est rempli de matériaux terreux et de branches de saules couchées en rangs serrés entre deux étages de rondins. Cette technique apporte un aspect plus naturel à la berge par la mise en place de végétaux par rapport à un ouvrage de type ( gabions ) ou murs. Une bonne assise en enrochement remontant au moins $30 \mathrm{~cm}$ au-dessus du NNN est nécessaire afin de stabiliser l'ouvrage et de protéger la berge des vagues provenant de la navigation.

\section{7.}

\section{Palplanches végétalisées}

Les palplanches végétalisées peuvent être utilisées pour éviter d'occuper une grande emprise en reprenant une profondeur d'eau importante tout en assurant une pente douce permettant de créer une plage d'hélophytes. Un talutage du haut de la berge par un apport de matériaux terreux compactés est nécessaire à l'implantation des hélophytes sur cette plage d'hélophytes de pente comprise entre 12:4 et 20:4 selon l'emprise disponible.

La résistance de cette technique aux sollicitations de la navigation est importante dans la mesure où elle permet, en fixant la cote de recépage du rideau de palplanches au niveau maximal de la houle calculée, de bien protéger la végétation disposée en arrière (hélophytes). 


\section{Bibliographie}

\section{Références générales}

CFGB - Petits barrages. Recommandations pour la conception, la réalisation et le suivi. Cemagref éditions, 176 p., 1997. http://www.barrages-cfbr.org/Info/ documentation/texte/pb2002/francais/ pb2002-s0.pdf

CIGB-ICOLD - Calcul statique des barrages en remblai, Bulletin, n 53, 1986.

CIRIA-CUR-CETMEF - The Rock Manual. The use of rock in hydraulic engineering, January 2007.

Fell R., et al. - Geotechnical engineering of dams, 2005.

Foster M., Fell R., Spannagle M. - The statistics of embankment dam failure and accidents. Can. Geotech. J. 37, 2000, p. 1000-1024.

Ministère de l'Équipement, du Transport et du Logement, CETMEF - ROSA (recommandations pour le calcul des états limites des ouvrages en site aquatique), 2000.

Norme NF-P 94-325-2, gabions.

Poulos S.J. - The strain-stress curves, 1971.

US Army Corps of Engineers - Design and Construction of Levees, EM 1110-2-1913, 2000.

US Army Corps of Engineers - Slope Stability, EM 1110-2-1902, 2003.

US Army Corps of Engineers - General Design and Construction Considerations for Earth and Rock-fill Dams, EM-1110$2-2300,2004$

\section{Analyse de risques}

Villemeur A. - Sûreté de fonctionnement des systèmes industriels, fiabilité, facteurs humains, informatisation. Paris, Éd. Eyrolles, 1988, 798 p.

Zwingelstein G. - La maintenance basée sur la fiabilité. Guide pratique d'application de la RCM, Éd. Hermes, 1996 $666 \mathrm{p}$

\section{Modèle géologique}

Antoine P., Barbier R. - Géologie des barrages de faible hauteur et de leur retenue. Annales de l'TTBTP, supplément au $n^{\circ} 312,1973$, p. 27-46.

Couturier B. - La géologie des barrages collinaires. Bulletin de l'AIGI, n 31,1985 , p. 51 à 57.

Couturier B. - Les études géologiques dans les projets de barrage. Thèse de doctorat d'État, université de Grenoble 1, 1987, $350 \mathrm{p}$.
Couturier B., Lautrin D., Brunel P. - Géologie et conception des petits barrages dans les molasses du Sud-Ouest de la France. Colloque technique sur les petits barrages, CFGB-AFEID, Bordeaux, 1993, p. 203-216.

Fauchard D., Mériaux P. - Méthodes géophysiques et géotechniques pour le diagnostic des diques de protection contre les crues. IREX-CEMAGREF, 2004 $124 \mathrm{p}$.

Fauchard D., Mériaux P. - Mise en œuvre des méthodes géophysiques à grand rendement pour le diagnostic des digues de protection contre les inondations. Ingénierie, $\mathrm{n}^{\circ}$ spécial, 2005, p. 83-90.

Gignoux M., Barbier R. - Géologie des barrages et des aménagements hydrauliques. Paris, Masson, 1955, 344 p.

ICOLD - Fondations de barrages. Bulletin $n^{\circ} 129,2005$.

Lautrin D. - Géologie des barrages et des retenues de petite dimension. CEMAGREF, ENITRTS, 1990, $144 \mathrm{p}$

Lérou J. - Géotechnique 1 (Cours). INSA Toulouse, 2005-2006.

Lugeon M. - Barrages et géologie. Paris, Dunod, 1932, $136 \mathrm{p}$.

US Army Corps of Engineers - Geotech nical investigations, EM-1110-1-1804 2001.

Wahlstrom E. - Dams, dam foundations and reservoir sites. Developments in geotechnical engineering. Elsevier scientific publishing company, 1974, $278 \mathrm{p}$.

Walters R. - Dam geology. Butterworth, $1962,336 p$

\section{Morphologie fluviale}

Degoutte G. - Diagnostic, aménagement et gestion des rivières : hydraulique et dynamique fluviales appliquées. Tec \& Doc éditions, 2006, $396 \mathrm{p}$

\section{Modèle géotechnique}

Barton N.R., Lien R., Lunde J. - Engineering classification of rock masses for the design of tunnel support. Rock Mech 6 (4), 1974, p. 189-239.

Bieniawski Z.T: - Geomechanics classification of rock masses and its application in tunnelling. Advances in Rock Mechanics 2, part A, Washington, DC National Academy of Sciences, 1974, p. 27-32.

Cedergren H.R. - Seepage, drainage and flow nets, 1989
Soares C. - Stability of compacted rockfill slopes. Géotechnique, vol. 34, $\mathrm{n}^{\circ}$ 1, 1984, p. $61-70$.

Hoek E. Brown E. T. - Underground Excavations in Rock. London, Instn Min Metall., 1980, $528 \mathrm{p}$.

Hoek E. - Strength of rock and rock masses. ISRM News Journal, 2 (2), 1994, p. 4-16.

Hoek E., Carranza-Torres C., Corkum B. The Hoek-Brown failure criterion. Proc. 5th North American Rock Mechanics Sym. and 17th Tunneling Assn of Canada conf. Toronto : NARMS-TAC, 2002 p. 267-271.

Mello V.F.B. - Reflections on design decisions of pratical significance to embank ment dams. Geotechnique, $n^{\circ} 3$, september, 1977, p. 281-355.

Philipponnat G., Hubert B. - Fondations et ouvrages en terre. Eyrolles, 2006, 550 p.

US Bureau of Reclamation - Earth manual Part 1 - 3rd Edition, 1998

\section{Modèle hydraulique}

US Army Corps of Engineers - Seepage Analysis and Control for Dams, EM 1110-2-1901, 1986.

\section{Sollicitations dues aux courants natu-} rels, au vent et aux bateaux

Institution of Civil Engineers - Floods and reservoir safety, third edition, Thomas Telford Publications, London, 1996 $64 \mathrm{p}$

Sancold - Interim guidelines on Freeboard for dams, Safety evaluation of dams, 1990.

Saville T., McClendon E., Cochran A. Freeboard Allowances for Waves in Inland Reservoirs. Journal of the Waterways and Harbours Division, ASCE, 1962.

Smith J. - Wind Wave Generation on Res tricted Fetches. Coastal Engineering Research Center, Waterways Experiment Station, Vicksburg, MS, 1991.

US Army Corps of Engineers - Coastal engineering manual, Waterways Experiment Station, Vicksburg, MS, 2003.

US Army Corps of Engineers - Shore protection manual, Waterways Experiment Station, Vicksburg, MS, 1984 